\title{
Moving Beyond Boron-based Substituents to Achieve Phosphorescence in Tellurophenes
}

William Torres Delgado, ${ }^{1}$ Christina A. Braun, ${ }^{1}$ Michael P. Boone, ${ }^{1}$ Olena Shynkaruk, ${ }^{1}$ Yanyu Qi, ${ }^{4}$ Robert McDonald, ${ }^{1}$ Michael J. Ferguson, ${ }^{1}$ Przemyslaw Data, ${ }^{2,3}$ Shawan K. C. Almeida, ${ }^{4}$ Inara de Aguiar, ${ }^{4}$ Gabriel L. C. de Souza, ${ }^{4}$ Alex Brown, ${ }^{1, *}$ Gang He, ${ }^{5, *}$ Eric Rivard ${ }^{1, *}$

${ }^{1}$ Department of Chemistry, University of Alberta, 11227 Saskatchewan Drive, Edmonton, Alberta, Canada, T6G 2G2

${ }^{2}$ Department of Physics, Durham University, Durham, UK, DH1 3LE

${ }^{3}$ Faculty of Chemistry, Silesian University of Technology, 44-100 Gliwice, Strzody 9, Poland

${ }^{4}$ Departamento de Química, Universidade Federal de Mato Grosso, Cuiabá, Mato Grosso, 78060-900, Brazil

${ }^{5}$ Center for Materials Chemistry, Frontier Institute of Science and Technology, Xi'an Jiaotong University, Xi'an, Shaanxi Province, 710054, P. R. China

\section{Contact Information of Corresponding Authors}

*E-mail: erivard@ualberta.ca, alex.brown@ualberta.ca, ganghe@mail.xjtu.edu.cn 


\section{TABLE OF CONTENTS:}

1. ${ }^{1} \mathrm{H},{ }^{13} \mathrm{C}\left\{{ }^{1} \mathrm{H}\right\},{ }^{11} \mathrm{~B}\left\{{ }^{1} \mathrm{H}\right\}$ and ${ }^{19} \mathrm{FNMR}$ Spectra

Figure S1. A) ${ }^{1} \mathrm{H}$ and B) ${ }^{13} \mathrm{C}\left\{{ }^{1} \mathrm{H}\right\}$ NMR Spectra of TPA-Te-6-TPA. S-8

Figure S2. A) $\left.{ }^{1} \mathrm{H}, \mathrm{B}\right){ }^{13} \mathrm{C}\left\{{ }^{1} \mathrm{H}\right\}$ and $\left.\mathrm{C}\right){ }^{11} \mathrm{~B}\left\{{ }^{1} \mathrm{H}\right\}$ NMR Spectra of Benzo-Te(BPin)TPA. S-9

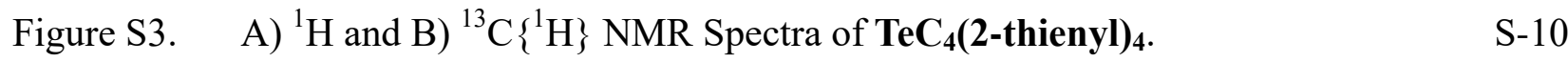

Figure S4. A) ${ }^{1} \mathrm{H}$ and B) ${ }^{13} \mathrm{C}\left\{{ }^{1} \mathrm{H}\right\}$ NMR Spectra of Naph-Te-6-Naph. S-11

Figure S5. A) ${ }^{1} \mathrm{H}$ and B) ${ }^{13} \mathrm{C}\left\{{ }^{1} \mathrm{H}\right\}$ NMR Spectra of Fl-Te-6-Fl. $\quad$ S-12

Figure S6. A) $\left.{ }^{1} \mathrm{H}, \mathrm{B}\right){ }^{13} \mathrm{C}\left\{{ }^{1} \mathrm{H}\right\}$ and C) ${ }^{19}$ F NMR Spectra of $\mathbf{A r} \mathbf{r}^{\mathbf{F}}$-Te-6-Ar ${ }^{\mathbf{F}}$. S-13

2. Absorption, Luminescence and Thermal Stability Data

Figure S7. UV-Vis absorption normalized of TPA-Te-6-TPA, TeC $_{4}$ (2-thienyl) 4 (in S-15 THF) and Benzo-Te(BPin)TPA (in cyclohexane).

Figure S8. Tauc plots of a) TPA-Te-6-TPA, b) Benzo-Te(BPin)TPA* and c) S-15 Te $_{4}$ (2-thienyl) $)_{4}$ in solution (THF and cyclohexane*). Inset: calculated optical band gaps.

Figure S9. UV-Vis absorption spectra of Naph-Te-6-Naph, Fl-Te-6-Fl, and S-16 $A \mathbf{r}^{\mathrm{F}}$-Te-6-Ar ${ }^{\mathrm{F}}$ in $\mathrm{THF}(30 \mu \mathrm{M})$ at room temperature under $\mathrm{N}_{2}$.

Figure S10. Tauc plots of a) Naph-Te-6-Naph b) Fl-Te-6-Fl and c) $\mathbf{A r}^{\mathrm{F}}$-Te-6-Ar ${ }^{\mathrm{F}}$ in S-16 THF; Inset: calculated optical band gaps.

Figure S11. Emission intensity of TPA-Te-6-TPA in different THF/water ratios (listed as S-17 $\%$ water content). Inset: aggregates under UV light $\left(\lambda_{\text {ex }}=365 \mathrm{~nm}\right)$. [TPA-Te-6-TPA] $=10^{-4} \mathrm{M}$.

Figure S12. Excitation (solid lines) and emission spectra (dashed lines) of S-17 TPA-Te-6-TPA in the solid state. $\lambda_{\mathrm{ex}}=365 \mathrm{~nm}, \lambda_{\mathrm{em}}=525 \mathrm{~nm}$.

Figure S13. UV-Vis absorption of Benzo-Te(BPin)TPA in THF, cyclohexane and DMF. S-18

Figure S14. Luminescence measurements $\left(\lambda_{\text {ex }}=350 \mathrm{~nm}\right)$ of $4 \mathrm{BT}$ in toluene, THF and S-18 methylcyclohexane $(10 \mu \mathrm{M})$ after degassing.

Figure S15. Luminescence measurements of a film of $\mathbf{4 B T e}$ under air and Ar, $\lambda_{\mathrm{ex}}=370$ S-19 $\mathrm{nm}$.

Figure S16. Solid state phosphorescence lifetimes of 4BTe under air, modeled with S-19 biexponential decays. 
Figure S17. Solid state phosphorescence lifetimes of 4BTe under Ar, modeled with S-20 biexponential decays.

Figure S18. Solid state phosphorescence of 4BTe $(1 \mathrm{wt} \%)$ in Zeonex ${ }^{\circledR}$ matrix at $300 \mathrm{~K} . \quad$ S-20 $\lambda_{\text {em }}=426 \mathrm{~nm} . \tau=1.06 \mathrm{~ns} . \lambda_{\text {em }}=520 \mathrm{~nm} . \tau=242.96 \mu \mathrm{s}$.

Figure S19. Solid state phosphorescence of $\mathbf{A r}^{\mathbf{F}}-\mathbf{T e}-\mathbf{6}-\mathbf{A r}^{\mathbf{F}}$ (32 wt\%) both in a PMMA S-21 matrix and in pure film at rt. $\lambda_{\mathrm{ex}}=360 \mathrm{~nm}$. $\lambda_{\mathrm{em}}=567$ and $574 \mathrm{~nm}$, respectively.

Figure S20. Solid state phosphorescence of Benzo-Te(BPin)TPA measured at $\mathrm{rt}, \lambda_{\mathrm{ex}}=\mathrm{S}-21$ $360 \mathrm{~nm}$.

Figure S21. Solid state phosphorescence of benzophenone measured at $\mathrm{rt}, \lambda_{\mathrm{ex}}=360 \mathrm{~nm} . \quad \mathrm{S}-22$

Figure S22. Solid state luminescence of benzophenone (BP) and Benzo-Te(BPin)TPA S-22 mixture (1:1) measured at rt, $\lambda_{\mathrm{ex}}=360 \mathrm{~nm}$.

Figure S23. Solid state emission of benzophenone (BP), Benzo-Te(BPin)TPA and their S-23 mixture (1:1) measured at rt, $\lambda_{\mathrm{ex}}=360 \mathrm{~nm}$.

Figure S24. Solid state emission of Benzo-Te(BPin)TPA, PMMA and their mixture S-23 measured at $\mathrm{rt}, \lambda_{\mathrm{ex}}=360 \mathrm{~nm}$.

Figure S25. Solid state luminescence of TPA-Te-6-TPA measured at rt, $\lambda_{\mathrm{ex}}=365 \mathrm{~nm} . \quad$ S-24

Figure S26. Solid state luminescence of a 1:1 mixture of TPA-Te-6-TPA and S-24 benzophenone (BP) measured at rt, $\lambda_{\mathrm{ex}}=365 \mathrm{~nm}$.

Figure S27 Solid state emission of TPA-Te-6-TPA and its 1:1 mixture with S-25 benzophenone (BP) measured at rt, $\lambda_{\mathrm{ex}}=365 \mathrm{~nm}$.

Figure S28 Solid-state emission of Naph-Te-6-Naph and its 1:1 mixture with S-25 benzophenone (BP) measured at $\mathrm{rt}, \lambda_{\mathrm{ex}}=395 \mathrm{~nm}$.

Figure S29. Luminescence in the solid state of A) $\mathbf{A r}^{\mathrm{F}}-\mathbf{T e}-\mathbf{6}-\mathbf{A} \mathbf{r}^{\mathrm{F}}$, B) Napt-Te-6-Naph S-26 and C) Fl-Te-6-Fl at room temperature when irradiated at $365 \mathrm{~nm}$.

Figure S30. TGA measurements of $\mathbf{T e C}_{4}$ (2-thienyl) ${ }_{4}$ and TPA-Te-6-TPA. S-26

$\begin{array}{llr}\text { Figure S31. TGA measurements of Benzo-Te(BPin)TPA. } & \text { S-27 }\end{array}$

\section{Crystallographic Data}

$\begin{array}{lll}\text { Figure S32. } & \text { Molecular structure of 4BSe with thermal ellipsoids presented at a } 30 \% & \text { S-28 }\end{array}$ probability level.

Table S1. X-Ray Crystallographic Data for Benzo-Te(BPin)TPA and S-29 $\mathrm{TeC}_{4}(2$-thienyl) 4 .

Table S2. X-Ray Crystallographic Data for Naph-Te-6-Naph and Ar ${ }^{\mathbf{F}}$-Te-6-Ar ${ }^{\mathbf{F}} . \quad$ S-30

$\begin{array}{lll}\text { Table S3. X-Ray Crystallographic Data for 4BSe. } & \text { S-31 }\end{array}$ 
4. Computational Results

4.1. Computational Methods

S-32

Table S4. The excitation energies (in eV), absorption wavelengths (nm), and oscillator strengths (f) of the lowest ten lying singlet and ten lying triplet states for TPA-Te-6-TPA at the TD-B3LYP/cc-pVTZ-(PP) level of theory at the gasphase $\mathrm{S}_{0}$ geometry (as determined at the B3LYP/cc-pVTZ-(PP) level of theory).

Figure S33. a) HOMO-2, b) HOMO, and c) LUMO for TPA-Te-6-TPA at the TDB3LYP/cc-pVTZ-(PP) level of theory at the gas-phase $\mathrm{S}_{0}$ geometry (as determined at the B3LYP/cc-pVTZ-(PP) level of theory). Excitation to $\mathrm{S}_{1}$ involves $\mathrm{HOMO} \rightarrow$ LUMO $(\mathrm{C}=0.69466)$. Excitation to $\mathrm{S}_{8}$ involves HOMO-2 $\rightarrow$ LUMO (C=0.69595, $3.5308 \mathrm{eV}, 351.15 \mathrm{~nm}, \mathrm{f}=0.0126)$. All orbitals illustrated with an isovalue $=0.02$.

Table S5. Comparison of selected bond lengths $(\AA)$ and angles $\left(^{\circ}\right)$ for BenzoTe(BPin)TPA determined from the experimental X-ray structure and those computed at the B3LYP/cc-pVTZ-(PP) level of theory in the gas-phase. Atom labels are provided in Figure 2 in the main text.

Table S6. $\quad \Lambda$-diagnostic for the $S_{1}$ and $S_{2}$ states for Benzo-Te(BPin)TPA at the TDB3LYP/cc-pVTZ-(PP) level of theory in the gas-phase as well as TD-CAMB3LYP/cc-pVTZ-(PP) and TD-M06-2X/cc-pVTZ-(PP) levels of theory in THF. All computations at the gas-phase $\mathrm{S}_{0}$ geometry (as determined at the B3LYP/cc-pVTZ-(PP) level of theory). Values less than 0.4 suggest chargetransfer or long-range character to the excitation.

Table S7. Excitation energies (in eV), absorption wavelengths (nm), and oscillator strengths (f) of the ten lowest-lying singlet and triplet states for Benzo-Te(BPin)TPA at the TD-B3LYP/cc-pVTZ-(PP), TD-CAMB3LYP/cc-pVTZ-(PP), and TD-M06-2X/cc-pVTZ-(PP) levels of theory in the gas-phase. All computations at the gas-phase $\mathrm{S}_{0}$ geometry (as determined at the B3LYP/cc-pVTZ-(PP) level of theory).

Table S8. Excitation energies (in eV), absorption wavelengths ( $\mathrm{nm}$ ), and oscillator strengths (f) of the ten lowest-lying singlet and triplet states for Benzo-Te(BPin)TPA at the TD-B3LYP/cc-pVTZ-(PP), TD-CAMB3LYP/cc-pVTZ-(PP), and TD-M06-2X/cc-pVTZ-(PP) levels of theory in THF (PCM). All computations at the gas-phase $\mathrm{S}_{0}$ geometry (as determined at the B3LYP/cc-pVTZ-(PP) level of theory).

Table S9. Nature of the lowest-lying singlet states $\left(\mathrm{S}_{1}, \mathrm{~S}_{2}\right.$, and $\left.\mathrm{S}_{3}\right)$ for BenzoTe(BPin)TPA at the TD-B3LYP/cc-pVTZ-(PP), TD-CAM-B3LYP/ccpVTZ-(PP), and TD-M06-2X/cc-pVTZ-(PP) levels of theory in the gasphase and THF (PCM). All computations at the gas-phase $\mathrm{S}_{0}$ geometry (as determined at the B3LYP/cc-pVTZ-(PP) level of theory). HOMO = Orbital 137 and LUMO = Orbital 138 . 
Figure S34. a) HOMO and b) LUMO corresponding to the lowest excitation to $\mathrm{S}_{1}$ for S-38 Benzo-Te(BPin)TPA at the TD-B3LYP/cc-pVTZ-(PP) level of theory at the gas-phase $\mathrm{S}_{0}$ geometry (as determined at the B3LYP/cc-pVTZ-(PP) level of theory).

Figure S35. a) HOMO-1, b) HOMO, c) LUMO, and d) LUMO+1 involved in the main contributions to the lowest two excitations for Benzo-Te(BPin)TPA at the TD-CAM-B3LYP/cc-pVTZ-(PP) level of theory at the gas-phase $\mathrm{S}_{0}$ geometry (as determined at the B3LYP/cc-pVTZ-(PP) level of theory). All orbitals illustrated with an isovalue $=0.02$.

Figure S36. a) HOMO-1, b) HOMO, c) LUMO, and d) LUMO+1 involved in the main contributions to the lowest two excitations for Benzo-Te(BPin)TPA at the TD-M06-2X/cc-pVTZ-(PP) level of theory at the gas-phase $\mathrm{S}_{0}$ geometry (as determined at the B3LYP/cc-pVTZ-(PP) level of theory). All orbitals illustrated with an isovalue $=0.02$.

Table S10. Comparison of selected bond lengths $(\AA)$ and angles $\left(^{\circ}\right)$ for $\mathbf{T e C}_{4}(\mathbf{2}-\mathrm{S}-41$ thienyl) $)_{4}$ determined from the experimental $\mathrm{X}$-ray structure and those computed at the B3LYP/cc-pVTZ-(PP) level of theory in the gas-phase. Atom labels are provided in Figure 4 in the main text.

Table S11. The excitation energies (in eV), absorption wavelengths (nm), and oscillator strengths (f) of the lowest ten lying singlet and triplet states for TeC $_{4}$ (2-thienyl) ${ }_{4}$ at the TD-B3LYP/cc-pVTZ-(PP) level of theory at the gasphase $\mathrm{S}_{0}$ geometry (as determined at the B3LYP/cc-pVTZ-(PP) level of theory).

Figure S37. a) HOMO-1, b) HOMO, and c) LUMO for $\mathbf{T e C}_{4}$ (2-thienyl) 4 at the TDB3LYP/cc-pVTZ-(PP) level of theory at the gas-phase $\mathrm{S}_{0}$ geometry (as determined at the B3LYP/cc-pVTZ-(PP) level of theory). Excitation to $\mathrm{S}_{1}$ involves $\mathrm{HOMO} \rightarrow$ LUMO $(\mathrm{C}=0.68437)$. Excitation to $\mathrm{S}_{3}$ involves HOMO-1 $\rightarrow$ LUMO+1 $(\mathrm{C}=0.65750,3.6113 \mathrm{eV}, 343.32 \mathrm{~nm}, \mathrm{f}=0.0521)$. All orbitals illustrated with an isovalue $=0.02$.

Table S12. Comparison of selected bond lengths $(\AA)$ and angles $\left(^{\circ}\right)$ for Naph-Te-6Naph determined from the experimental X-ray structure and those computed at the B3LYP/cc-pVDZ-(PP) level of theory in the gas-phase. Atom labels are provided in Figure 5 in the main text.

Table S13. The excitation energies (in eV), absorption wavelengths (nm), and oscillator strengths (f) of the lowest ten lying singlet and triplet states for Naph-Te-6-Naph at the TD-B3LYP/cc-pVTZ-(PP) level of theory at the gas-phase $\mathrm{S}_{0}$ geometry (as determined at the B3LYP/cc-pVDZ-(PP) level of theory).

Figure S38. a) HOMO-1, b) HOMO, c) LUMO, and d) LUMO+1, i.e., dominant orbitals S-44 involved in the low-lying excited singlet states, for Naph-Te-6-Naph at the TD-B3LYP/cc-pVTZ-(PP) level of theory at the gas-phase $\mathrm{S}_{0}$ geometry (as determined at the B3LYP/cc-pVTZ-(PP) level of theory). All orbitals illustrated with an isovalue $=0.02$. 
Table S14. The excitation energies (in eV), absorption wavelengths (nm), and oscillator strengths (f) of the lowest ten lying singlet and triplet states for Fl-Te-6-Fl at the TD-B3LYP/cc-pVTZ-(PP) level of theory at the gas-phase $\mathrm{S}_{0}$ geometry (as determined at the B3LYP/cc-pVTZ-(PP) level of theory).

Table S15. Comparison of selected bond lengths $(\AA)$ and angles $\left(^{\circ}\right)$ for $\mathbf{A r} \mathbf{r}^{\mathbf{F}}-\mathbf{T e}-\mathbf{6}-\mathbf{A r} \mathbf{r}^{\mathbf{F}}$ determined from the experimental $\mathrm{X}$-ray structure and those computed at the B3LYP/cc-pVDZ-(PP) level of theory in the gas-phase. Values due to a second molecule in the asymmetric unit in square brackets. Atom labels are provided in Figure 6 in the main text.

Table S16. The excitation energies (in eV), absorption wavelengths (nm), and oscillator strengths (f) of the lowest ten lying singlet and triplet states for $\mathbf{A r}^{\mathbf{F}}$-Te-6-Ar ${ }^{\mathbf{F}}$ at the TD-B3LYP/cc-pVTZ-(PP) level of theory at the gasphase $\mathrm{S}_{0}$ geometry (as determined at the B3LYP/cc-pVDZ-(PP) level of theory).

Figure S39. HOMO-1, HOMO, LUMO, and LUMO+1, i.e., dominant orbitals involved in the low-lying excited singlet states, for $\mathbf{A} \mathbf{r}^{\mathbf{F}}-\mathbf{T e}-\mathbf{6}-\mathbf{A r} \mathbf{r}^{\mathbf{F}}$ at the TDB3LYP/cc-pVTZ-(PP) level of theory at the gas-phase $\mathrm{S}_{0}$ geometry (as determined at the B3LYP/cc-pVTZ-(PP) level of theory). All orbitals illustrated with an isovalue $=0.02$.

Table S17. The phosphorescence energies (in eV) and corresponding wavelengths (in brackets in $\mathrm{nm}$ ) for select compounds as determined at the (U)B3LYP/ccpVTZ-(PP) level of theory. Phosphorescence energies determined from (i) the singlet to triplet energy gap at the optimized geometry for the triplet state $\left(\mathrm{E}_{\text {vertical }}\right)$; (ii) the energy difference between the lowest energy triplet and ground state singlet at their corresponding optimized geometries ( $\left.E_{\text {adiabatic }}\right)$; or (iii) the energy difference between the lowest energy triplet and ground state singlet at their corresponding optimized geometries including the zero-point vibrational energy difference $\left(\mathrm{E}_{0-0}\right)$.

Table S18. The excitation energies (in eV), absorption wavelengths (nm), and oscillator strengths (f) of the lowest ten lying singlet and triplet states for 4BTe, 4BSe, and $4 \mathbf{B S}$ at the TD-B3LYP/cc-pVTZ-(PP) level of theory at the gas-phase $\mathrm{S}_{0}$ geometry (as determined at the B3LYP/cc-pVTZ-(PP) level of theory).

Table S19. Vertical excitation energies (in eV), oscillator strengths (f), and dominant S-49 transitions for the two lowest lying singlet states for 4BS, 4BSe, and 4BTe, as computed at the TD-B3LYP/aug-cc-pVTZ-(PP) level of theory in THF (IEF-PCM).

Figure S40. Molecular orbitals, as computed at the TD-B3LYP/aug-cc-pVTZ-(PP) level of theory in THF (IEF-PCM), involved in the main excitations for 4BS. All orbitals illustrated with an isovalue $=0.02$.

Figure S41. Molecular orbitals, as computed at the TD-B3LYP/aug-cc-pVTZ-(PP) level of theory in THF (IEF-PCM), involved in the main excitations for 4BSe. All orbitals illustrated with an isovalue $=0.02$. 
Figure S42. Molecular orbitals, as computed at the TD-B3LP/aug-cc-pVTZ-(PP) level of S-50 theory in THF (IEF-PCM), involved in the main excitations for 4BTe. All orbitals illustrated with an isovalue $=0.02$.

Table S20. Gas phase B3LYP/cc-pVTZ (cc-pVTZ-PP Te) determined xyz coordinates S-50 (in $\AA$ ) for TPA-Te-6-TPA at $\mathrm{S}_{0}$ equilibrium geometry.

Table S21. Gas phase B3LYP/cc-pVTZ (cc-pVTZ-PP Te) determined xyz coordinates S-52 (in $\AA$ ) for Benzo-Te(BPin)TPA at $\mathrm{S}_{0}$ equilibrium geometry.

Table S22. Gas phase UB3LYP/cc-pVTZ (cc-pVTZ-PP Te) determined xyz coordinates S-53 (in $\AA$ ) for Benzo-Te(BPin)TPA at $T_{1}$ equilibrium geometry.

Table S23. Gas phase B3LYP/cc-pVTZ (cc-pVTZ-PP Te) determined xyz coordinates S-55 (in $\AA$ ) for $\mathbf{T e C}_{4}(2 \text {-thienyl })_{4}$ at $\mathrm{S}_{0}$ equilibrium geometry.

Table S24. Gas phase B3LYP/cc-pVTZ (cc-pVTZ-PP Te) determined xyz coordinates S-56 (in $\AA$ ) for Naph-Te-6-Naph at $\mathrm{S}_{0}$ equilibrium geometry.

Table S25. Gas phase B3LYP/cc-pVTZ (cc-pVTZ-PP Te) determined xyz coordinates S-57 (in $\AA$ ) for Naph-Te-6-Naph at $\mathrm{T}_{1}$ equilibrium geometry.

Table S26. Gas phase B3LYP/cc-pVTZ (cc-pVTZ-PP Te) determined xyz coordinates S-58 (in $\AA$ ) for Fl-Te-6-Fl at $\mathrm{S}_{0}$ equilibrium geometry.

Table S27. Gas phase B3LYP/cc-pVTZ (cc-pVTZ-PP Te) determined xyz coordinates S-59 (in $\AA$ ) for $\mathbf{A r} \mathbf{r}^{\mathrm{F}}$-Te-6-Ar $\mathbf{r}^{\mathrm{F}}$ at $\mathrm{S}_{0}$ equilibrium geometry.

Table S28. Gas phase UB3LYP/cc-pVTZ (cc-pVTZ-PP Te) determined xyz coordinates S-61 (in $\AA$ ) for $\mathbf{A} \mathbf{r}^{\mathrm{F}}$ - $\mathbf{T e}-\mathbf{6}-\mathbf{A} \mathbf{r}^{\mathrm{F}}$ at $\mathrm{T}_{1}$ equilibrium geometry.

Table S29. B3LYP/cc-pVTZ determined xyz coordinates (in $\AA$ ) for $\mathbf{4 B S}$ in THF at $\mathrm{S}_{0} \quad$ S-62 equilibrium geometry.

Table S30. B3LYP/cc-pVTZ determined xyz coordinates (in $\AA$ ) for 4 BSe in THF at $\mathrm{S}_{0} \quad$ S-63 equilibrium geometry.

Table S31. B3LYP/cc-pVTZ (cc-pVTZ-PP Te) determined xyz coordinates (in $\AA$ ) for S-64 4BTe in THF at $\mathrm{S}_{0}$ equilibrium geometry.

5. References 
1. ${ }^{1} \mathrm{H},{ }^{13} \mathrm{C}\left\{{ }^{1} \mathrm{H}\right\},{ }^{11} \mathrm{~B}\left\{{ }^{1} \mathrm{H}\right\}$ and ${ }^{19} \mathrm{~F}$ NMR Spectra

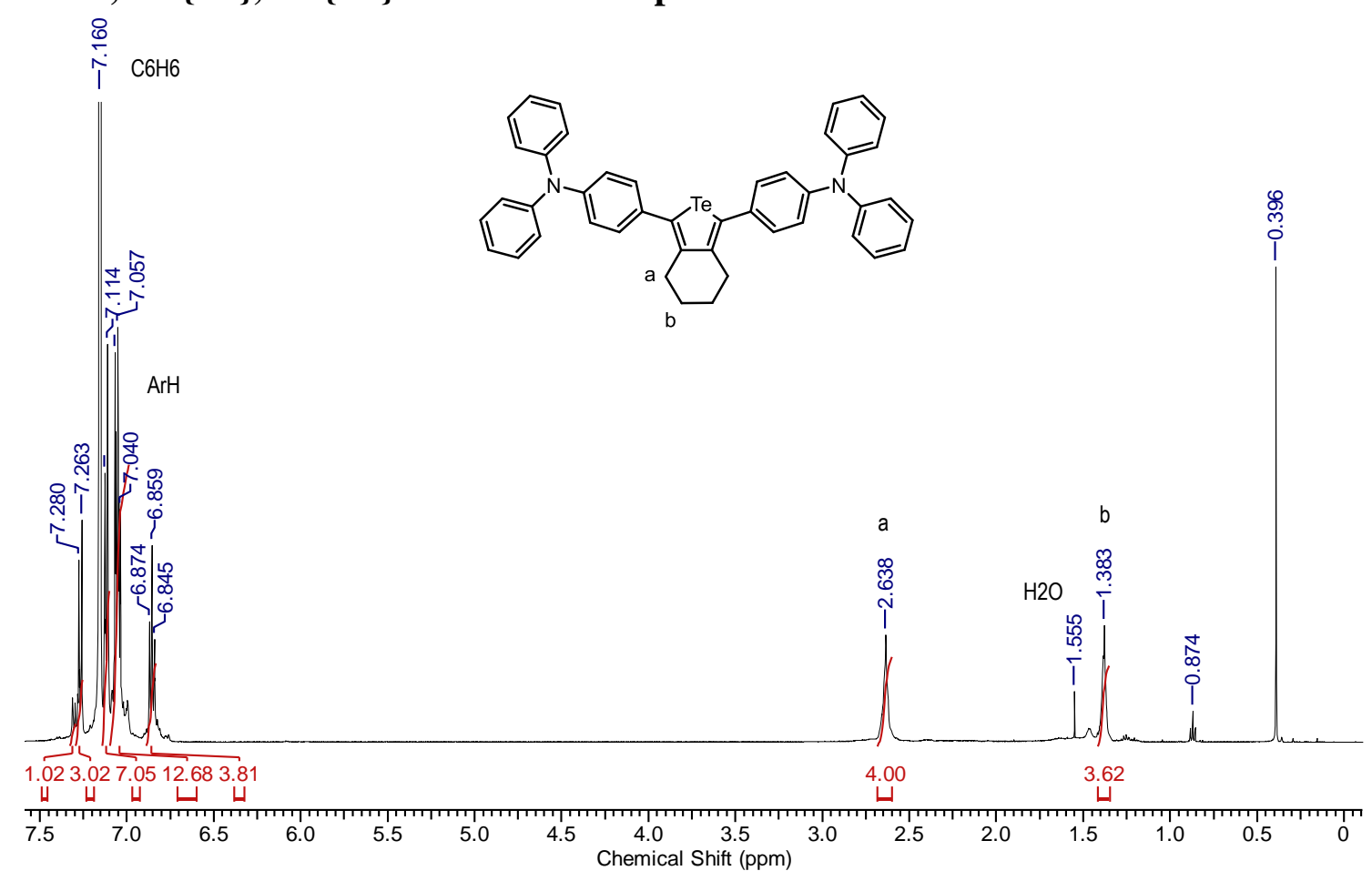

Figure S1. A) ${ }^{1} \mathrm{H}$ NMR spectrum of TPA-Te-6-TPA in $\mathrm{C}_{6} \mathrm{D}_{6}$.

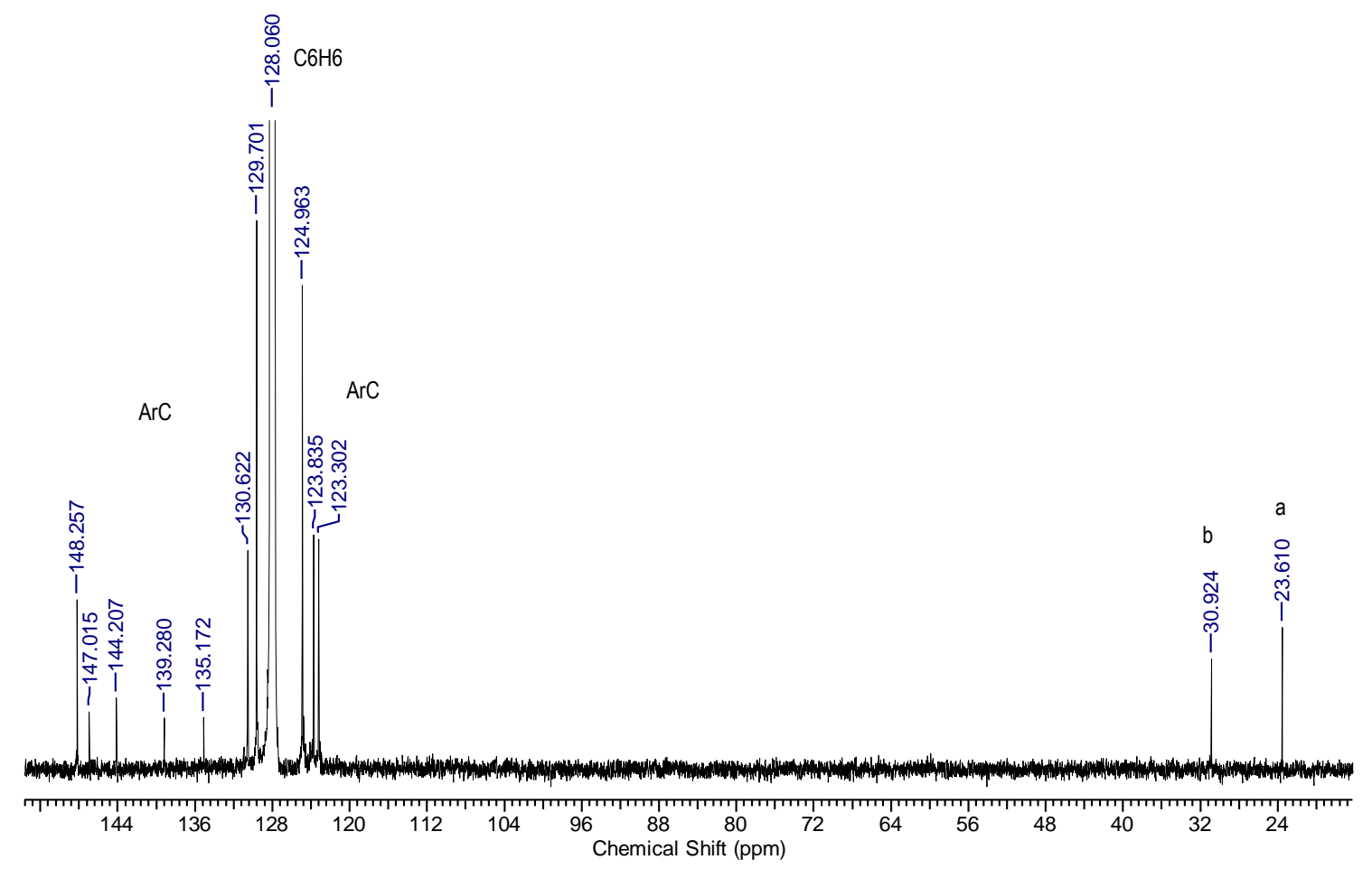

Figure S1. B) ${ }^{13} \mathrm{C}\left\{{ }^{1} \mathrm{H}\right\}$ NMR spectrum of TPA-Te-6-TPA in $\mathrm{C}_{6} \mathrm{D}_{6}$. 

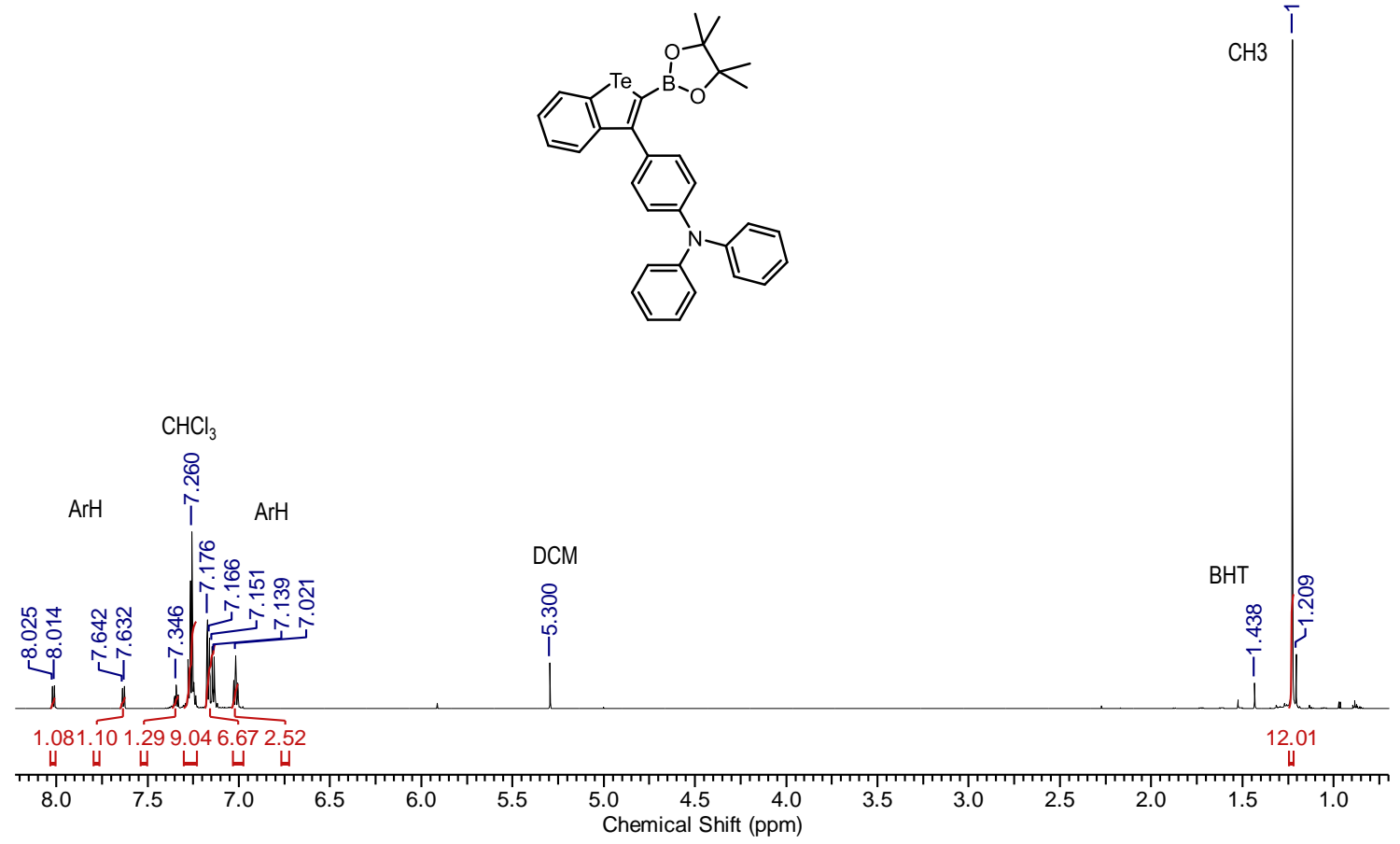

Figure S2. A) ${ }^{1} \mathrm{H}$ NMR spectrum of Benzo-Te(BPin)TPA in $\mathrm{CDCl}_{3}$.

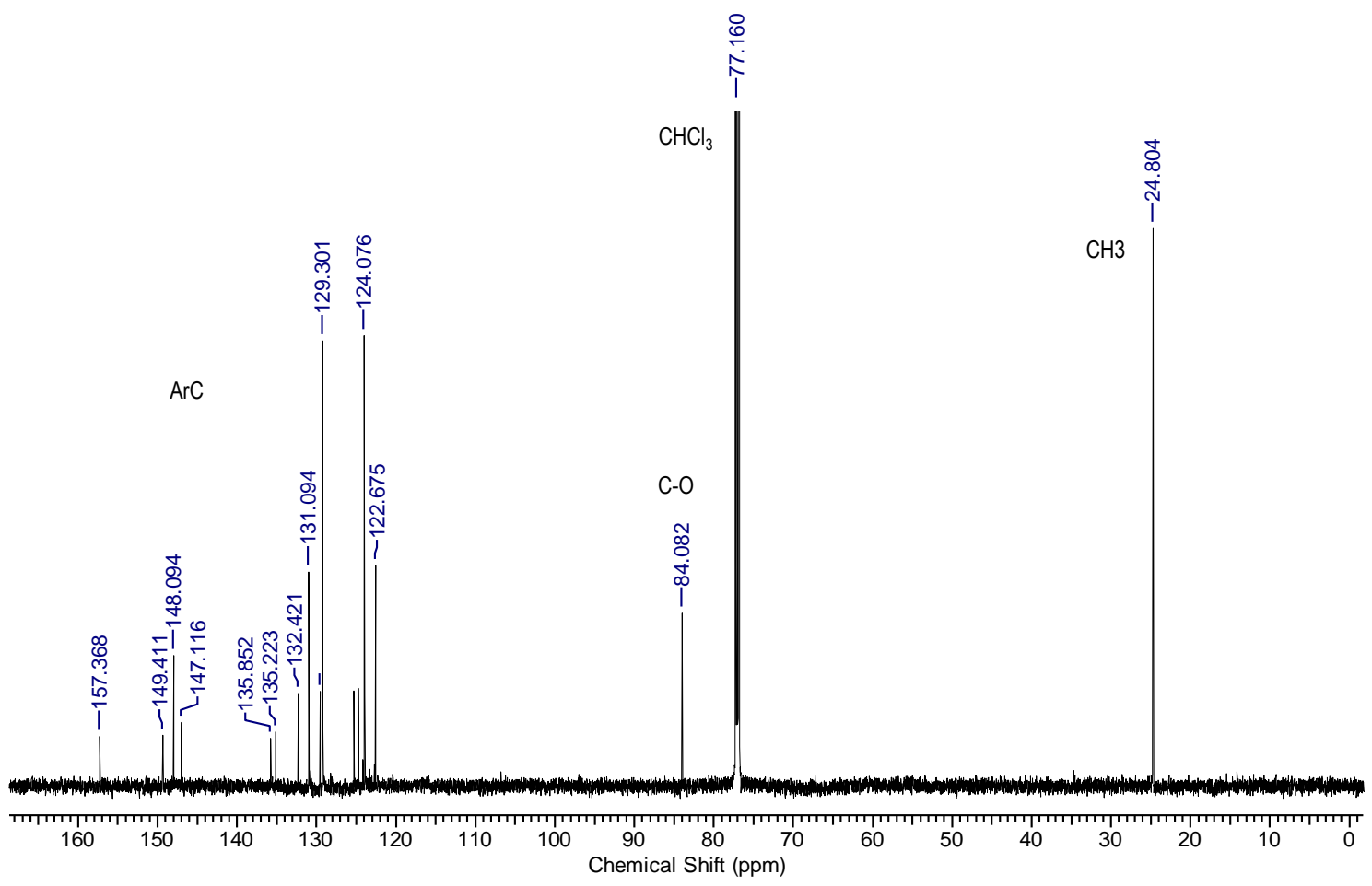

Figure S2. B) ${ }^{13} \mathrm{C}\left\{{ }^{1} \mathrm{H}\right\}$ NMR spectrum of Benzo-Te(BPin)TPA in $\mathrm{CDCl}_{3}$. 


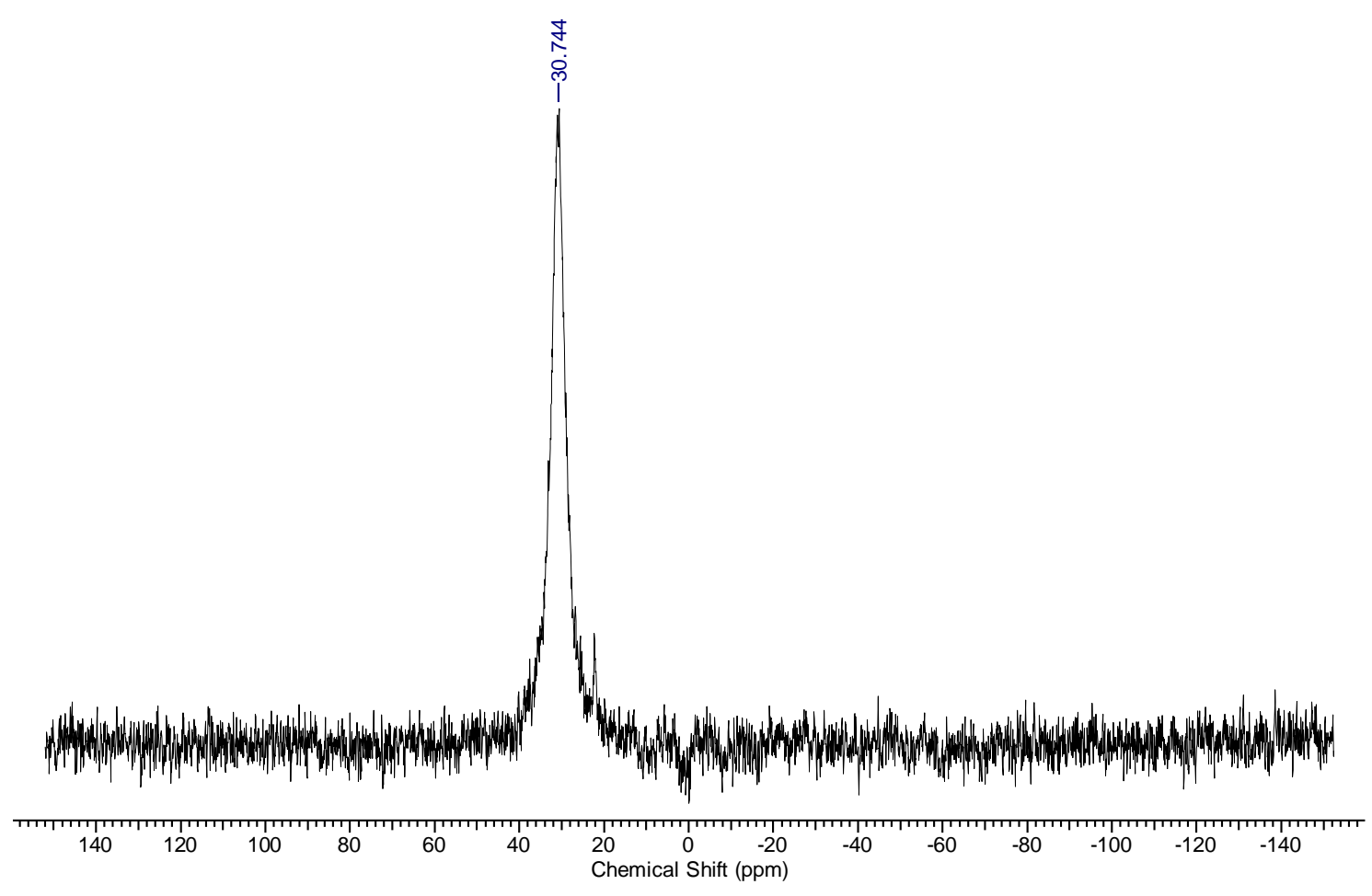

Figure S2. C) ${ }^{11} \mathrm{~B}\left\{{ }^{1} \mathrm{H}\right\}$ NMR spectrum of Benzo-Te(BPin)TPA in $\mathrm{CDCl}_{3}$.

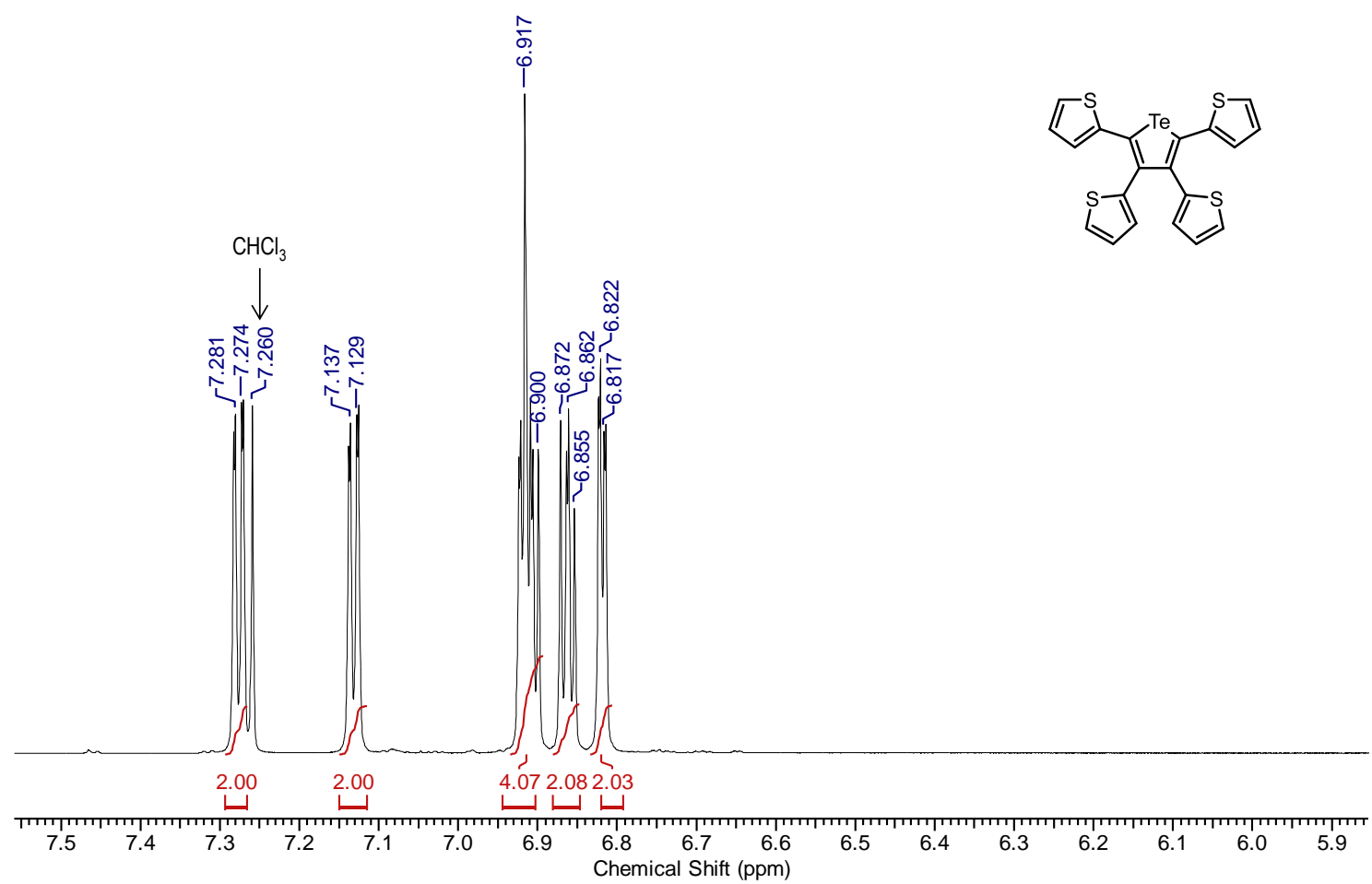

Figure S3. A) ${ }^{1} \mathrm{H}$ NMR spectrum of $\mathbf{T e C}_{4}$ (2-thienyl) ${ }_{4}$ in $\mathrm{CDCl}_{3}$. 


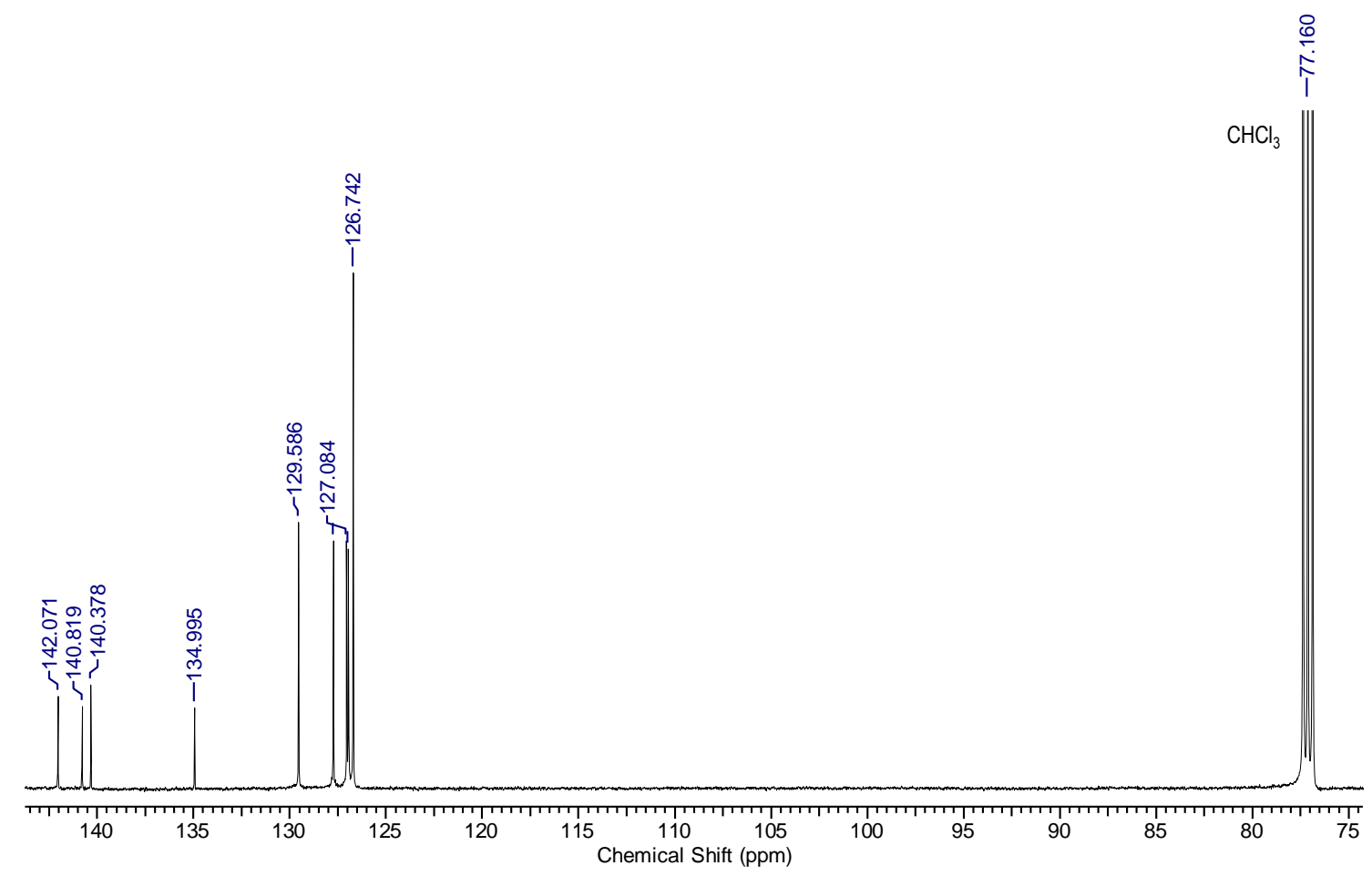

Figure S3. B) ${ }^{13} \mathrm{C}\left\{{ }^{1} \mathrm{H}\right\}$ NMR spectrum of $\mathbf{T e C}_{4}$ (2-thienyl) ${ }_{4}$ in $\mathrm{CDCl}_{3}$.

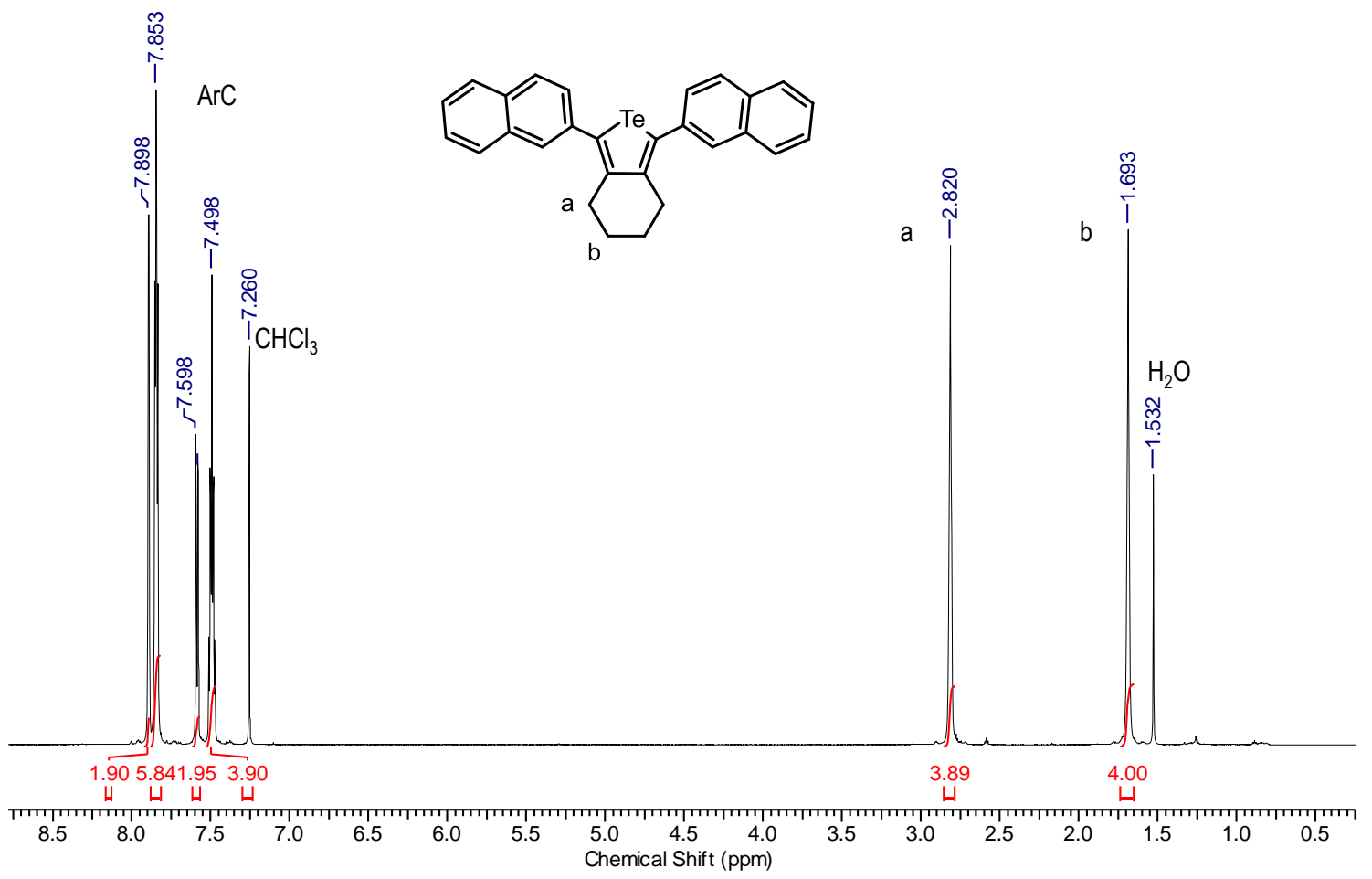

Figure S4. A) ${ }^{1} \mathrm{H}$ NMR spectrum of Naph-Te-6-Naph in $\mathrm{CDCl}_{3}$. 

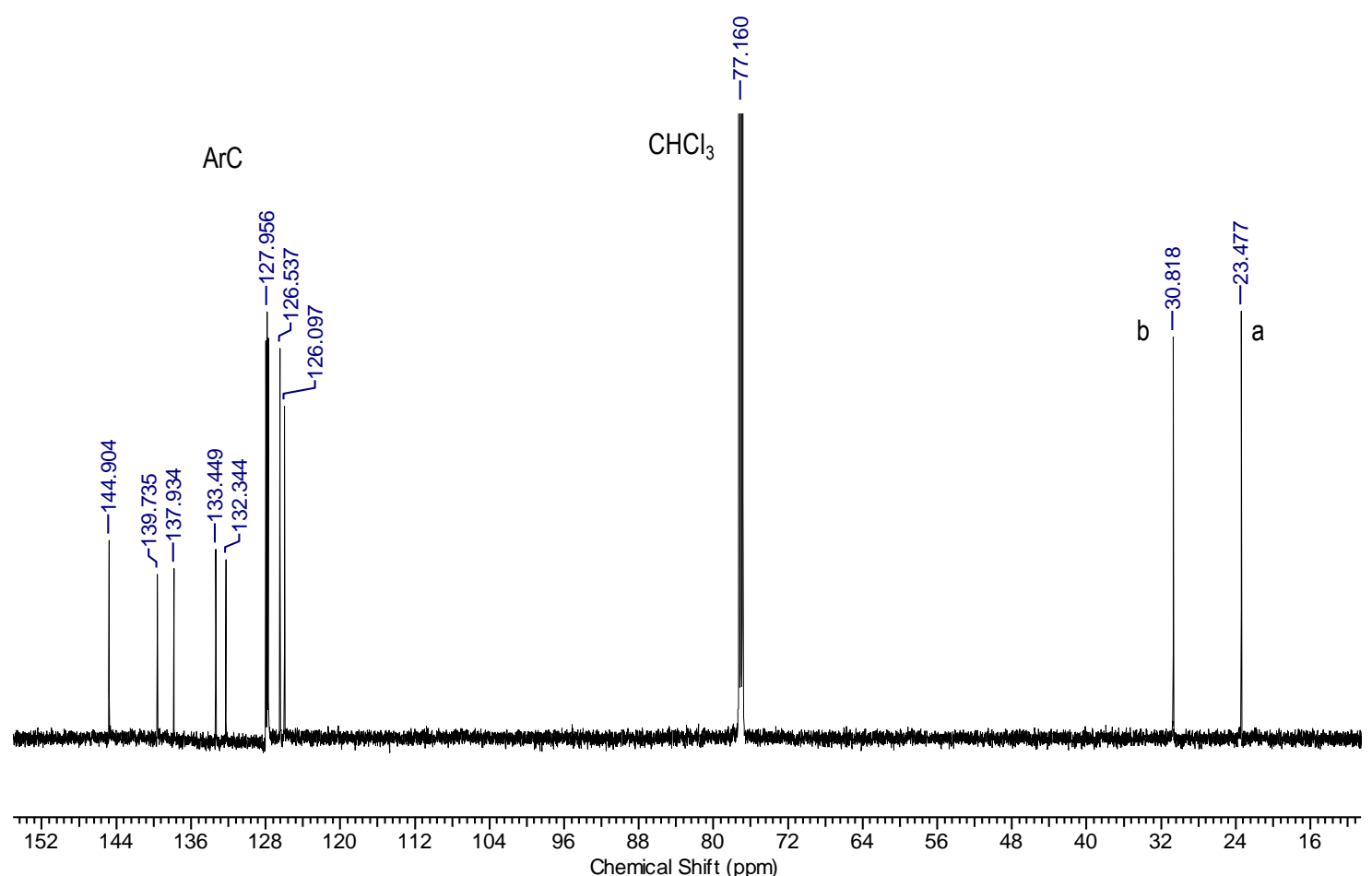

Figure S4. B) ${ }^{13} \mathrm{C}\left\{{ }^{1} \mathrm{H}\right\}$ NMR spectrum of Naph-Te-6-Naph in $\mathrm{CDCl}_{3}$.

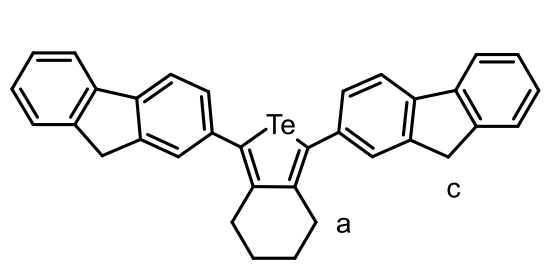

b

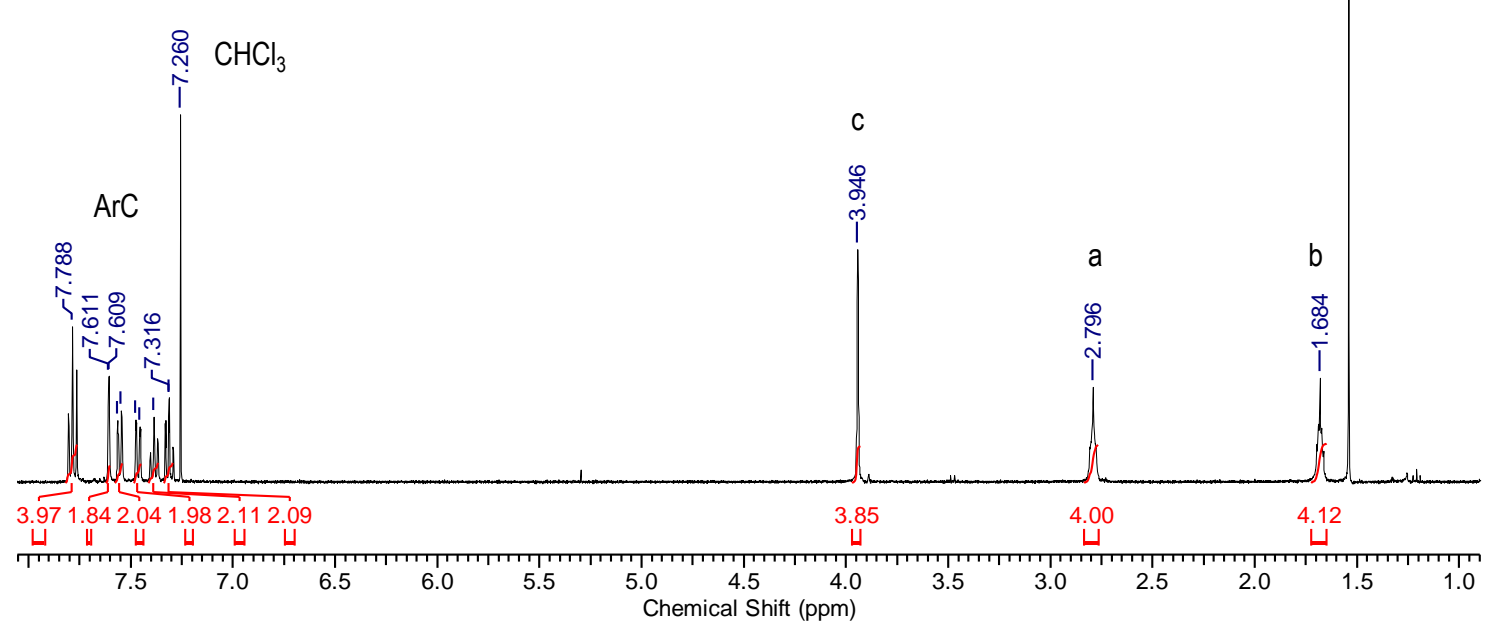

Figure S5. A) ${ }^{1} \mathrm{H}$ NMR spectrum of Fl-Te-6-Fl in $\mathrm{CDCl}_{3}$. 


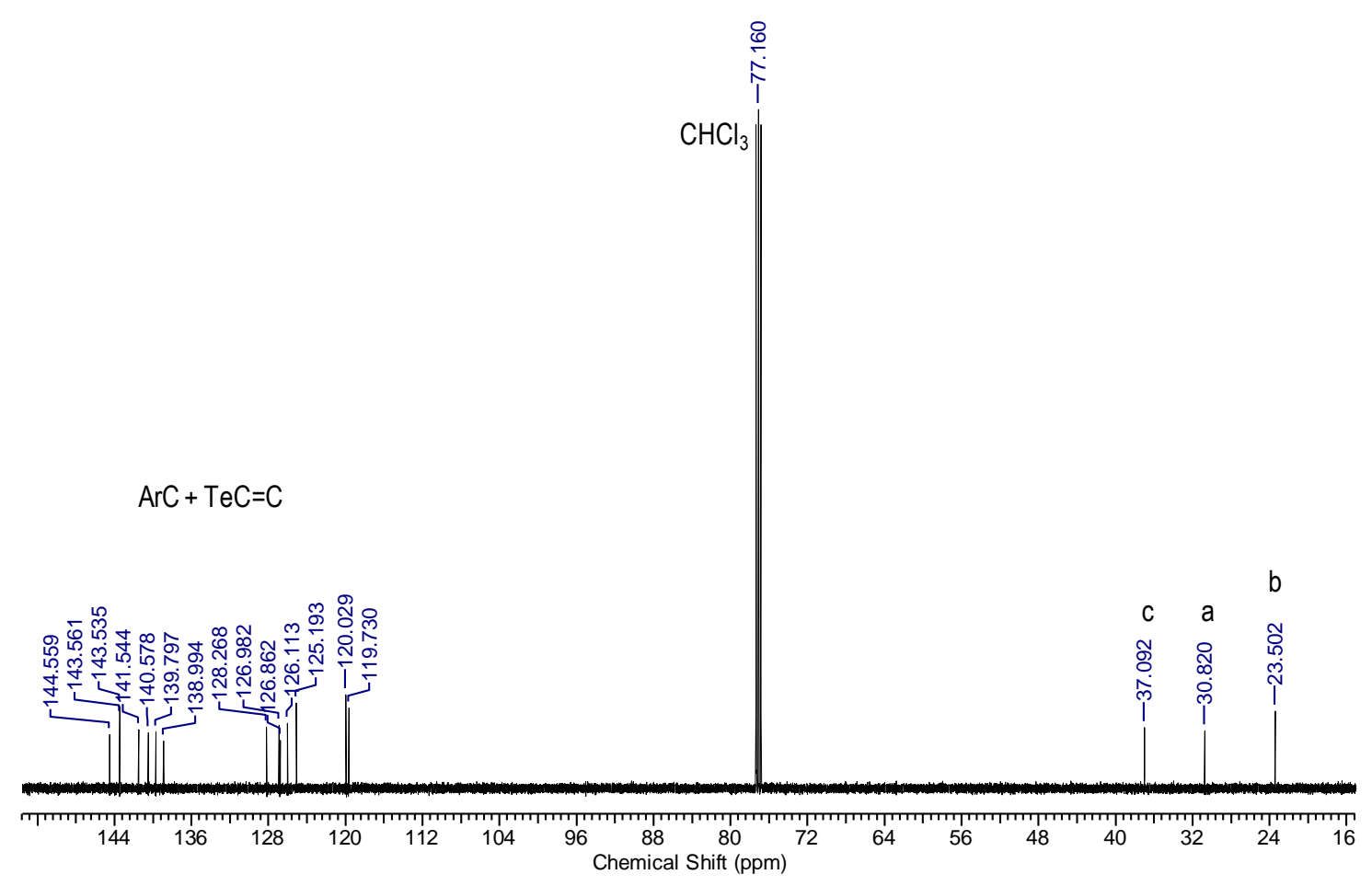

Figure S5. B) ${ }^{13} \mathrm{C}\left\{{ }^{1} \mathrm{H}\right\}$ NMR spectrum of Fl-Te-6-Fl in $\mathrm{CDCl}_{3}$.
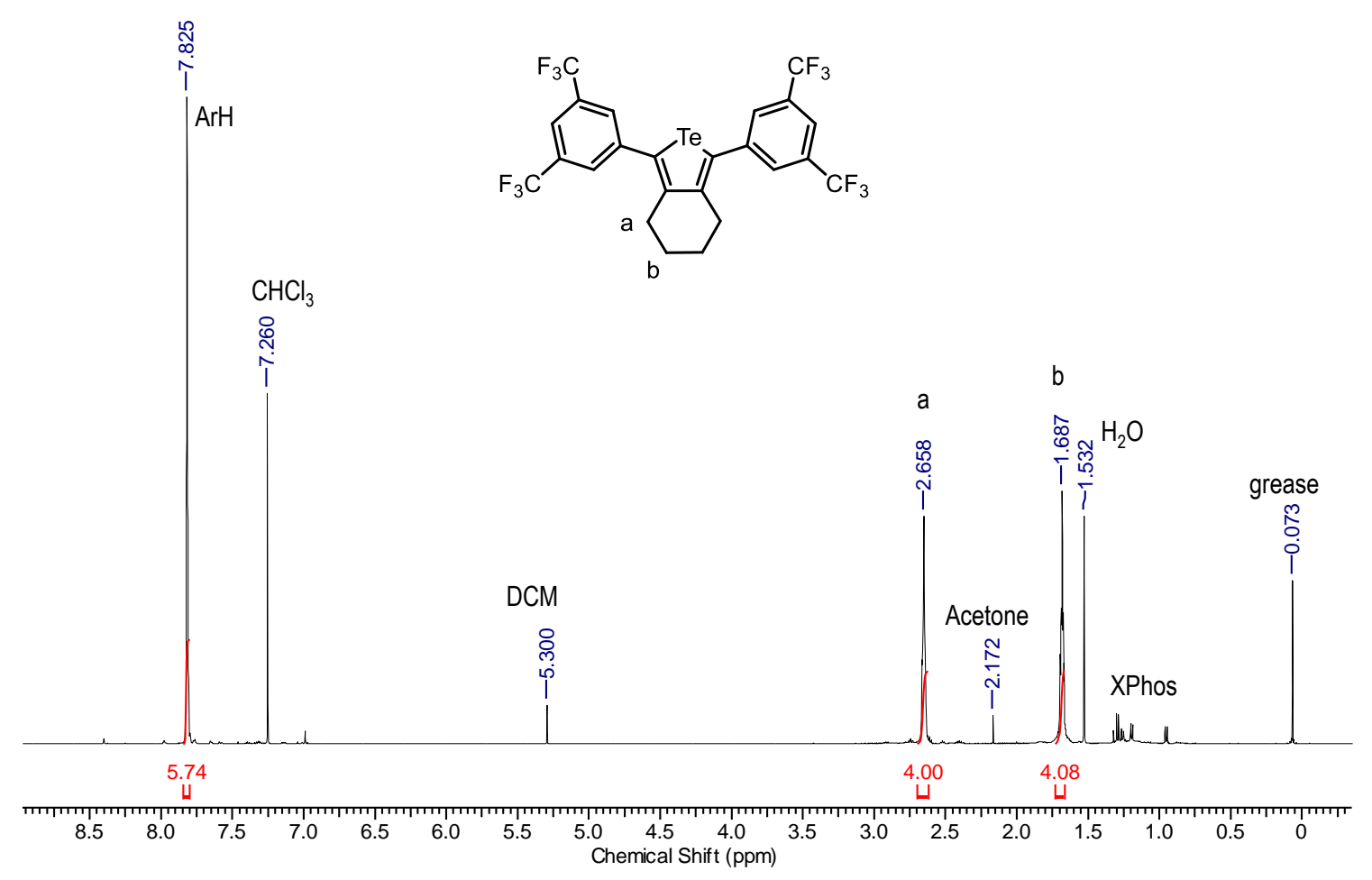

Figure S6. A) ${ }^{1} \mathrm{H}$ NMR spectrum of $\mathbf{A r} \mathbf{r}^{\mathbf{F}}-\mathbf{T e}-\mathbf{6}-\mathbf{A r} \mathbf{r}^{\mathbf{F}}$ in $\mathrm{CDCl}_{3}$. 


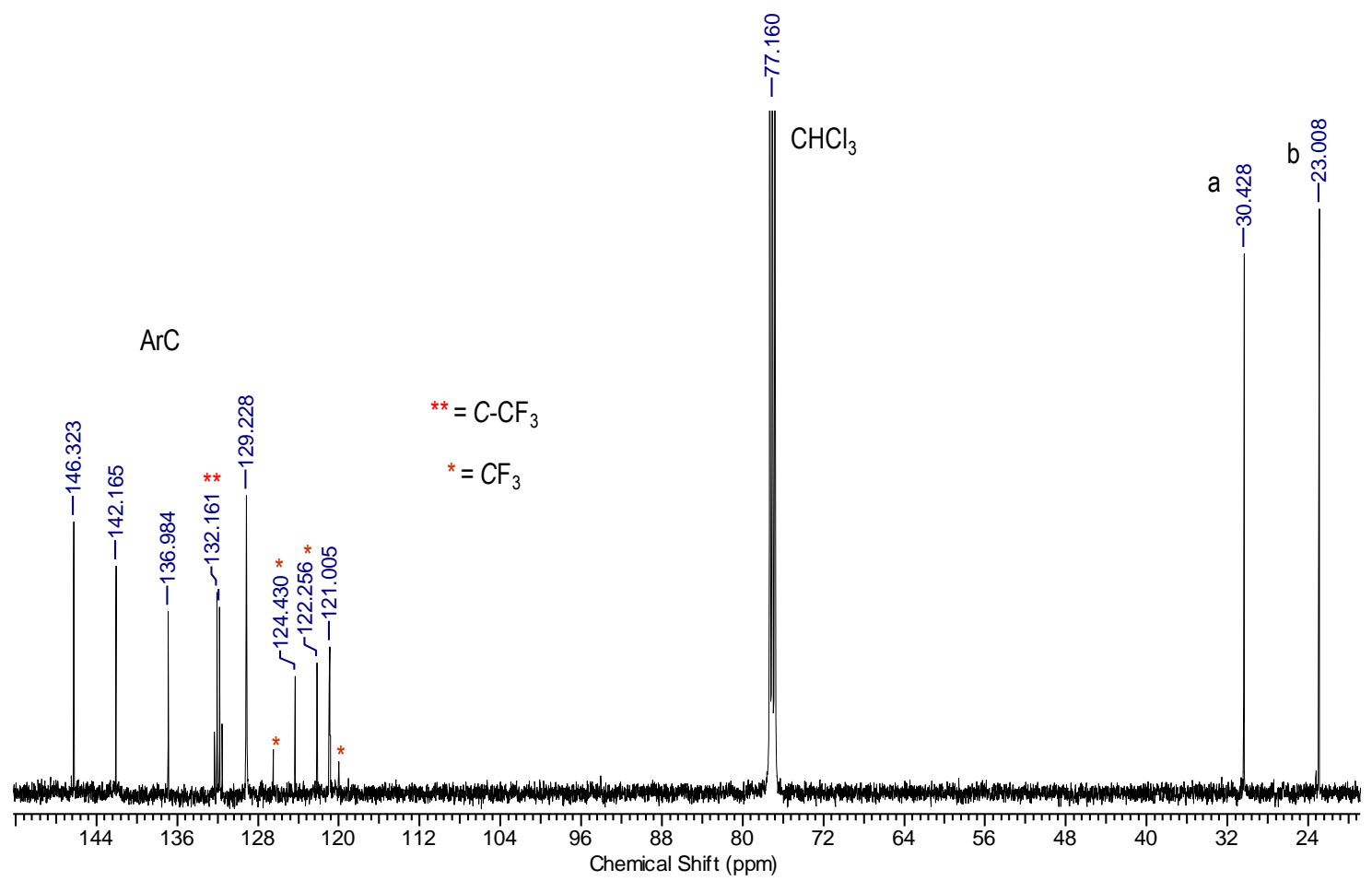

Figure S6. B) ${ }^{13} \mathrm{C}\left\{{ }^{1} \mathrm{H}\right\}$ NMR spectrum of $\mathbf{A} \mathbf{r}^{\mathbf{F}}-\mathbf{T e}-\mathbf{6}-\mathbf{A} \mathbf{r}^{\mathbf{F}}$ in $\mathrm{CDCl}_{3}$.

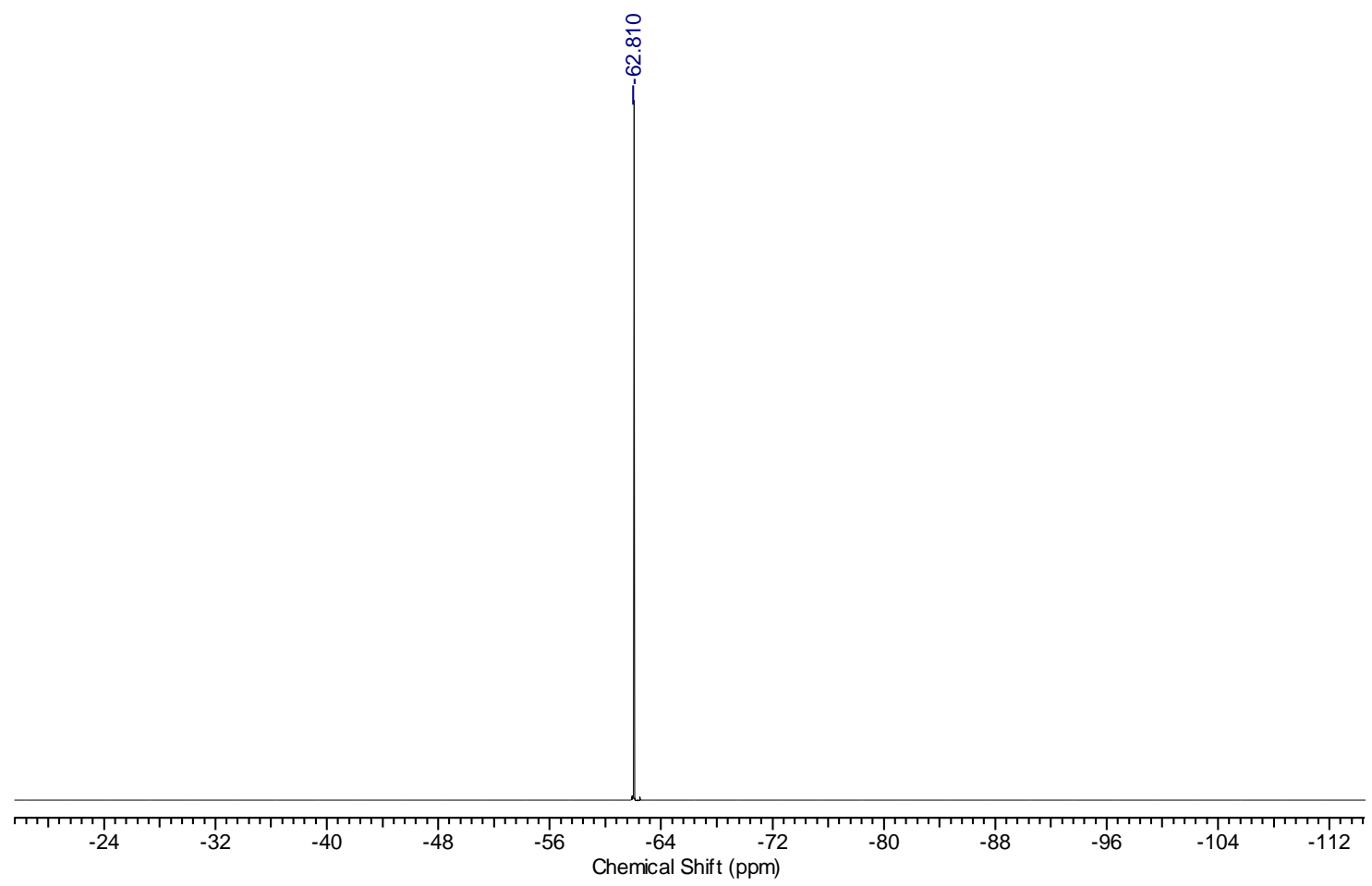

Figure S6. C) ${ }^{19} \mathrm{~F}$ NMR spectrum of $\mathbf{A r} \mathbf{r}^{\mathbf{F}}$-Te-6- $\mathbf{A} \mathbf{r}^{\mathbf{F}}$ in $\mathrm{CDCl}_{3}$. 
2. Absorption, Luminescence and Thermal Data

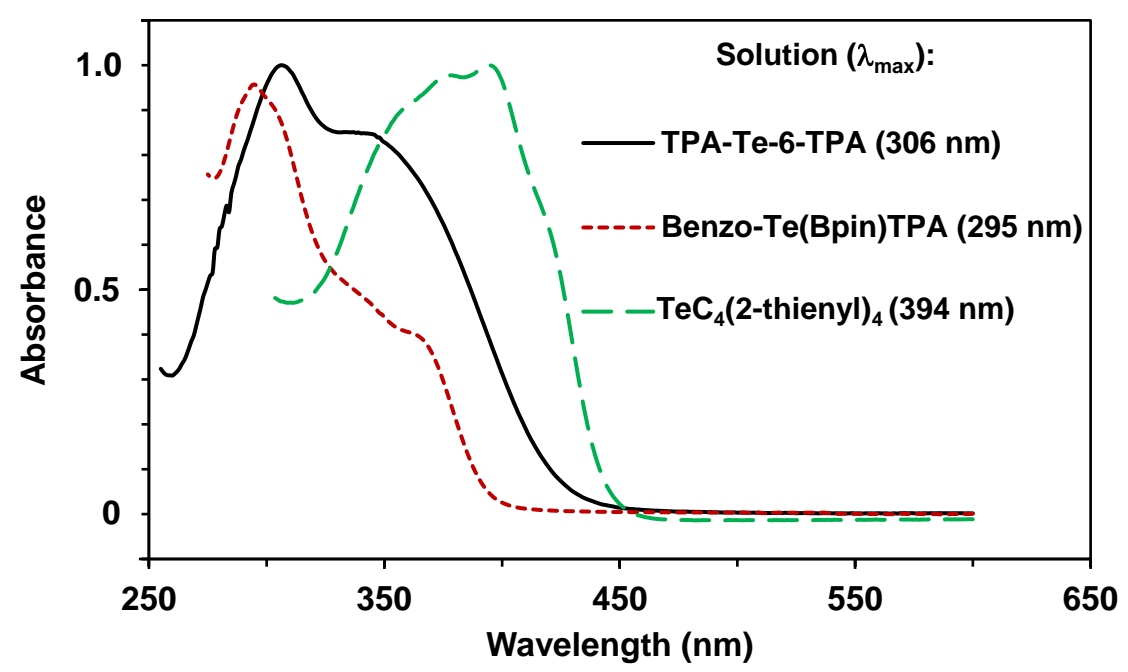

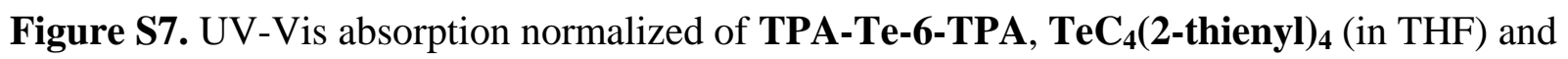
Benzo-Te(BPin)TPA (in cyclohexane).

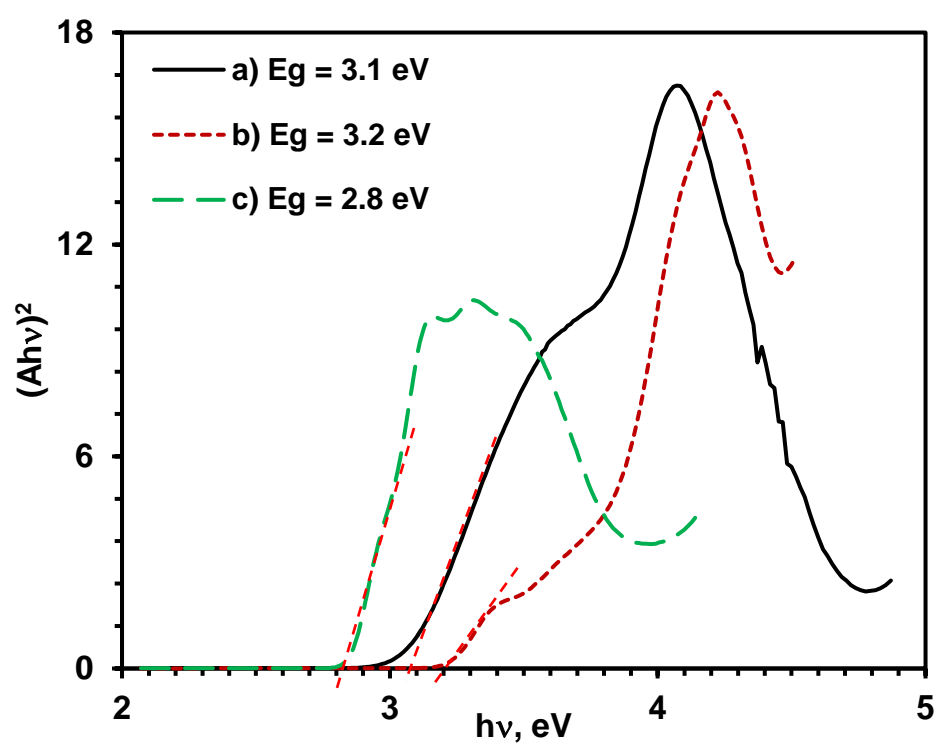

Figure S8. Tauc plots of a) TPA-Te-6-TPA, b) Benzo-Te(BPin)TPA* and c) $\mathbf{T e}_{4}(2 \text {-thienyl })_{4}$ in THF and cyclohexane*. Inset: calculated optical band gaps. 


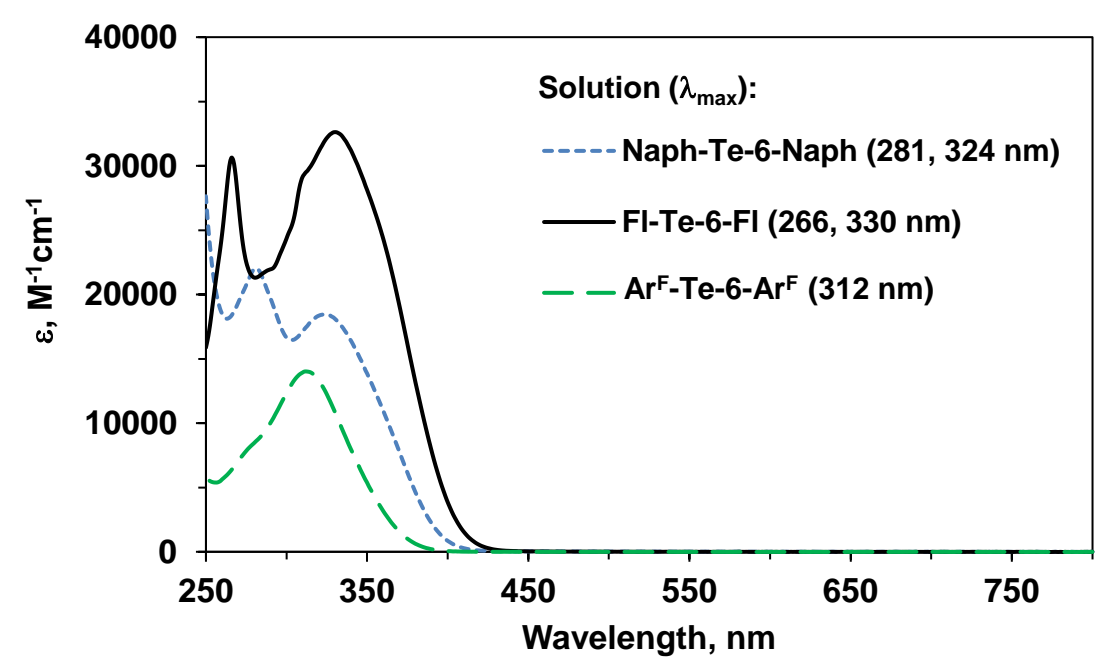

Figure S9. UV-Vis absorption of Naph-Te-6-Naph, Fl-Te-6-Fl and $\mathbf{A r}^{\mathrm{F}}-\mathbf{T e}-\mathbf{6}-\mathbf{A r}^{\mathrm{F}}$ in THF (30 $\mu \mathrm{M})$ at room temperature under $\mathrm{N}_{2}$.

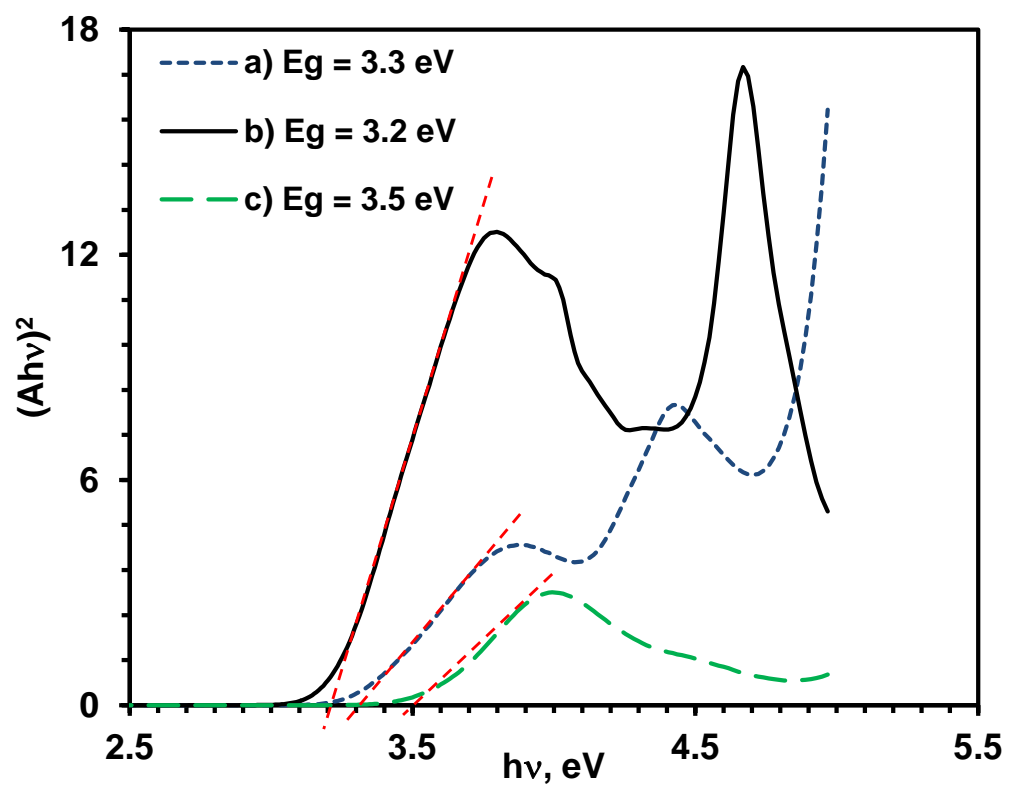

Figure S10. Tauc plots of a) Naph-Te-6-Naph b) Fl-Te-6-Fl and c) $\mathbf{A r}^{\mathrm{F}}-\mathrm{Te}-6-\mathbf{A r}^{\mathrm{F}}$ in THF. Inset: calculated optical band gaps. 


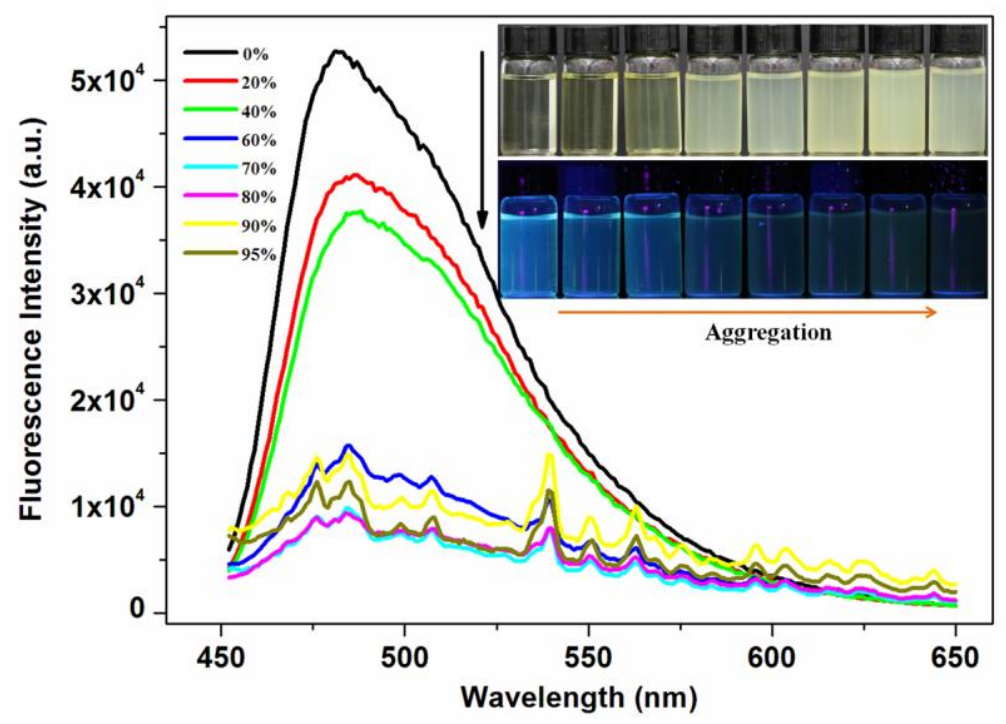

Figure S11. Emission intensity of TPA-Te-6-TPA in different THF/water ratios (listed as \% water content). Inset: aggregates under UV light $\left(\lambda_{\mathrm{ex}}=365 \mathrm{~nm}\right)$. [TPA-Te-6-TPA] $=10^{-4} \mathrm{M}$.

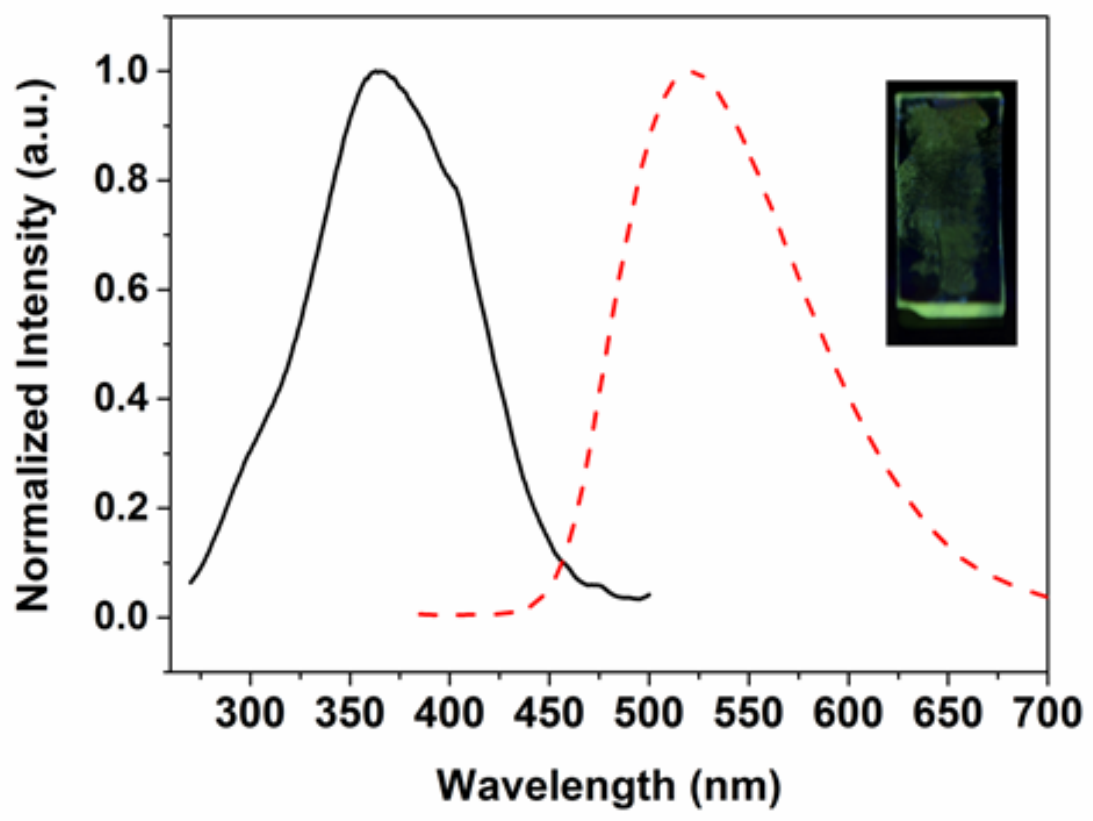

Figure S12. Excitation (solid lines) and emission spectra (dashed lines) of TPA-Te-6-TPA in the solid state. $\lambda_{\mathrm{ex}}=365 \mathrm{~nm}, \lambda_{\mathrm{em}}=525 \mathrm{~nm}$. 


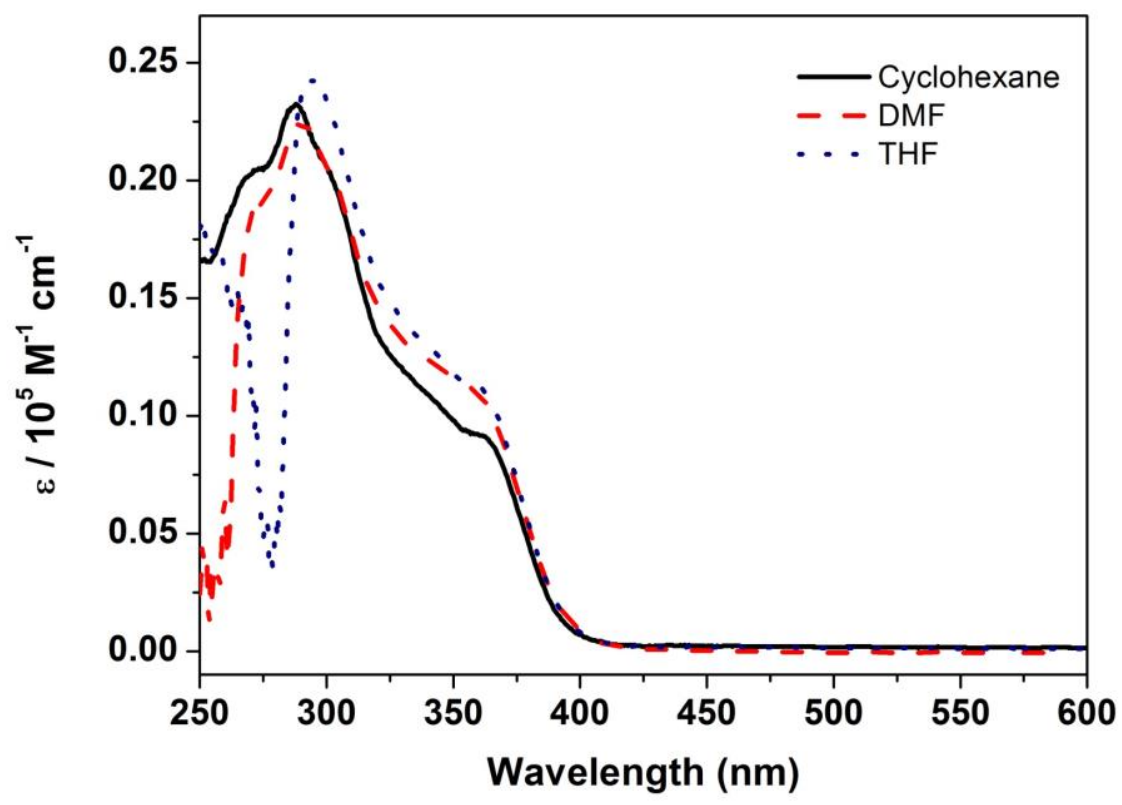

Figure S13. UV-Vis absorption of Benzo-Te(BPin)TPA in THF, cyclohexane and DMF.

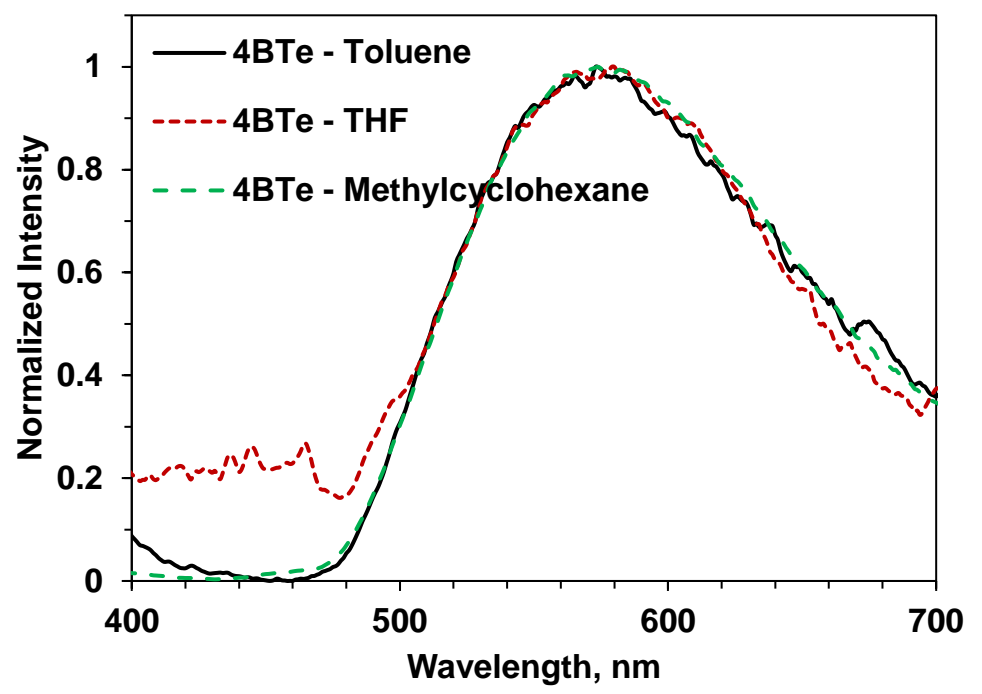

Figure S14. Luminescence measurements $\left(\lambda_{\mathrm{ex}}=350 \mathrm{~nm}\right)$ of $4 \mathrm{BTe}$ in toluene, THF and methylcyclohexane $(10 \mu \mathrm{M})$ after degassing. 


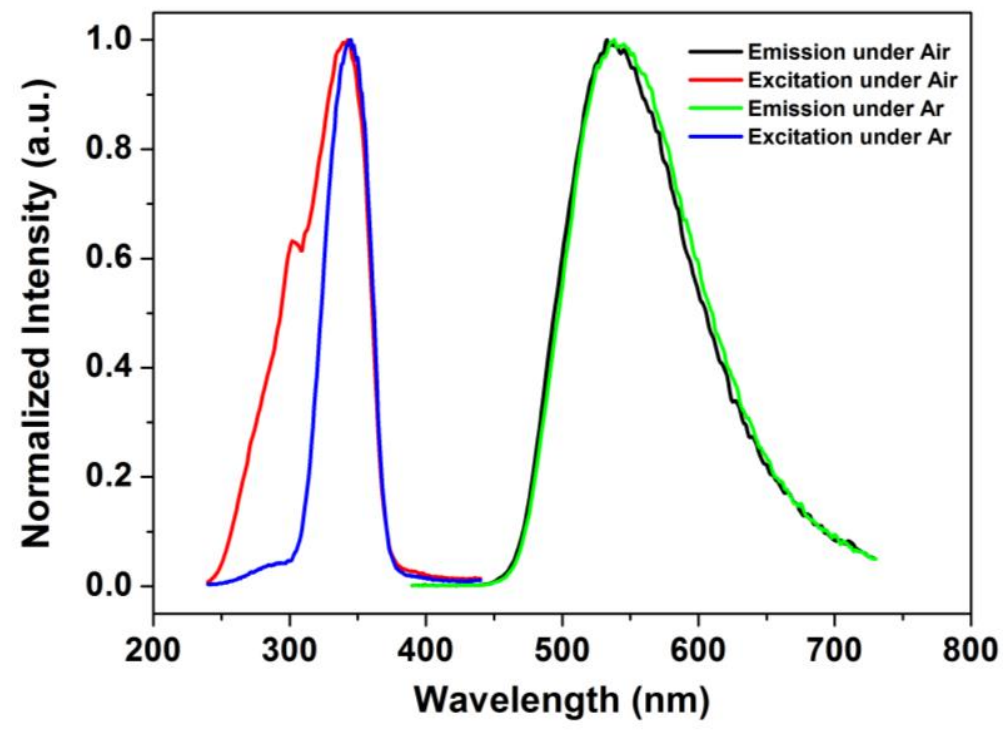

Figure S15. Luminescence measurements of a 4BTe film under air and Ar at $\lambda_{\mathrm{ex}}=370 \mathrm{~nm}$.

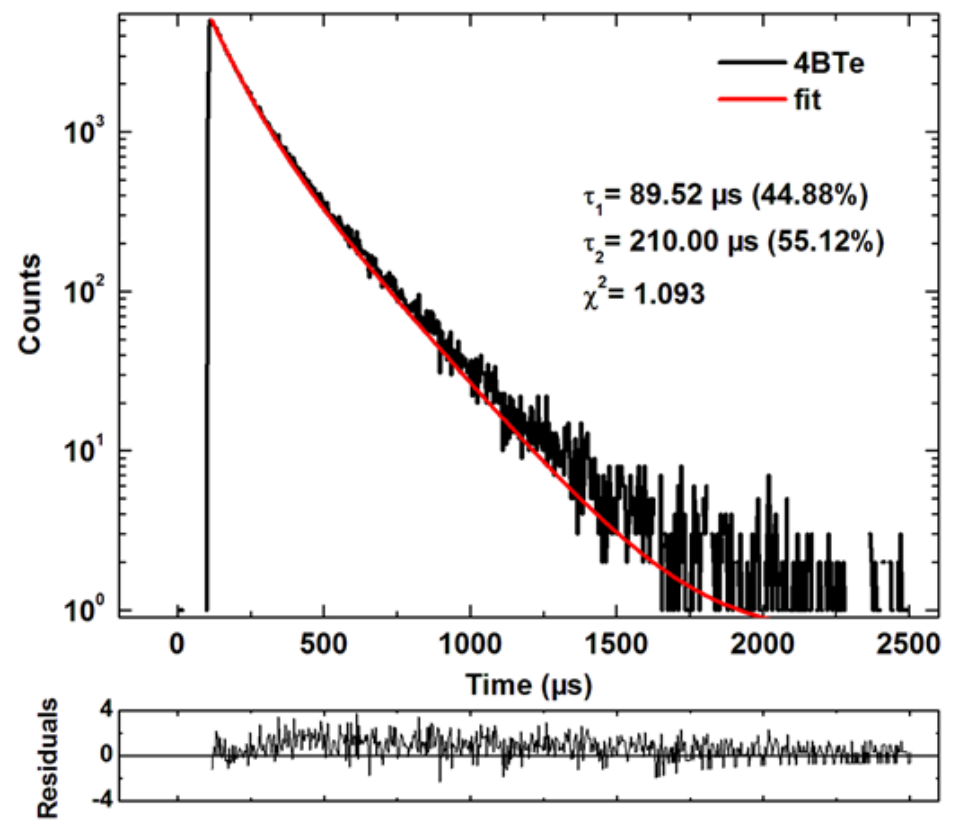

Figure S16. Solid state phosphorescence lifetimes of 4BTe under air, modeled with biexponential decays. 


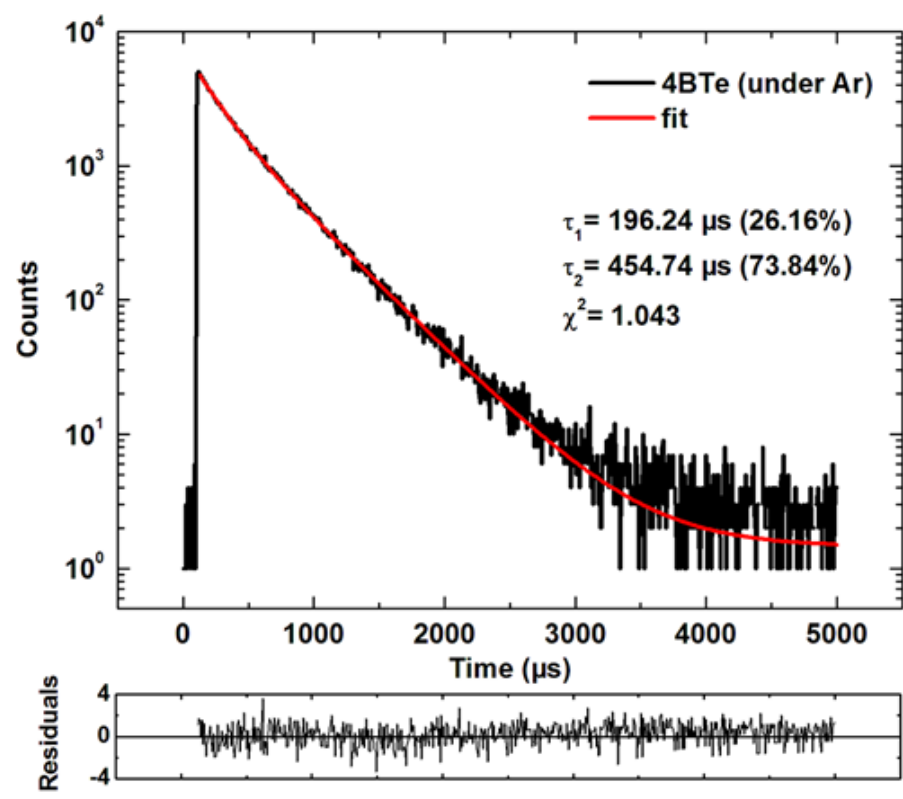

Figure S17. Solid state phosphorescence lifetimes of 4BTe under Ar, modeled with biexponential decays.

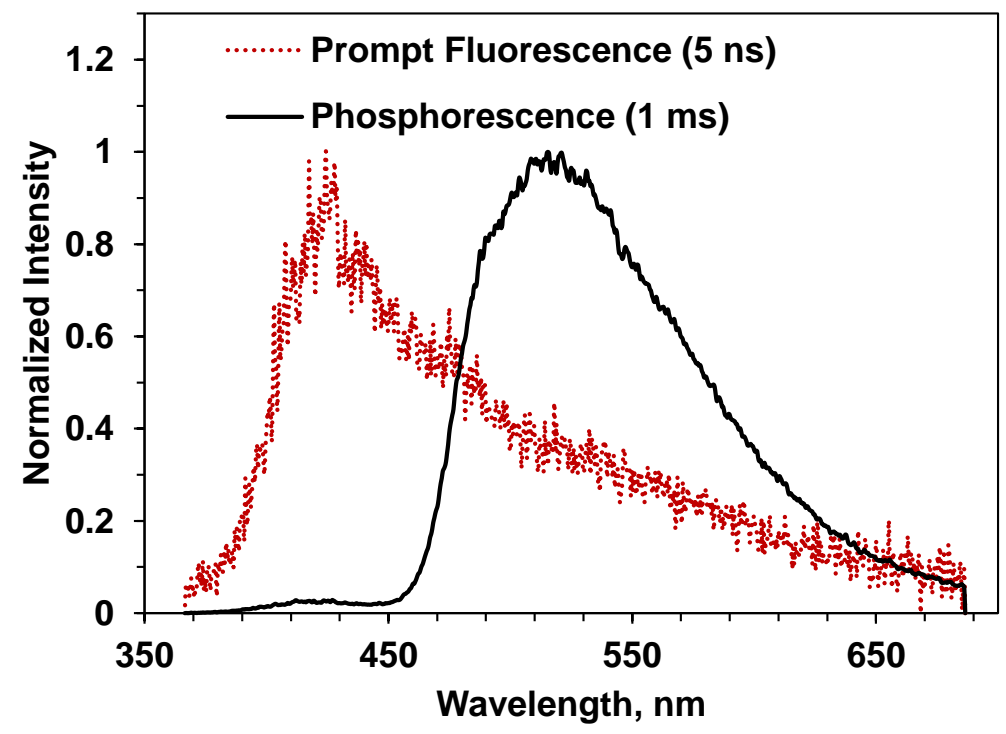

Figure S18. Solid state phosphorescence of 4BTe $(1 \mathrm{wt} \%)$ in a Zeonex ${ }^{\circledR}$ matrix at $300 \mathrm{~K} . \lambda_{\text {em }}=$ $426 \mathrm{~nm} . \tau=1.06 \mathrm{~ns} . \lambda_{\mathrm{em}}=520 \mathrm{~nm} . \tau=242.96 \mu \mathrm{s}$. 


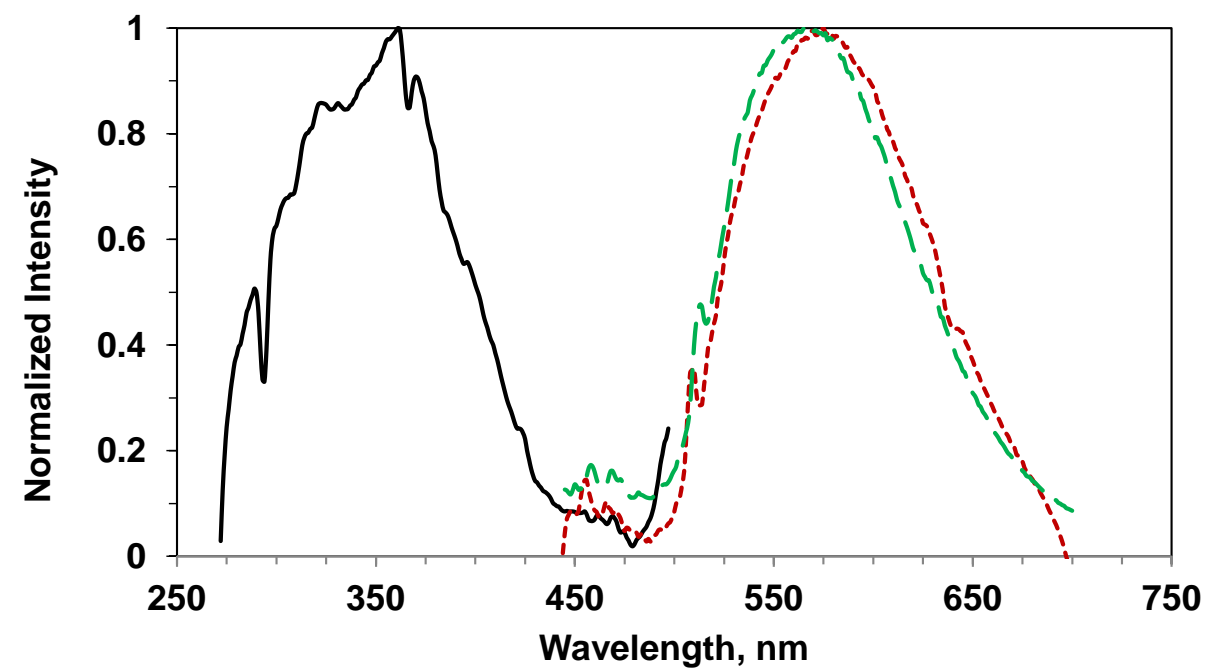

_Excitation -----Emission - - Emission with no PMMA

Figure S19. Solid state phosphorescence of $\mathbf{A r} \mathbf{r}^{\mathbf{F}}-\mathbf{T e}-\mathbf{6}-\mathbf{A r} \mathbf{r}^{\mathbf{F}}$ (32 wt\%) both in a PMMA matrix and in pure film at rt. $\lambda_{\mathrm{ex}}=360 \mathrm{~nm}$. $\lambda_{\mathrm{em}}=567$ and $574 \mathrm{~nm}$, respectively.

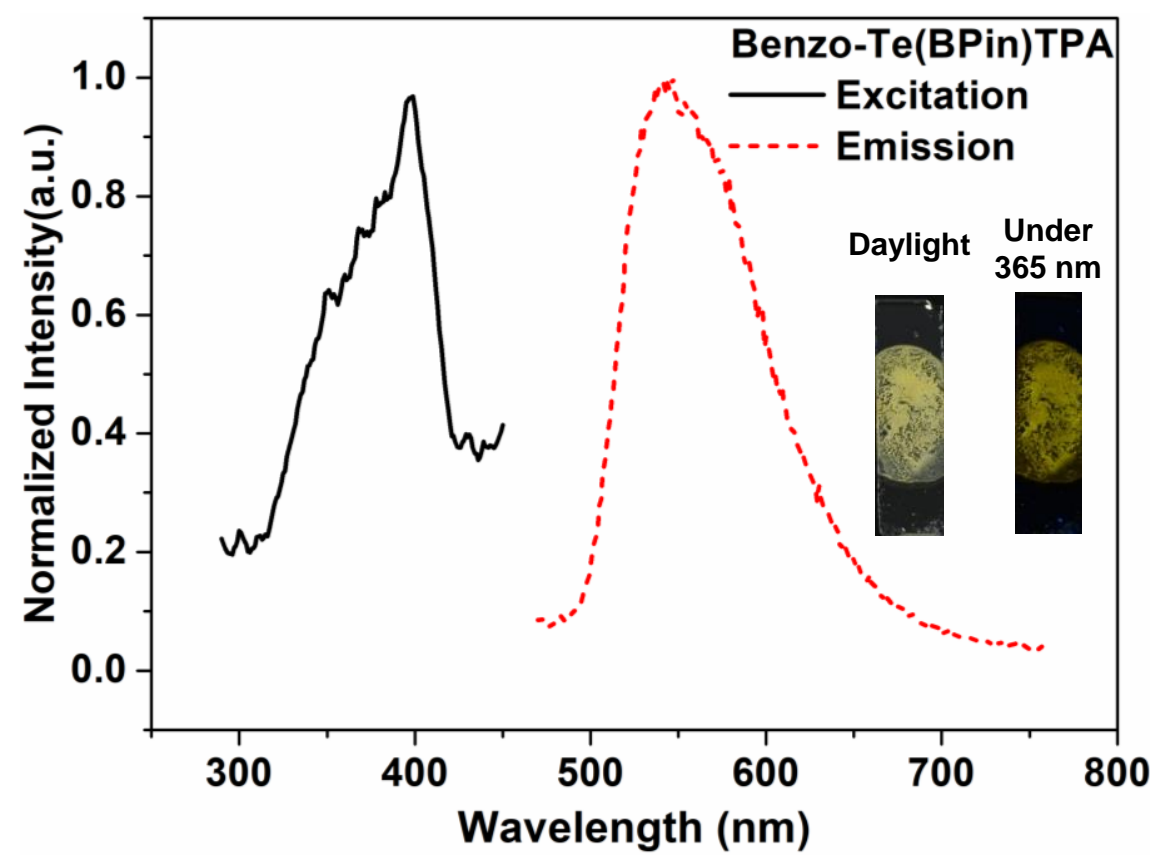

Figure S20. Solid state phosphorescence of Benzo-Te(BPin)TPA measured at rt, $\lambda_{\mathrm{ex}}=360 \mathrm{~nm}$. 


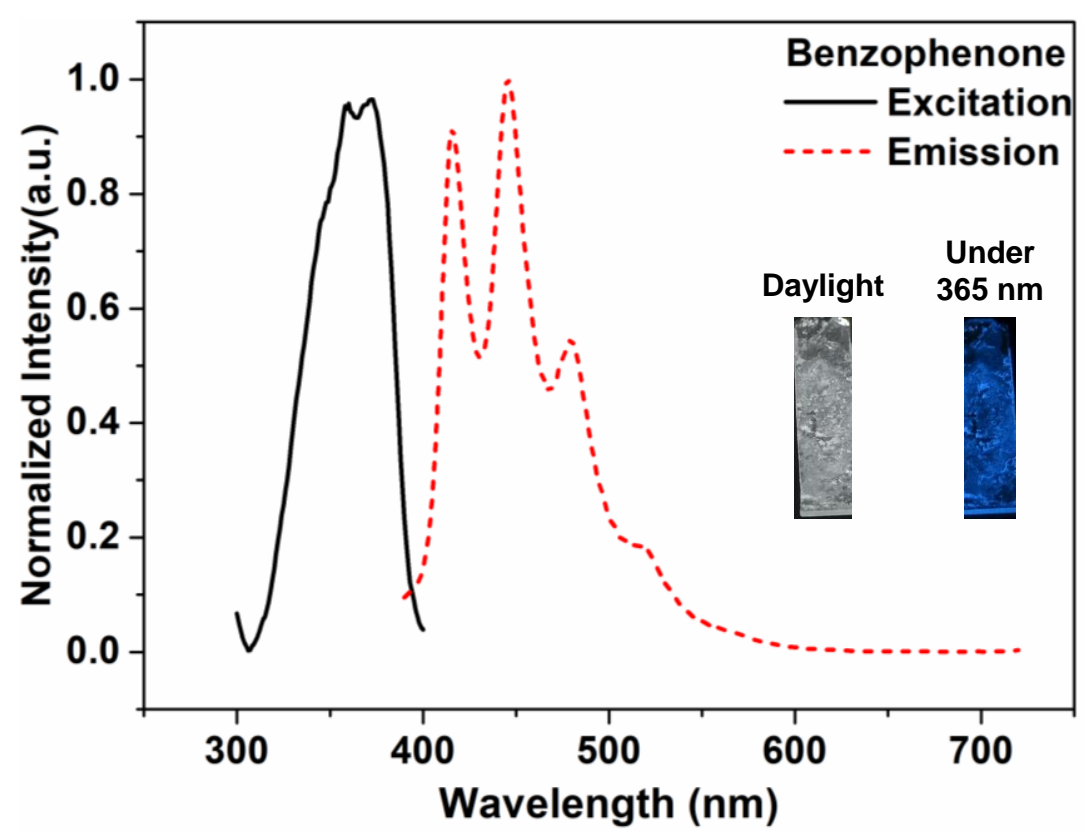

Figure S21. Solid state phosphorescence of benzophenone measured at rt, $\lambda_{\mathrm{ex}}=360 \mathrm{~nm}$.

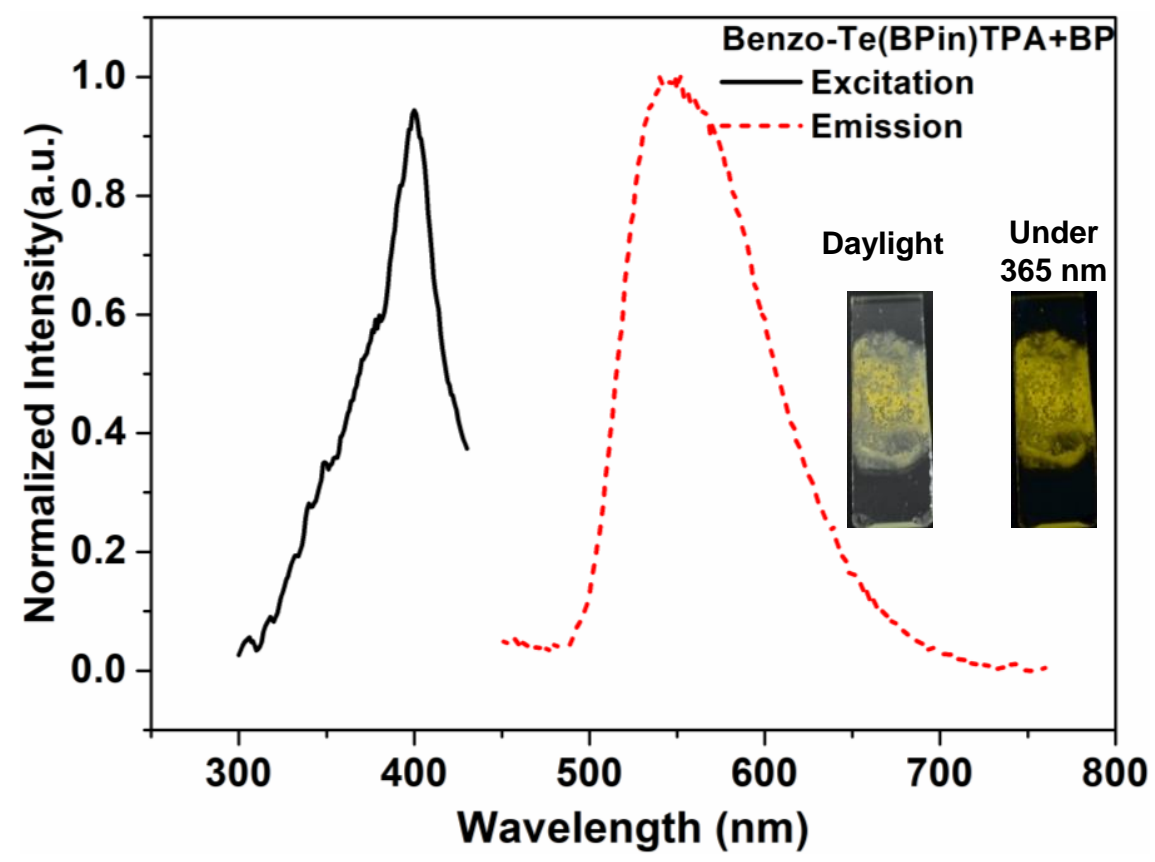

Figure S22. Solid state luminescence of benzophenone (BP) and Benzo-Te(BPin)TPA mixture (1:1) measured at rt, $\lambda_{\mathrm{ex}}=360 \mathrm{~nm}$. 


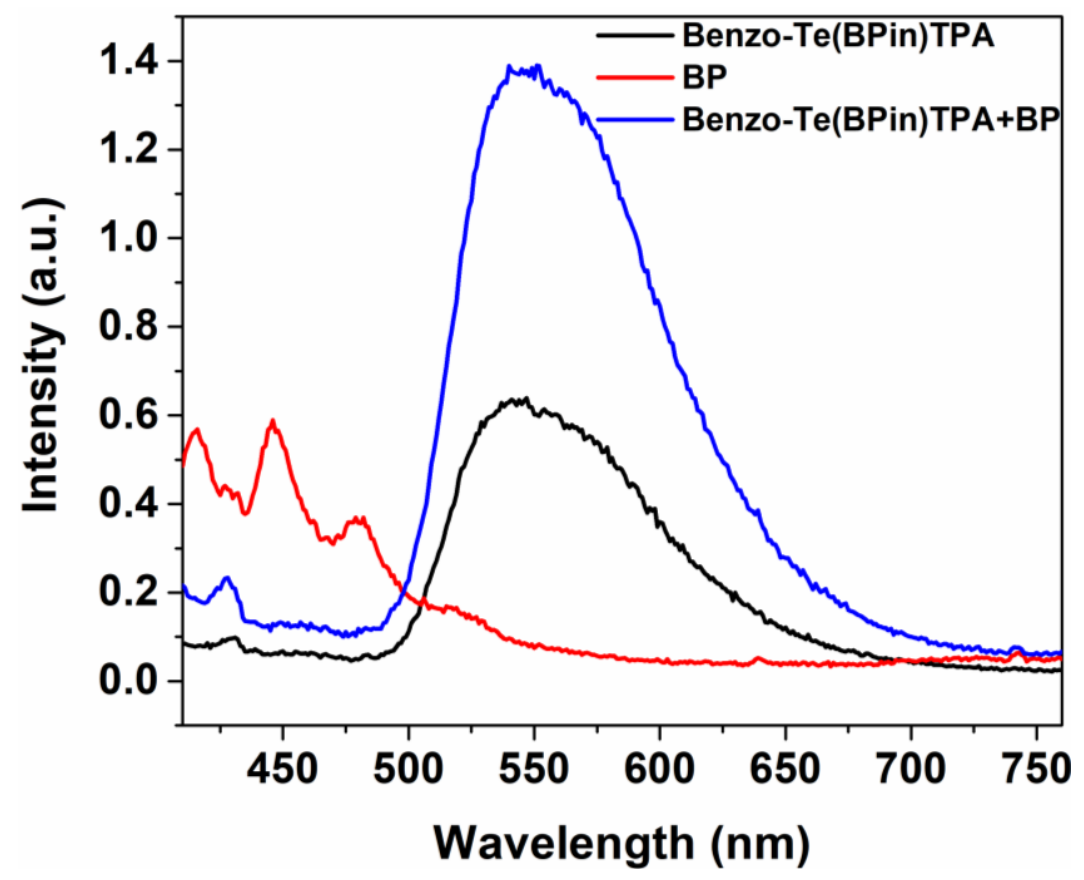

Figure S23. Solid state emission of benzophenone (BP), Benzo-Te(BPin)TPA and their mixture (1:1) measured at $\mathrm{rt}, \lambda_{\mathrm{ex}}=360 \mathrm{~nm}$.

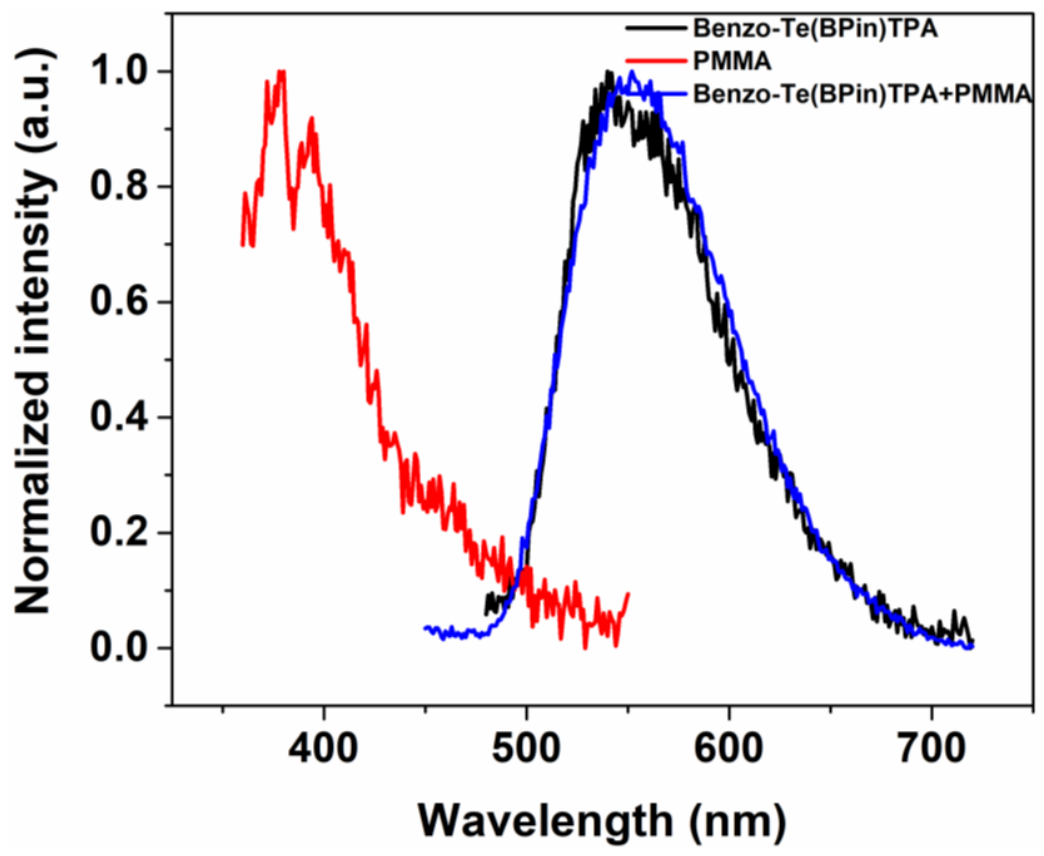

Figure S24. Solid state emission of Benzo-Te(BPin)TPA, PMMA and their mixture measured at $\mathrm{rt}, \lambda_{\mathrm{ex}}=360 \mathrm{~nm}$. 


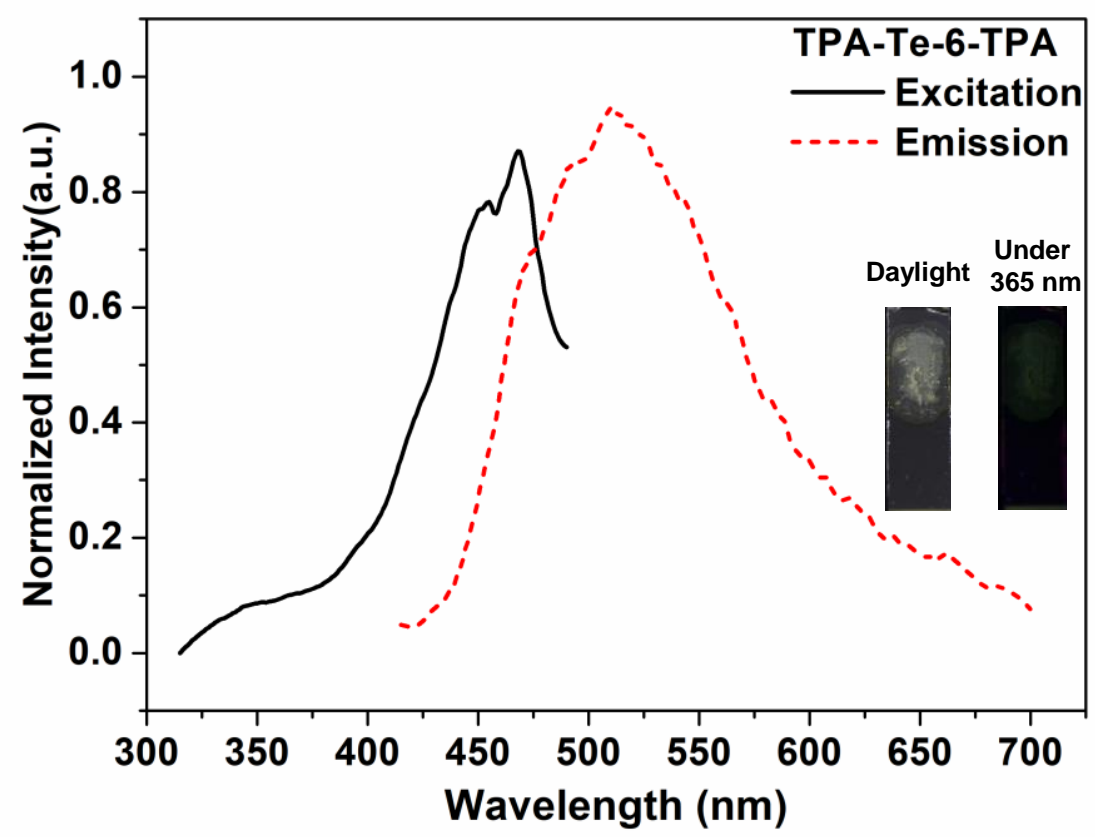

Figure S25. Solid state luminescence of TPA-Te-6-TPA measured at rt, $\lambda_{\mathrm{ex}}=365 \mathrm{~nm}$.

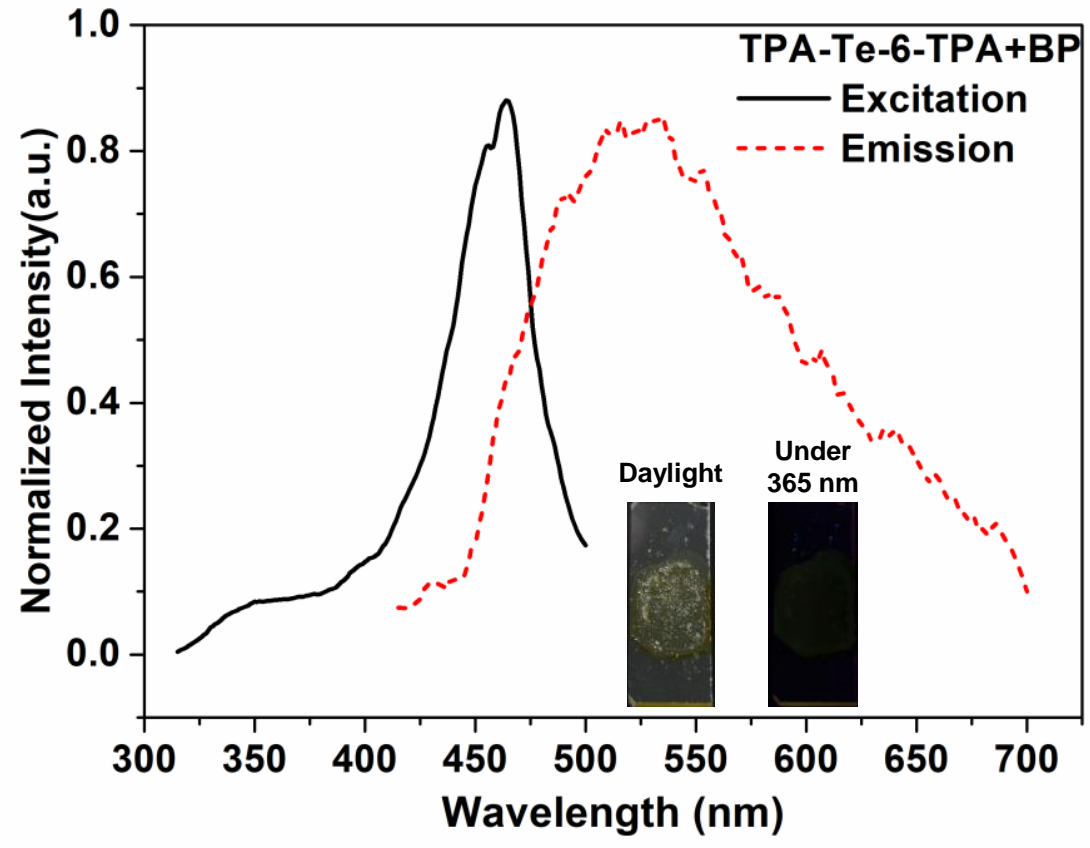

Figure S26. Solid state luminescence of a 1:1 mixture of TPA-Te-6-TPA and benzophenone (BP) measured at rt, $\lambda_{\mathrm{ex}}=365 \mathrm{~nm}$. 


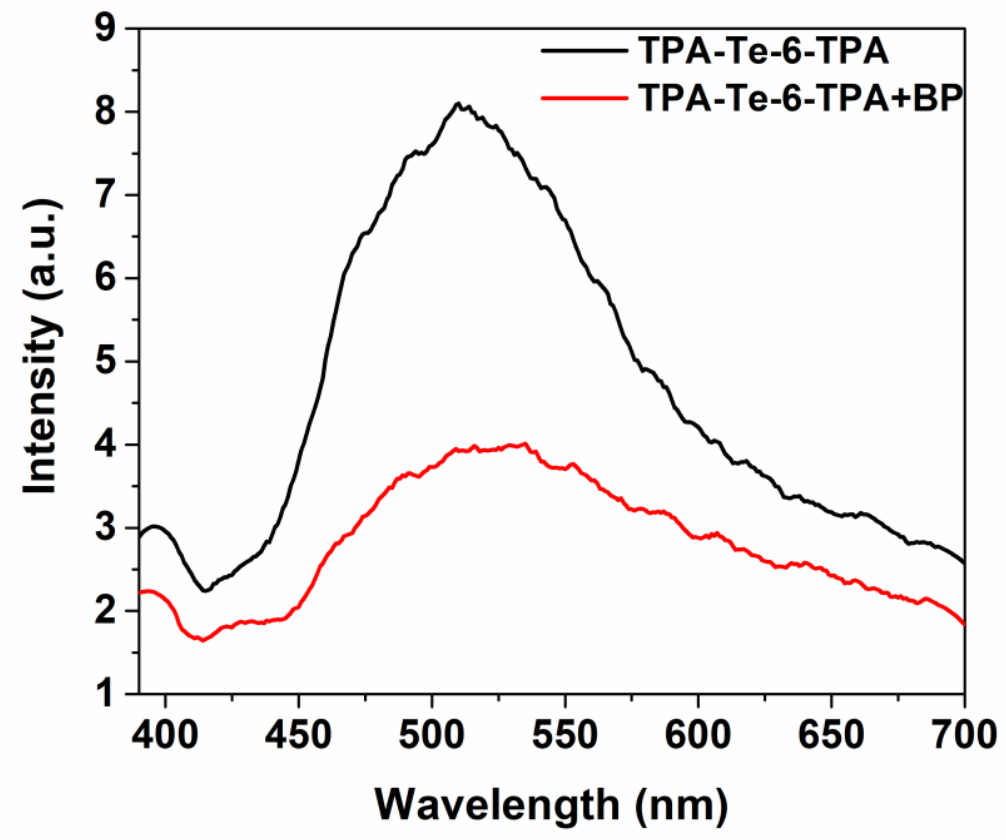

Figure S27. Solid-state emission of TPA-Te-6-TPA and its 1:1 mixture with benzophenone (BP) measured at $\mathrm{rt}, \lambda_{\mathrm{ex}}=365 \mathrm{~nm}$.

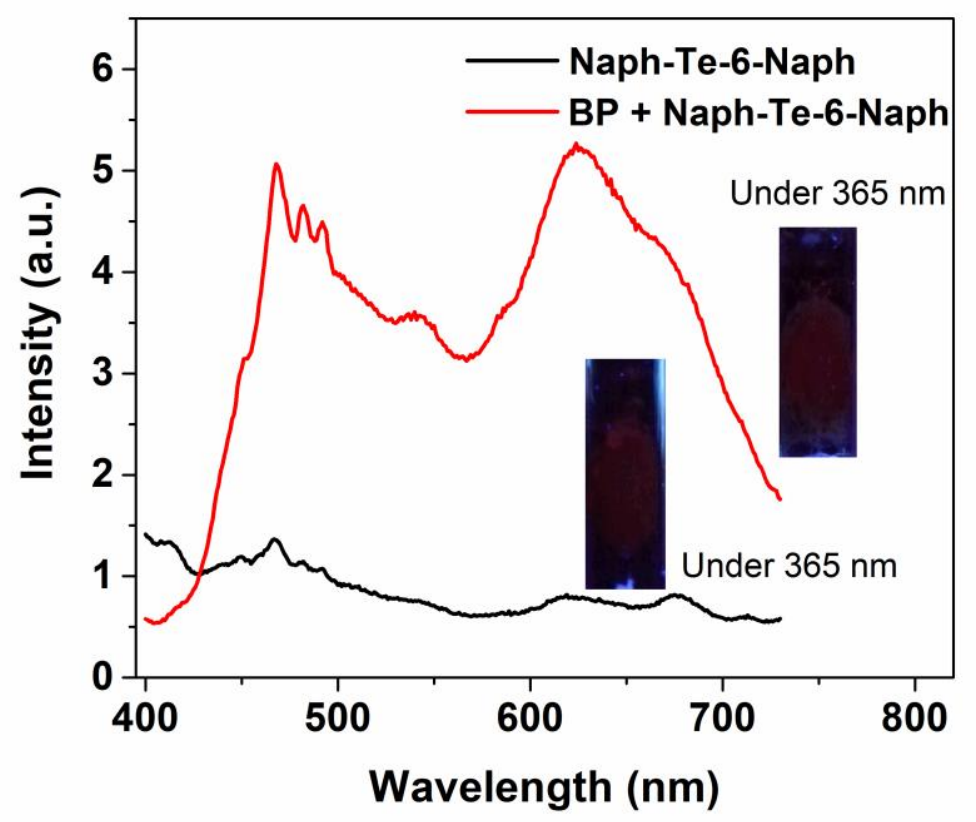

Figure S28. Solid-state emission of Naph-Te-6-Naph and its 1:1 mixture with benzophenone (BP) measured at rt, $\lambda_{\mathrm{ex}}=395 \mathrm{~nm}$. 


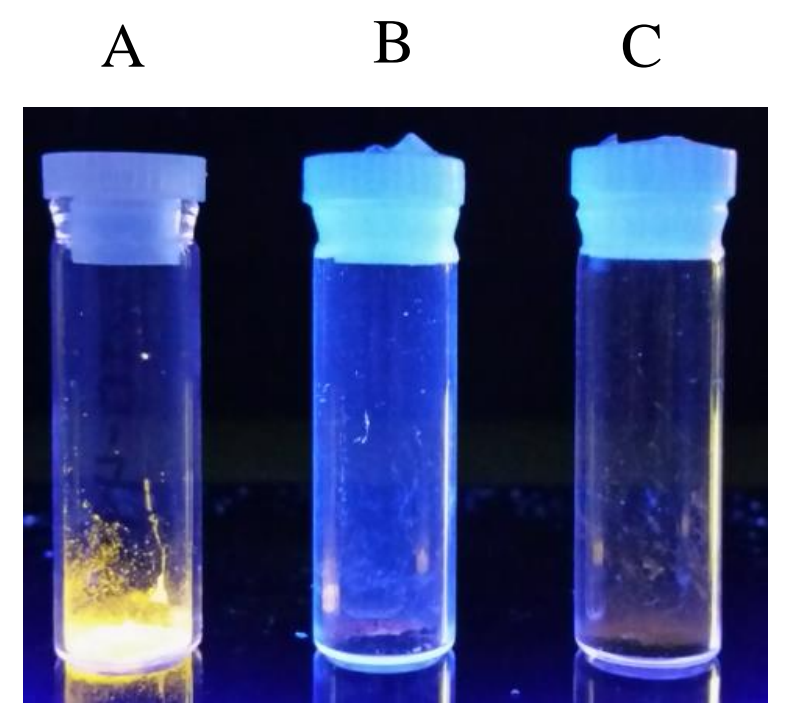

Figure S29. Luminescence in the solid state of A) $\mathbf{A r} \mathbf{r}^{\mathbf{F}}-\mathbf{T e}-\mathbf{6}-\mathbf{A r} \mathbf{r}^{\mathbf{F}}$, B) Napt-Te-6-Naph and C) Fl-Te-6-Fl at room temperature when irradiated at $365 \mathrm{~nm}$.

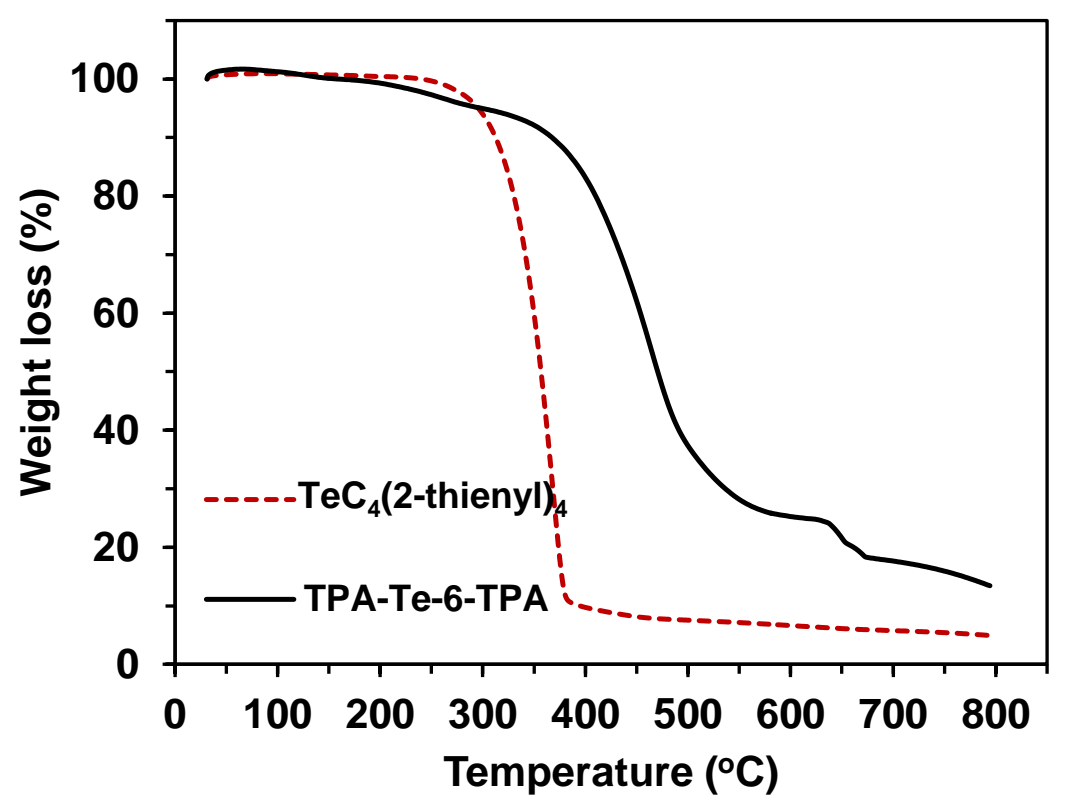

Figure S30. TGA measurements of $\mathrm{TeC}_{4}\left(2\right.$-thienyl) ${ }_{4}$ and TPA-Te-6-TPA. 


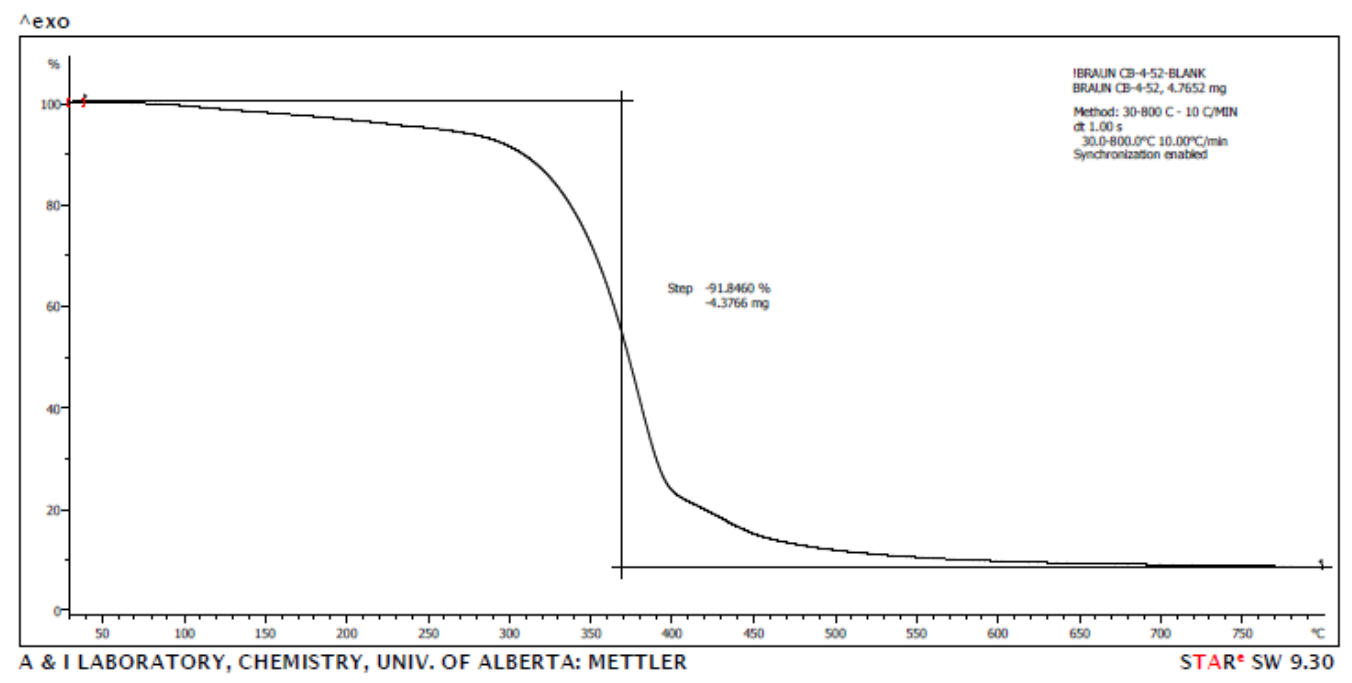

Figure S31. TGA measurements of Benzo-Te(BPin)TPA. 


\section{Crystallographic Data}

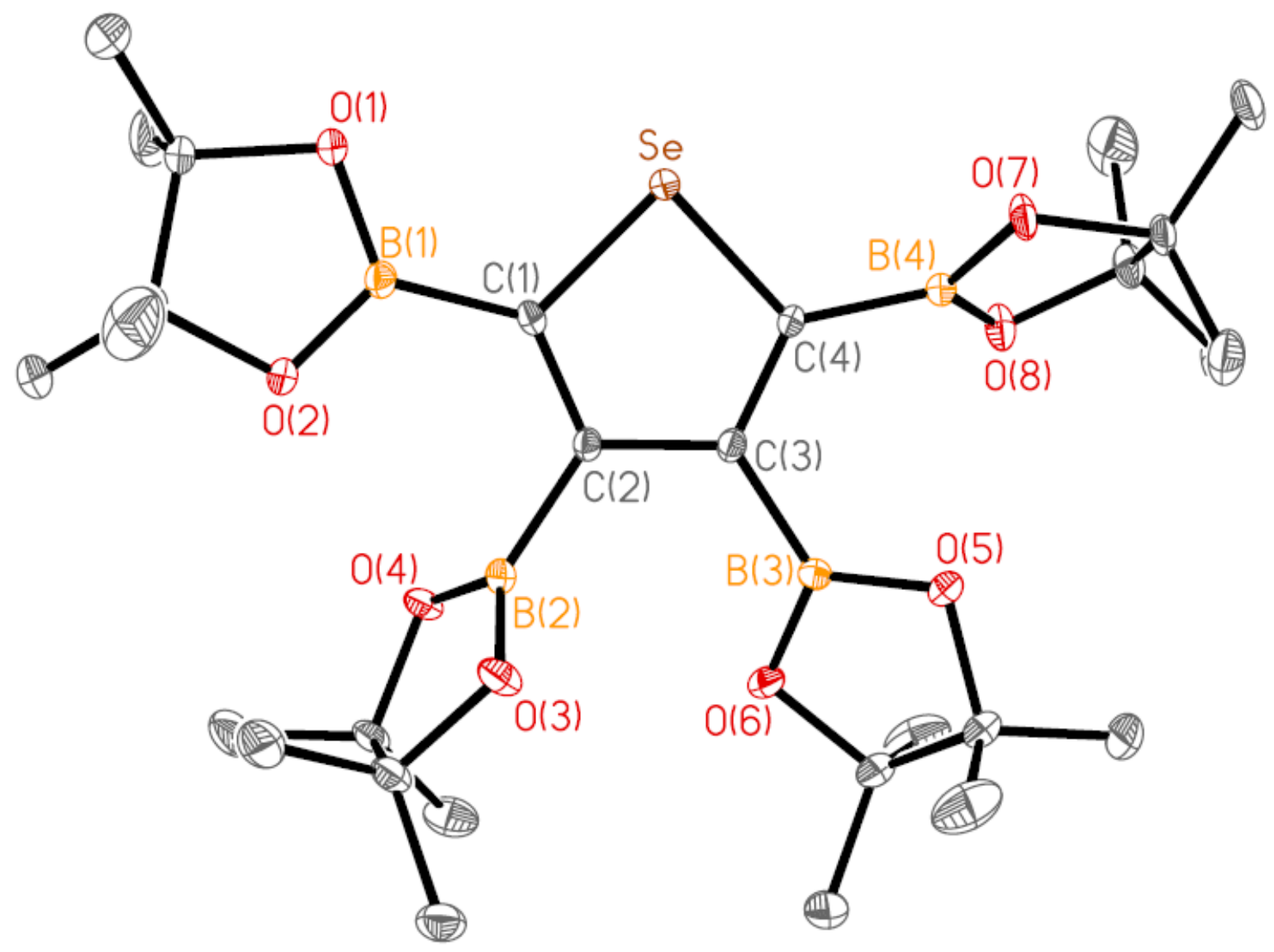

Figure S32. Molecular structure of 4BSe with thermal ellipsoids presented at a $30 \%$ probability level. All hydrogen atoms have been omitted for clarity. Selected bond lengths $(\AA)$ and angles $\left(^{\circ}\right)$ : Se-C(1) 1.876(3), Se-C(4) 1.864(2), C(1)-C(2) 1.374(4), C(2)-C(3) 1.453(3), C(3)-C(4) 1.378(4), C(1)-B(1) 1.544(4), C(2)-B(2) 1.573(4), C(3)-B(3) 1.555(4), C(4)-B(4) 1.561(4); C(1)Se-C(4) 88.5(11), Se-C(1)-C(2) 110.9(17), Se-C(4)-C(3) 110.8(17), C(1)-C(2)-C(3) 114.6(2), $\mathrm{C}(2)-\mathrm{C}(3)-\mathrm{C}(4)$ 115.1(2), $\mathrm{C}(1)-\mathrm{B}(1)-\mathrm{O}(1)$ 124.4(3), $\mathrm{C}(2)-\mathrm{B}(2)-\mathrm{O}(3)$ 124.2(3), $\mathrm{C}(3)-\mathrm{B}(3)-\mathrm{O}(5)$ 123.1(3), C(4)-B(4)-O(7) 121.8(2); O(1)-B(1)-C(1)-Se 1.6(4), O(7)-B(4)-C(4)-Se 60.4(3), C(4)$\mathrm{C}(3)-\mathrm{B}(3)-\mathrm{O}(5)$ 33.0(4), C(1)-C(2)-B(2)-O(4) 70.7(4). 
Table S1. X-Ray Crystallographic Data for Benzo-Te(BPin)TPA (4) and $\mathbf{T e C}_{4}(2$-thienyl) 4 (6) (chloroform solvate).

\begin{tabular}{|l|l|l|}
\hline & 4 & 6 \\
\hline
\end{tabular}

\section{Crystal Data:}

empirical formula

fw

cryst. dimens. $\left(\mathrm{mm}^{3}\right)$

cryst. syst.

space group

unit cell dimensions

$\begin{array}{cc}a(\AA) & 7.58490(10) \\ b(\AA) & 22.1366(4) \\ c(\AA) & 32.3264(5) \\ V\left(\AA^{3}\right) & 5427.73(15) \\ Z & 8 \\ \rho\left(\mathrm{g} \mathrm{cm}^{-3}\right) & 1.466 \\ \text { coeff. }\left(\mathrm{mm}^{-1}\right) & 8.876\end{array}$

\section{Data Collection and Refinement Conditions:}

\begin{tabular}{|c|c|c|}
\hline $\mathrm{T}(\mathrm{K})$ & 173 & $173(1)$ \\
\hline $2 \theta_{\max }(\mathrm{deg})$ & 144.64 & 56.44 \\
\hline total data & 35937 & 20609 \\
\hline unique data $\left(R_{\mathrm{int}}\right)$ & $5351\left(R_{\mathrm{int}}=0.0490\right)$ & $5551\left(R_{\mathrm{int}}=0.0445\right)$ \\
\hline obs. data $\left[F_{o}^{2}>2 \sigma\left(F_{o}^{2}\right)\right]$ & 4763 & 4642 \\
\hline params. & 334 & 292 \\
\hline$R_{1}\left[F_{o}^{2}>2 \sigma\left(F_{o}^{2}\right)\right]^{\mathrm{a}}$ & 0.0388 & 0.0337 \\
\hline$w R_{2}[\text { all data }]^{\mathrm{a}}$ & 0.1142 & 0.0873 \\
\hline $\operatorname{Max} / \operatorname{Min} \Delta \rho\left(\mathrm{e} \AA^{-3}\right)$ & $0.859 /-0.904$ & $1.285 /-0.531$ \\
\hline
\end{tabular}

$$
\begin{gathered}
\mathrm{C}_{21} \mathrm{H}_{13} \mathrm{Cl}_{3} \mathrm{~S}_{4} \mathrm{Te} \\
627.50 \\
0.48 \times 0.09 \times 0.04 \\
\text { orthorhombic }
\end{gathered}
$$$$
\operatorname{Pna2}_{1} \text { (No. 33) }
$$

19.7292 (12)

16.0519 (10)

7.1294 (4)

2257.8 (2)

4

1.846

2.050

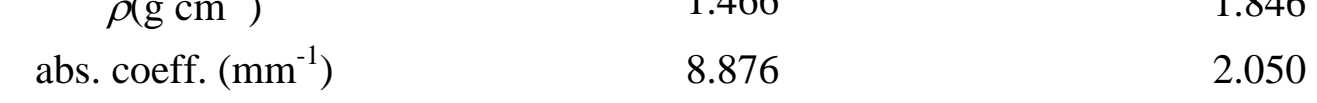


Table S2. X-Ray Crystallographic Data for Naph-Te-6-Naph (7) and $\mathbf{A r}^{\mathrm{F}}-\mathbf{T e}-\mathbf{6}-\mathbf{A r}^{\mathrm{F}}(\mathbf{1 0})$.

7

\section{Crystal Data:}

empirical formula

fw

cryst. dimens. $\left(\mathrm{mm}^{3}\right)$

cryst. syst.

space group

unit cell dimensions

$\begin{array}{lc}a(\AA) & 16.0968(3) \\ b(\AA) & 7.3295(2) \\ c(\AA) & 35.3537(8) \\ \alpha(\mathrm{deg}) & - \\ \beta(\mathrm{deg}) & - \\ \gamma(\mathrm{deg}) & - \\ V\left(\AA^{3}\right) & 4171.08(17) \\ Z & 8 \\ \rho\left(\mathrm{g} \mathrm{cm}^{-3}\right) & 1.548 \\ \operatorname{coeff.~}\left(\mathrm{mm}^{-1}\right) & 11.32\end{array}$

$0.20 \times 0.17 \times 0.04$

orthorhombic

Pbca (No. 61)
10

$$
\begin{gathered}
\mathrm{C}_{24} \mathrm{H}_{14} \mathrm{~F}_{12} \mathrm{Te} \\
657.95
\end{gathered}
$$

$0.42 \times 0.24 \times 0.08$

triclinic

$P \overline{1}$ (No. 2)

$11.6764(8)$

$14.6438(11)$

$15.8273(11)$

$68.5869(8)$

$72.8467(8)$

$73.0843(8)$

$2355.7(3)$

4

1.855

1.369

\section{Data Collection and Refinement Conditions:}

$\begin{array}{ccc}\mathrm{T}(\mathrm{K}) & 173 & 173 \\ 2 \Theta_{\max }(\mathrm{deg}) & 148.03 & 56.48 \\ \text { total data } & 23232 & 22229 \\ \text { unique data }\left(R_{\mathrm{int}}\right) & 4108\left(R_{\mathrm{int}}=0.0673\right) & 11350\left(R_{\mathrm{int}}=0.0173\right) \\ \text { obs. data }\left[F_{o}^{2} \geq 2 \sigma\left(F_{o}^{2}\right)\right] & 3772 & 9459 \\ \text { params. } & 262 & 667 \\ R_{1}\left[F_{o}^{2} \geq 2 \sigma\left(F_{o}^{2}\right)\right]^{\mathrm{a}} & 0.0653 & 0.0387 \\ w R_{2}[\text { all data }]^{\mathrm{a}} & 0.1463 & 0.1051 \\ \operatorname{Max} / \mathrm{Min} \Delta \rho\left(\mathrm{e} \AA^{-3}\right) & 1.208 /-2.466 & 1.428 /-0.768 \\ { }^{a} R_{1}=\Sigma\left\|F_{\mathrm{o}}|-| F_{\mathrm{c}}\right\| / \Sigma\left|F_{\mathrm{o}}\right| ; w R_{2}=\left[\Sigma w\left(F_{\mathrm{o}}{ }^{2}-F_{\mathrm{c}}{ }^{2}\right)^{2} / \Sigma w\left(F_{\mathrm{o}}{ }^{4}\right)\right]^{1 / 2} & \end{array}$


Table S3. X-Ray Crystallographic Data for 4BSe.

\begin{tabular}{|c|c|}
\hline & 4BSe \\
\hline
\end{tabular}

\section{Crystal Data:}

empirical formula

fw

$\mathrm{C}_{28} \mathrm{H}_{48} \mathrm{~B}_{4} \mathrm{O}_{8} \mathrm{Se}$

634.86

cryst. dimens. $\left(\mathrm{mm}^{3}\right)$

cryst. syst.

space group

$0.40 \times 0.31 \times 0.30$

monoclinic

$P 2_{1}$ (No. 4)

unit cell dimensions

$\begin{array}{cc}a(\AA) & 10.4690(11) \\ b(\AA) & 13.8325(14) \\ c(\AA) & 11.9952(12) \\ \beta(\mathrm{deg}) & 100.7232(11) \\ V\left(\AA^{3}\right) & 1706.7(3) \\ Z & 2 \\ \rho\left(\mathrm{g} \mathrm{cm}^{-3}\right) & 1.235\end{array}$

abs. coeff. $\left(\mathrm{mm}^{-1}\right) \quad 1.142$

\section{Data Collection and Refinement Conditions:}

\begin{tabular}{cc}
$\mathrm{T}(\mathrm{K})$ & 173 \\
$2 \theta_{\max }(\mathrm{deg})$ & 55.16 \\
total data & 39904 \\
unique data $\left(R_{\mathrm{int}}\right)$ & $7793\left(R_{\text {int }}=0.0306\right)$ \\
obs. data $\left[F_{\mathrm{O}} 2 \geq 2 \sigma\left(F_{\mathrm{O}}{ }^{2}\right)\right]$ & 7534 \\
params. & 371 \\
$R_{1}\left[F_{\mathrm{O}}{ }^{2} \geq 2 \sigma\left(F_{\mathrm{O}}{ }^{2}\right)\right]^{\mathrm{a}}$ & 0.0264 \\
$w R_{2}[\text { all data }]^{\mathrm{a}}$ & 0.0668 \\
$\operatorname{Max} / \mathrm{Min} \Delta \rho\left(\mathrm{e} \AA^{-3}\right)$ & $0.490 /-0.791$ \\
\hline${ }^{a} R_{1}=\Sigma \| F_{\mathrm{o}}|-| F_{\mathrm{c}}|/ \Sigma| F_{\mathrm{o}} \mid ; w R_{2}=\left[\Sigma w\left(F_{\mathrm{o}}{ }^{2}-F_{\mathrm{c}}{ }^{2}\right)^{2} / \Sigma w\left(F_{\mathrm{o}}{ }^{4}\right)\right]^{1 / 2}$.
\end{tabular}




\section{Computational Results}

\subsection{Computational Methods}

Ground state geometry optimizations were performed in the gas-phase using density functional theory (DFT) with the hybrid B3LYP ${ }^{\mathrm{S} 1, \mathrm{~S} 2}$ functional and the cc-pVTZ/cc-pVTZ-PP(Te) basis sets; ${ }^{\mathrm{S} 3-\mathrm{S} 5}$ hereafter referred to as cc-pVTZ-(PP). The basis sets and corresponding effective core potential (ECP) for tellurium were obtained from the Basis Set Exchange library. ${ }^{\text {S6, S7 }}$ Using the same level of theory, harmonic vibrational frequencies were computed to confirm the optimized geometries as minima on the potential energy surfaces. In the present work, the absorption (vertical excitation) energies were predicted using time-dependent density functional theory (TDDFT) for the lowest three singlet and triplet states; the TD-DFT computations were undertaken at the B3LYP/cc-pVTZ-(PP) level of theory. Previous work ${ }^{\mathrm{S} 8}$ on tellurophene containing systems explored the choice of functional, basis set, and inclusion of solvent effects on the vertical excitation energies as determined using TD-DFT; these benchmark results demonstrated that the present choice (B3LYP/cc-pVTZ-(PP)) is suitable for interpreting the excitation and potential coupling between the singlet and triplet states (at least via energy gap considerations). For a limited number of compounds, where charge-transfer may play a role as indicated by the $\Lambda$-diagnostic parameter, ${ }^{\mathrm{S} 9}$ the vertical excitation energies were computed using the hybrid metaGGG functional M06-2X ${ }^{\mathrm{S} 10}$ and the range-separated hybrid functional CAM-B3LYP.S11 ${ }^{\text {The }}$ $\Lambda$-diagnostic parameter was determined using the GabEdit software package. ${ }^{\text {S12 }}$ These computations used the same cc-pVTZ/cc-pVTZ-PP(Te) basis sets. ${ }^{\text {S3-S5 }}$ For some compounds, the role of solvation on the vertical excitation energies was examined using the polarizable continuum model (IEF-PCM ${ }^{\mathrm{S} 13, \mathrm{~S} 14}$ and universal force field (UFF) atomic radii) with parameters for THF. All of the present electronic structure computations were carried out using Gaussian09. ${ }^{\text {S15 }}$

For the tellurophenes, there is interest in both the absorption (corresponding to $S_{0}$ to $S_{1}$ excitation) and phosphorescence (estimated as $T_{1}$ to $S_{0}$ energy difference) energies. In the present work, and as mentioned above, the absorption (vertical excitation) energies were predicted using time-dependent density functional theory (TD-DFT) for the lowest three singlet and triplet states. For obtaining the phosphorescence energies, the lowest energy triplet state was optimized at the UB3LYP/ cc-pVTZ - (PP) level of theory. The phosphorescence energies can be 
estimated from (i) the singlet to triplet energy gap at the optimized geometry for the triplet state ( $E_{\text {vertical }}$; (ii) the energy difference between the lowest energy triplet and ground state singlet at their corresponding optimized geometries $\left(\mathrm{E}_{\text {adiabatic }}\right)$; or (iii) the energy difference between the lowest energy triplet and ground state singlet at their corresponding optimized geometries including the zero-point vibrational energy difference $\left(\mathrm{E}_{0-0}\right)$. In previous computations on tellurophene systems, ${ }^{\mathrm{S} 8 \mathrm{~S} 16}$ the phosphorescence energies are under-estimated from the $\mathrm{T}_{1}$ (UB3LYP) to $\mathrm{S}_{0}$ (B3LYP) values given by method (i); similar under-estimation comes from TDB3LYP results carried out at the $\mathrm{T}_{1}$ geometry.

Table S4. The excitation energies ${ }^{\mathrm{a}}$ (in $\mathrm{eV}$ ), absorption wavelengths (nm), and oscillator strengths (f) of the lowest ten lying singlet and ten lying triplet states for TPA-Te-6-TPA at the TDB3LYP/cc-pVTZ-(PP) level of theory at the gas-phase $\mathrm{S}_{0}$ geometry (as determined at the B3LYP/cc-pVTZ-(PP) level of theory).

\begin{tabular}{cccc}
\multirow{2}{*}{ Excited state } & \multicolumn{3}{c}{ TPA-Te-6-TPA } \\
\cline { 2 - 4 } & $\mathrm{E} / \mathrm{eV}$ & $\lambda / \mathrm{nm}$ & $\mathrm{f}$ \\
\hline $\mathbf{T}_{\mathbf{1}}$ & 2.4341 & 509.35 & 0.0000 \\
$\mathbf{T}_{\mathbf{2}}$ & 2.8342 & 437.46 & 0.0000 \\
$\mathbf{T}_{\mathbf{3}}$ & 3.1381 & 395.09 & 0.0000 \\
$\mathbf{T}_{\mathbf{4}}$ & 3.1485 & 393.79 & 0.0000 \\
$\mathbf{T}_{\mathbf{5}}$ & 3.1516 & 393.40 & 0.0000 \\
$\mathbf{T}_{\mathbf{6}}$ & 3.1627 & 392.02 & 0.0000 \\
$\mathbf{S}_{\mathbf{1}}$ & 3.1801 & 389.87 & 1.2055 \\
$\mathbf{T}_{\mathbf{7}}$ & 3.2516 & 381.30 & 0.0000 \\
$\mathbf{S}_{\mathbf{2}}$ & 3.2681 & 379.38 & 0.0108 \\
$\mathbf{T}_{\mathbf{8}}$ & 3.2695 & 379.21 & 0.0000 \\
$\mathbf{T}_{\mathbf{9}}$ & 3.2772 & 378.33 & 0.0000 \\
$\mathbf{T}_{\mathbf{1 0}}$ & 3.3902 & 365.72 & 0.0000 \\
$\mathbf{S}_{\mathbf{3}}$ & 3.5308 & 351.15 & 0.0126 \\
$\mathbf{S}_{\mathbf{4}}$ & 3.5652 & 347.77 & 0.0312 \\
$\mathbf{S}_{\mathbf{5}}$ & 3.6397 & 340.64 & 0.0244 \\
$\mathbf{S}_{\mathbf{6}}$ & 3.6541 & 339.30 & 0.0036 \\
$\mathbf{S}_{\mathbf{7}}$ & 3.7536 & 330.31 & 0.0374 \\
$\mathbf{S}_{\mathbf{8}}$ & 3.7664 & 329.19 & 0.0013 \\
$\mathbf{S}_{\mathbf{9}}$ & 3.8761 & 319.87 & 0.1545 \\
$\mathbf{S}_{\mathbf{1 0}}$ & 3.8806 & 319.50 & 0.1950
\end{tabular}

a) Energies (wavelengths) reported to 4 (2) decimal places to facilitate present and future computational comparison. 


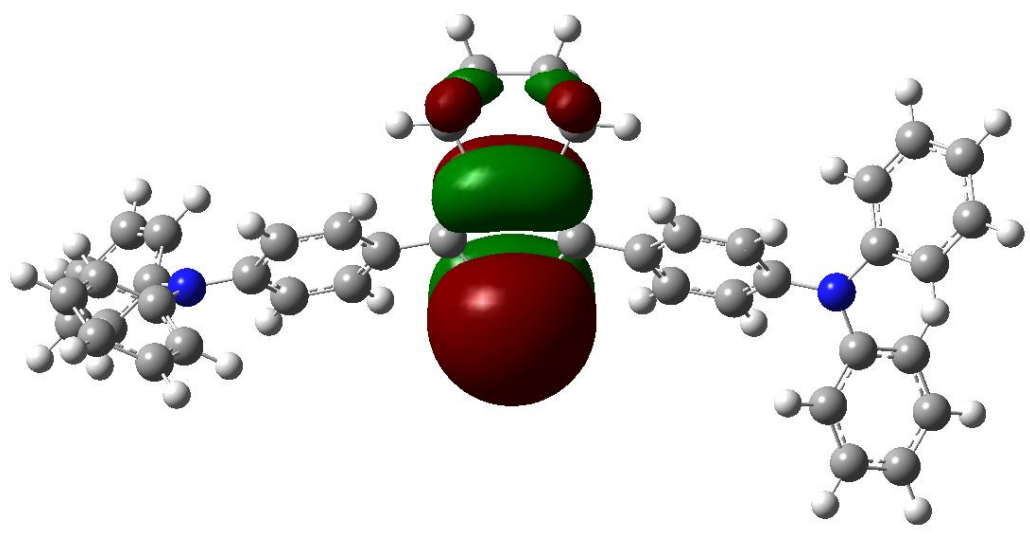

(a) HOMO-2

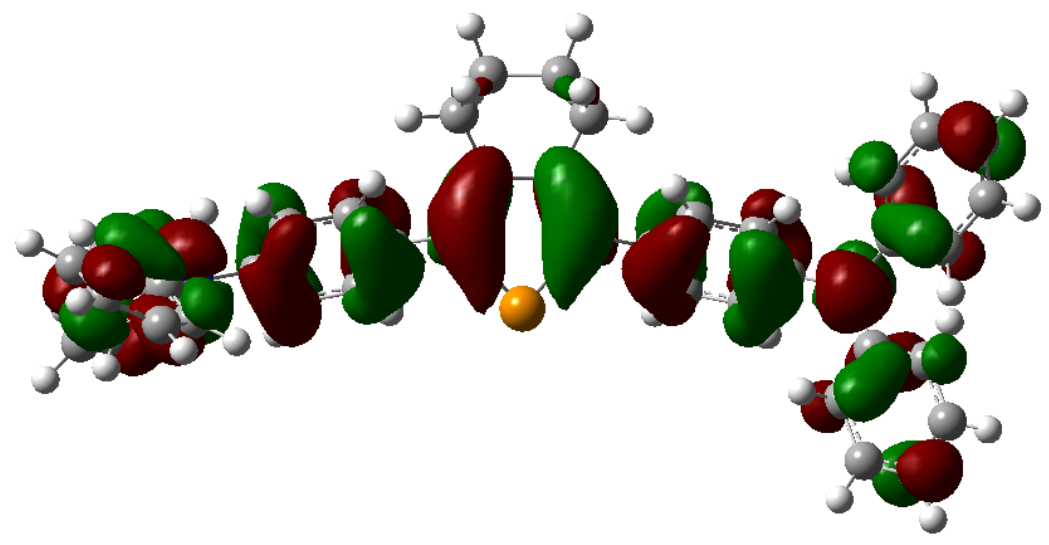

(b) HOMO

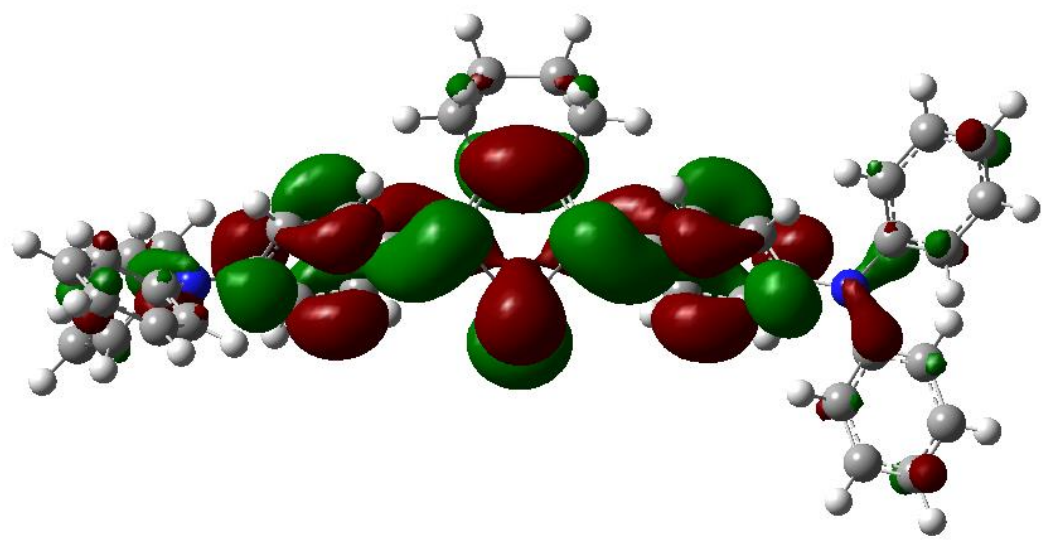

(c) LUMO

Figure S33. a) HOMO-2, b) HOMO, and c) LUMO for TPA-Te-6-TPA at the TD-B3LYP/ccpVTZ-(PP) level of theory at the gas-phase $\mathrm{S}_{0}$ geometry (as determined at the B3LYP/cc-pVTZ(PP) level of theory). Excitation to $\mathrm{S}_{1}$ involves HOMO $\rightarrow$ LUMO $(\mathrm{C}=0.69466)$. Excitation to $\mathrm{S}_{8}$ involves HOMO-2 $\rightarrow$ LUMO $(\mathrm{C}=0.69595,3.5308 \mathrm{eV}, 351.15 \mathrm{~nm}, \mathrm{f}=0.0126)$. All orbitals illustrated with an isovalue $=0.02$. 
Table S5. Comparison of selected bond lengths $(\AA)$ and angles $\left(^{\circ}\right)$ for Benzo-Te(BPin)TPA determined from the experimental X-ray structure and those computed at the B3LYP/cc-pVTZ(PP) level of theory in the gas-phase. Atom labels are provided in Figure 2 in the main text.

\begin{tabular}{|c|c|c|}
\hline Parameter & Experiment & Theory \\
\hline Te-C(1) & $2.079(3)$ & 2.10613 \\
\hline Te-C(8) & $2.058(4)$ & 2.08941 \\
\hline $\mathrm{C}(1)-\mathrm{C}(2)$ & $1.361(4)$ & 1.36469 \\
\hline $\mathrm{C}(2)-\mathrm{C}(3)$ & $1.464(4)$ & 1.45744 \\
\hline $\mathrm{C}(8)-\mathrm{C}(3)$ & $1.411(4)$ & 1.41286 \\
\hline $\mathrm{C}(8)-\mathrm{Te}-\mathrm{C}(1)$ & $82.07(13)$ & 81.764 \\
\hline $\mathrm{Te}-\mathrm{C}(1)-\mathrm{B}$ & $117.7(2)$ & 116.623 \\
\hline $\mathrm{Te}-\mathrm{C}(1)-\mathrm{C}(2)$ & $112.4(2)$ & 111.854 \\
\hline $\mathrm{C}(1)-\mathrm{C}(2)-\mathrm{C}(3)$ & $117.5(3)$ & 118.118 \\
\hline $\mathrm{C}(2)-\mathrm{C}(3)-\mathrm{C}(8)$ & $116.2(3)$ & 117.144 \\
\hline $\mathrm{C}(3)-\mathrm{C}(8)-\mathrm{Te}$ & $111.8(2)$ & 111.117 \\
\hline $\mathrm{Te}-\mathrm{C}(1)-\mathrm{B}-\mathrm{O}(1)$ & 180.0 & 175.6 \\
\hline $\mathrm{Tilt}-\mathrm{TPA}$ & $51.9(4)$ & 67.4 \\
\hline
\end{tabular}

Table S6. $\Lambda$-diagnostic for the $S_{1}$ and $S_{2}$ states for Benzo-Te(BPin)TPA at the TD-B3LYP/ccpVTZ-(PP) level of theory in the gas-phase as well as TD-CAM-B3LYP/cc-pVTZ-(PP) and TDM06-2X/cc-pVTZ-(PP) levels of theory in THF. All computations at the gas-phase $\mathrm{S}_{0}$ geometry (as determined at the B3LYP/cc-pVTZ-(PP) level of theory). Values less than 0.4 suggest charge-transfer or long-range character to the excitation.

\begin{tabular}{|l|c|c|c|}
\hline State & B3LYP & CAM-B3LYP & M06-2X \\
\hline $\mathbf{S}_{\mathbf{1}}$ & 0.388 & 0.539 & 0.542 \\
\hline $\mathbf{S}_{\mathbf{2}}$ & 0.622 & 0.547 & 0.517 \\
\hline
\end{tabular}


Table S7. Excitation energies ${ }^{\mathrm{a}}$ (in $\mathrm{eV}$ ), absorption wavelengths $(\mathrm{nm}$ ), and oscillator strengths (f) of the ten lowest-lying singlet and triplet states for Benzo-Te(BPin)TPA at the TD-B3LYP/ccpVTZ-(PP), TD-CAM-B3LYP/cc-pVTZ-(PP), and TD-M06-2X/cc-pVTZ-(PP) levels of theory in the gas-phase. All computations at the gas-phase $\mathrm{S}_{0}$ geometry (as determined at the B3LYP/cc-pVTZ-(PP) level of theory).

\begin{tabular}{cccccccccccc} 
& \multicolumn{3}{c}{ TD-B3LYP } & \multicolumn{4}{c}{ TD-CAM-B3LYP } & \multicolumn{3}{c}{ TD-M06-2X } \\
\cline { 2 - 12 } State & $\mathrm{E} / \mathrm{eV}$ & $\lambda / \mathrm{nm}$ & $\mathrm{f}$ & State & $\mathrm{E} / \mathrm{eV}$ & $\lambda / \mathrm{nm}$ & $\mathrm{f}$ & State & $\mathrm{E} / \mathrm{eV}$ & $\lambda / \mathrm{nm}$ & $\mathrm{f}$ \\
\hline $\mathrm{T}_{1}$ & 2.5750 & 481.50 & 0.0000 & $\mathrm{~T}_{1}$ & 2.5756 & 481.39 & 0.0000 & $\mathrm{~T}_{1}$ & 2.9606 & 418.78 & 0.0000 \\
$\mathrm{~T}_{2}$ & 3.0109 & 411.79 & 0.0000 & $\mathrm{~T}_{2}$ & 3.0832 & 402.13 & 0.0000 & $\mathrm{~T}_{2}$ & 3.2522 & 381.23 & 0.0000 \\
$\mathrm{~T}_{3}$ & 3.1556 & 392.90 & 0.0000 & $\mathrm{~T}_{3}$ & 3.1553 & 392.94 & 0.0000 & $\mathrm{~T}_{3}$ & 3.4880 & 355.46 & 0.0000 \\
$\mathrm{~T}_{4}$ & 3.2027 & 387.12 & 0.0000 & $\mathrm{~T}_{4}$ & 3.2414 & 382.50 & 0.0000 & $\mathrm{~T}_{4}$ & 3.5853 & 345.81 & 0.0000 \\
$\mathrm{~S}_{1}$ & 3.2110 & 386.12 & 0.1264 & $\mathrm{~T}_{5}$ & 3.3515 & 369.94 & 0.0000 & $\mathrm{~T}_{5}$ & 3.6364 & 340.95 & 0.0000 \\
$\mathrm{~T}_{5}$ & 3.2626 & 380.02 & 0.0000 & $\mathrm{~T}_{6}$ & 3.5881 & 345.54 & 0.0000 & $\mathrm{~S}_{1}$ & 3.6899 & 336.01 & 0.0143 \\
$\mathrm{~T}_{6}$ & 3.3365 & 371.59 & 0.0000 & $\mathrm{~S}_{1}$ & 3.7033 & 334.79 & 0.0163 & $\mathrm{~T}_{6}$ & 3.8112 & 325.31 & 0.0000 \\
$\mathrm{~T}_{7}$ & 3.3934 & 365.36 & 0.0000 & $\mathrm{~T}_{7}$ & 3.7296 & 332.43 & 0.0000 & $\mathrm{~S}_{2}$ & 3.8905 & 318.68 & 0.2440 \\
$\mathrm{~S}_{2}$ & 3.5427 & 349.97 & 0.0577 & $\mathrm{~S}_{2}$ & 3.8638 & 320.89 & 0.2206 & $\mathrm{~T}_{7}$ & 4.1515 & 298.65 & 0.0000 \\
$\mathrm{~S}_{3}$ & 3.5958 & 344.81 & 0.0066 & $\mathrm{~T}_{8}$ & 3.8679 & 320.55 & 0.0000 & $\mathrm{~S}_{3}$ & 4.1737 & 297.06 & 0.1049
\end{tabular}

a) Energies (wavelengths) reported to 4 (2) decimal places to facilitate present and future computational comparison.

Table S8. Excitation energies ${ }^{\mathrm{a}}$ (in $\mathrm{eV}$ ), absorption wavelengths (nm), and oscillator strengths (f) of the ten lowest-lying singlet and triplet states for Benzo-Te(BPin)TPA at the TD-B3LYP/ccpVTZ-(PP), TD-CAM-B3LYP/cc-pVTZ-(PP), and TD-M06-2X/cc-pVTZ-(PP) levels of theory in THF (PCM). All computations at the gas-phase $\mathrm{S}_{0}$ geometry (as determined at the $\mathrm{B} 3 \mathrm{LYP} / \mathrm{cc}-$ pVTZ-(PP) level of theory).

\begin{tabular}{cccccccccccc} 
& \multicolumn{3}{c}{ TD-B3LYP } & \multicolumn{4}{c}{ TD-CAM-B3LYP } & \multicolumn{3}{c}{ TD-M06-2X } \\
\cline { 2 - 11 } State & $\mathrm{E} / \mathrm{eV}$ & $\lambda / \mathrm{nm}$ & $\mathrm{f}$ & & $\mathrm{E} / \mathrm{eV}$ & $\lambda / \mathrm{nm}$ & $\mathrm{f}$ & & $\mathrm{E} / \mathrm{eV}$ & $\lambda / \mathrm{nm}$ & $\mathrm{f}$ \\
\hline $\mathrm{T}_{1}$ & 2.5968 & 477.46 & 0.0000 & $\mathrm{~T}_{1}$ & 2.5943 & 477.91 & 0.0000 & $\mathrm{~T}_{1}$ & 2.9860 & 415.22 & 0.0000 \\
$\mathrm{~T}_{2}$ & 2.9976 & 413.62 & 0.0000 & $\mathrm{~T}_{2}$ & 3.0874 & 401.58 & 0.0000 & $\mathrm{~T}_{2}$ & 3.3297 & 372.3 & 0.0000 \\
$\mathrm{~S}_{1}$ & 3.1531 & 393.21 & 0.1515 & $\mathrm{~T}_{3}$ & 3.1712 & 390.97 & 0.0000 & $\mathrm{~T}_{3}$ & 3.4835 & 355.92 & 0.0000 \\
$\mathrm{~T}_{3}$ & 3.1719 & 390.89 & 0.0000 & $\mathrm{~T}_{4}$ & 3.3169 & 373.80 & 0.0000 & $\mathrm{~T}_{4}$ & 3.6039 & 344.03 & 0.0000 \\
$\mathrm{~T}_{4}$ & 3.2559 & 380.80 & 0.0000 & $\mathrm{~T}_{5}$ & 3.3898 & 365.75 & 0.0000 & $\mathrm{~T}_{5}$ & 3.6672 & 338.09 & 0.0000 \\
$\mathrm{~T}_{5}$ & 3.2906 & 376.78 & 0.0000 & $\mathrm{~T}_{6}$ & 3.6012 & 344.29 & 0.0000 & $\mathrm{~S}_{1}$ & 3.7496 & 330.66 & 0.0431 \\
$\mathrm{~T}_{6}$ & 3.3312 & 372.19 & 0.0000 & $\mathrm{~T}_{7}$ & 3.7365 & 331.82 & 0.0000 & $\mathrm{~T}_{6}$ & 3.8177 & 324.76 & 0.0000 \\
$\mathrm{~T}_{7}$ & 3.3826 & 366.54 & 0.0000 & $\mathrm{~S}_{1}$ & 3.7598 & 329.76 & 0.0532 & $\mathrm{~S}_{2}$ & 3.8766 & 319.83 & 0.3375 \\
$\mathrm{~S}_{2}$ & 3.5531 & 348.95 & 0.1019 & $\mathrm{~S}_{2}$ & 3.8589 & 321.29 & 0.2970 & $\mathrm{~S}_{3}$ & 4.1410 & 299.40 & 0.1279 \\
$\mathrm{~S}_{3}$ & 3.6276 & 341.78 & 0.0298 & $\mathrm{~T}_{8}$ & 3.8914 & 318.61 & 0.0000 & $\mathrm{~T}_{7}$ & 4.1603 & 298.01 & 0.0000
\end{tabular}

a) Energies (wavelengths) reported to 4 (2) decimal places to facilitate present and future computational comparison. 
Table S9. Nature of the lowest-lying singlet states $\left(S_{1}, S_{2}\right.$, and $\left.S_{3}\right)$ for Benzo-Te(BPin)TPA at the TD-B3LYP/cc-pVTZ-(PP), TD-CAM-B3LYP/cc-pVTZ-(PP), and TD-M06-2X/cc-pVTZ(PP) levels of theory in the gas-phase and THF (PCM). All computations at the gas-phase $\mathrm{S}_{0}$ geometry (as determined at the B3LYP/cc-pVTZ-(PP) level of theory). HOMO = Orbital 137 and LUMO = Orbital 138.

\begin{tabular}{|c|c|c|c|c|c|c|}
\hline \multirow{2}{*}{$\begin{array}{l}\text { State } \\
\text { Gas-ph }\end{array}$} & \multicolumn{2}{|l|}{ TD-B3LYP } & \multicolumn{2}{|c|}{ TD-CAM-B3LYP } & \multicolumn{2}{|l|}{ TD-M062X } \\
\hline & & & & & & \\
\hline \multirow[t]{6}{*}{$S_{1}$} & $137 \rightarrow 138$ & 0.70172 & $136 \rightarrow 138$ & -0.15298 & $136 \rightarrow 138$ & -0.13685 \\
\hline & & & $136 \rightarrow 139$ & 0.52526 & $136 \rightarrow 139$ & 0.49828 \\
\hline & & & $136 \rightarrow 142$ & -0.28338 & $136 \rightarrow 140$ & 0.12179 \\
\hline & & & $137 \rightarrow 138$ & -0.10158 & $136 \rightarrow 142$ & 0.31897 \\
\hline & & & $137 \rightarrow 139$ & 0.25386 & $137 \rightarrow 139$ & 0.24288 \\
\hline & & & & & $137 \rightarrow 142$ & 0.11180 \\
\hline \multirow[t]{4}{*}{$\mathrm{S}_{2}$} & $136 \rightarrow 138$ & 0.60369 & $136 \rightarrow 138$ & 0.52055 & $136 \rightarrow 138$ & 0.48074 \\
\hline & $136 \rightarrow 139$ & -0.26462 & $136 \rightarrow 139$ & 0.19146 & $136 \rightarrow 139$ & 0.17700 \\
\hline & $136 \rightarrow 142$ & 0.11877 & $137 \rightarrow 138$ & 0.39986 & $137 \rightarrow 138$ & 0.45277 \\
\hline & $137 \rightarrow 139$ & -0.18152 & & & $137 \rightarrow 142$ & 0.10281 \\
\hline \multirow[t]{7}{*}{$\mathrm{S}_{3}$} & $136 \rightarrow 138$ & 0.32830 & $136 \rightarrow 138$ & -0.25733 & $136 \rightarrow 138$ & 0.37207 \\
\hline & $136 \rightarrow 139$ & 0.44250 & $136 \rightarrow 140$ & -0.12232 & $137 \rightarrow 138$ & -0.33145 \\
\hline & $136 \rightarrow 142$ & -0.13292 & $137 \rightarrow 138$ & 0.28442 & $137 \rightarrow 139$ & 0.27575 \\
\hline & $137 \rightarrow 139$ & 0.39999 & $137 \rightarrow 139$ & -0.21836 & $137 \rightarrow 140$ & -0.28960 \\
\hline & & & $137 \rightarrow 140$ & 0.44898 & $137 \rightarrow 142$ & -0.17225 \\
\hline & & & $137 \rightarrow 142$ & -0.15985 & & \\
\hline & & & $137 \rightarrow 143$ & -0.10143 & & \\
\hline \multicolumn{7}{|c|}{ In THF } \\
\hline \multirow[t]{6}{*}{$\mathrm{S}_{1}$} & $137 \rightarrow 138$ & 0.70140 & $136 \rightarrow 138$ & -0.22161 & $136 \rightarrow 138$ & -0.18456 \\
\hline & & & $136 \rightarrow 139$ & 0.51365 & $136 \rightarrow 139$ & 0.49418 \\
\hline & & & $136 \rightarrow 142$ & -0.27715 & $136 \rightarrow 140$ & 0.10006 \\
\hline & & & $137 \rightarrow 138$ & 0.14712 & $136 \rightarrow 142$ & 0.31790 \\
\hline & & & $137 \rightarrow 139$ & -0.22019 & $137 \rightarrow 138$ & 0.13789 \\
\hline & & & & & $137 \rightarrow 139$ & -0.21899 \\
\hline \multirow[t]{4}{*}{$\mathrm{S}_{2}$} & $136 \rightarrow 138$ & 0.66902 & $136 \rightarrow 138$ & 0.50297 & $136 \rightarrow 138$ & 0.45804 \\
\hline & $136 \rightarrow 139$ & -0.13694 & $136 \rightarrow 139$ & 0.26382 & $136 \rightarrow 139$ & 0.23011 \\
\hline & & & $137 \rightarrow 138$ & -0.37227 & $137 \rightarrow 138$ & -0.44220 \\
\hline & & & $137 \rightarrow 142$ & 0.11450 & $137 \rightarrow 142$ & -0.12781 \\
\hline \multirow[t]{6}{*}{$\mathrm{S}_{3}$} & $136 \rightarrow 138$ & -0.16528 & $136 \rightarrow 138$ & 0.32928 & $136 \rightarrow 138$ & 0.42321 \\
\hline & $136 \rightarrow 139$ & -0.46643 & $137 \rightarrow 138$ & 0.34665 & $137 \rightarrow 138$ & 0.34002 \\
\hline & $136 \rightarrow 142$ & 0.14874 & $137 \rightarrow 139$ & -0.25219 & $137 \rightarrow 139$ & -0.27559 \\
\hline & $137 \rightarrow 139$ & 0.46870 & $137 \rightarrow 140$ & 0.28431 & $137 \rightarrow 140$ & 0.18112 \\
\hline & & & $137 \rightarrow 142$ & -0.22404 & $137 \rightarrow 142$ & 0.18782 \\
\hline & & & $137 \rightarrow 143$ & -0.14891 & $137 \rightarrow 143$ & -0.13241 \\
\hline
\end{tabular}




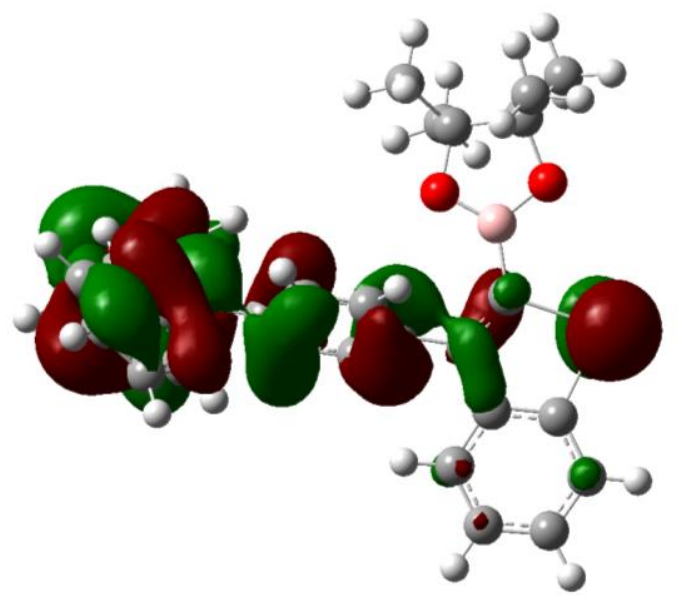

(a) HOMO

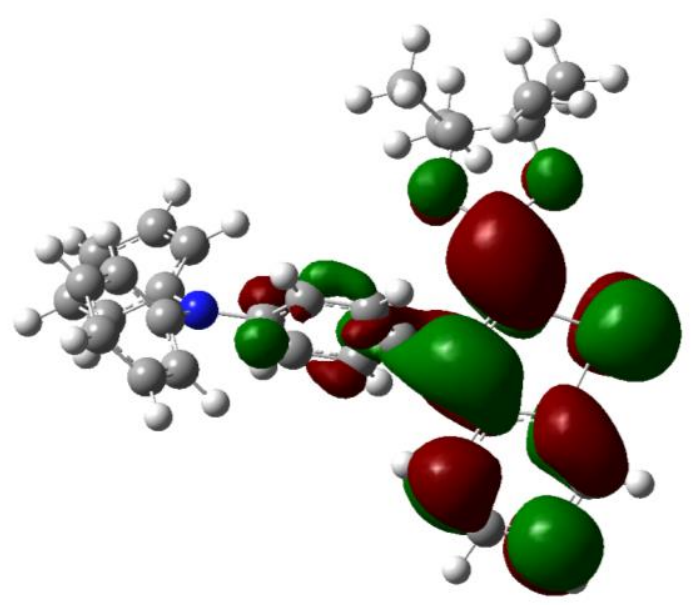

(b) LUMO

Figure S34. a) HOMO and b) LUMO corresponding to the lowest excitation to $S_{1}$ for BenzoTe(BPin)TPA at the TD-B3LYP/cc-pVTZ-(PP) level of theory at the gas-phase $\mathrm{S}_{0}$ geometry (as determined at the B3LYP/cc-pVTZ-(PP) level of theory). All orbitals illustrated with an isovalue $=0.02$. 


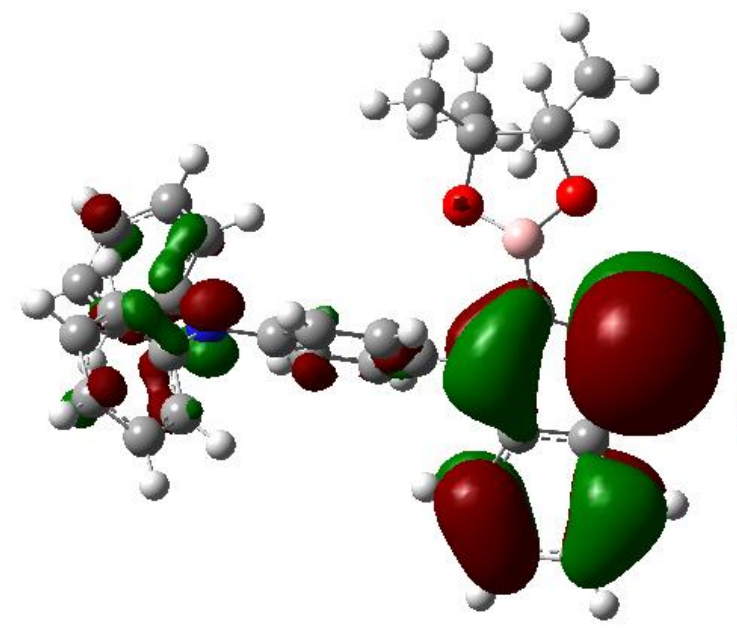

(a) HOMO-1

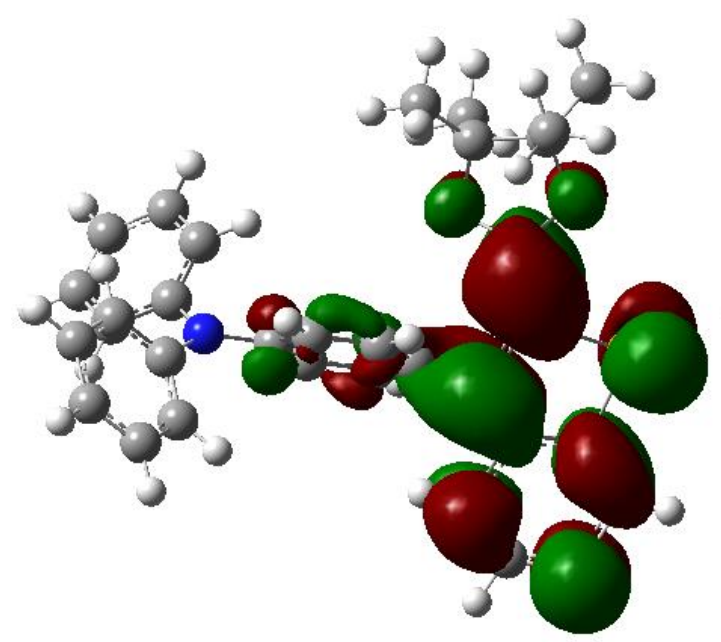

(c) LUMO

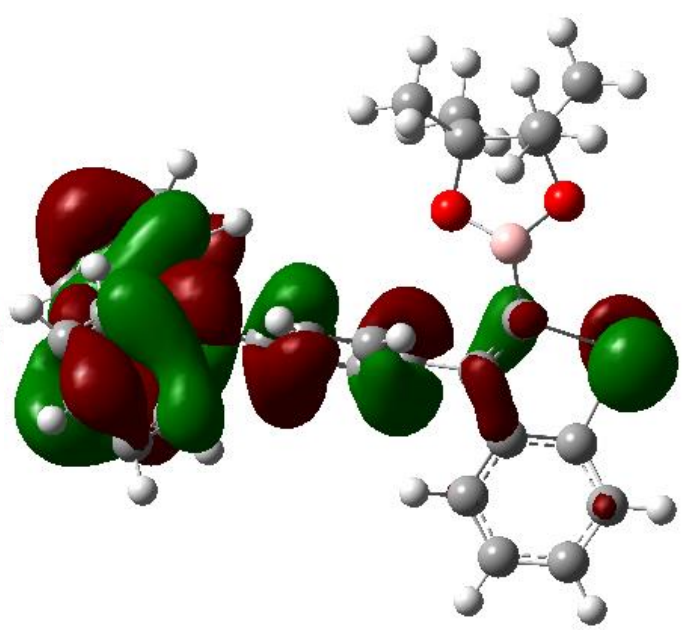

(b) HOMO

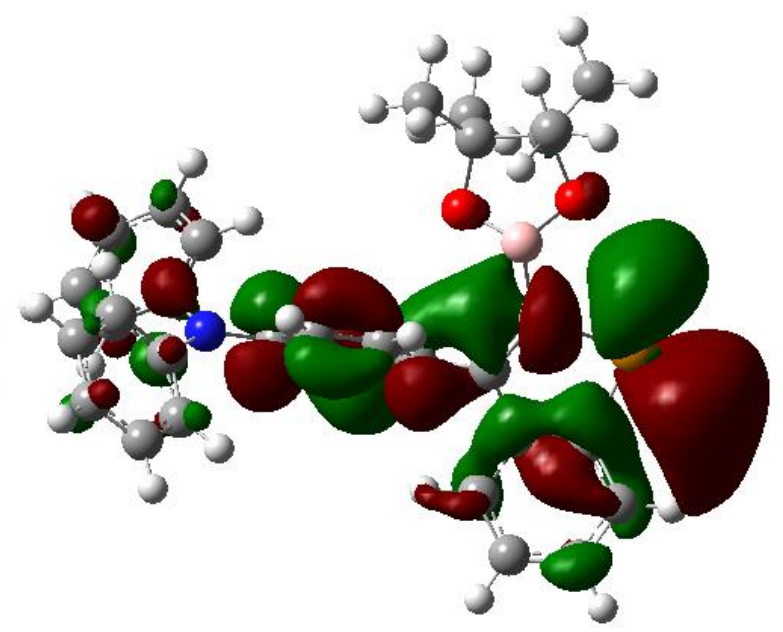

(d) LUMO+1

Figure S35. a) HOMO-1, b) HOMO, c) LUMO, and d) LUMO+1 involved in the main contributions to the lowest two excitations for Benzo-Te(BPin)TPA at the TD-CAM-B3LYP/ccpVTZ-(PP) level of theory at the gas-phase $S_{0}$ geometry (as determined at the B3LYP/cc-pVTZ(PP) level of theory). All orbitals illustrated with an isovalue $=0.02$. 


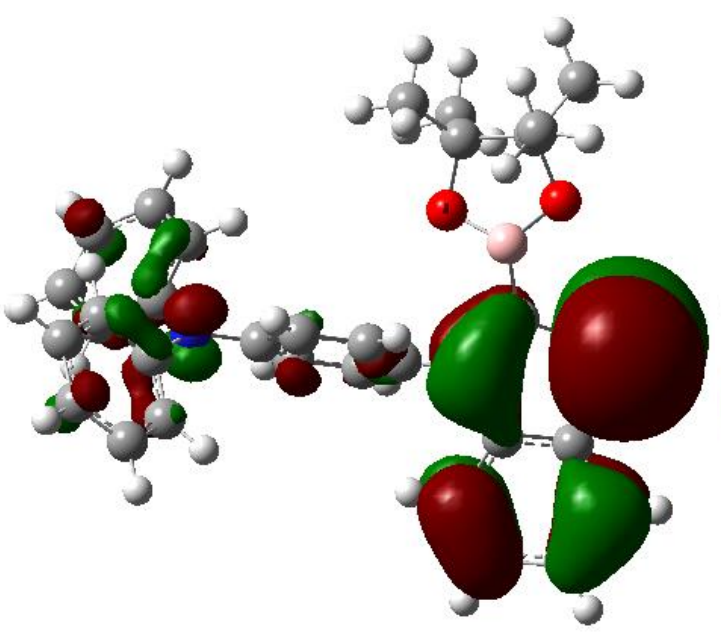

(a) HOMO-1

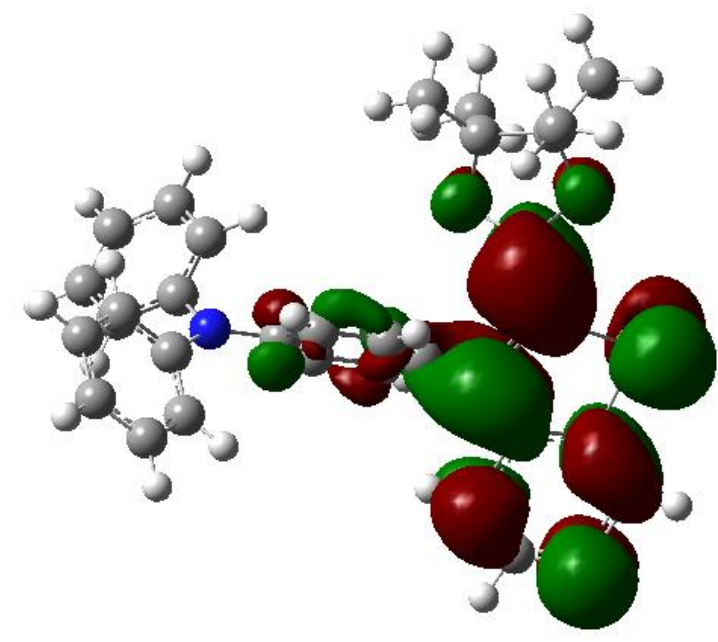

(c) LUMO

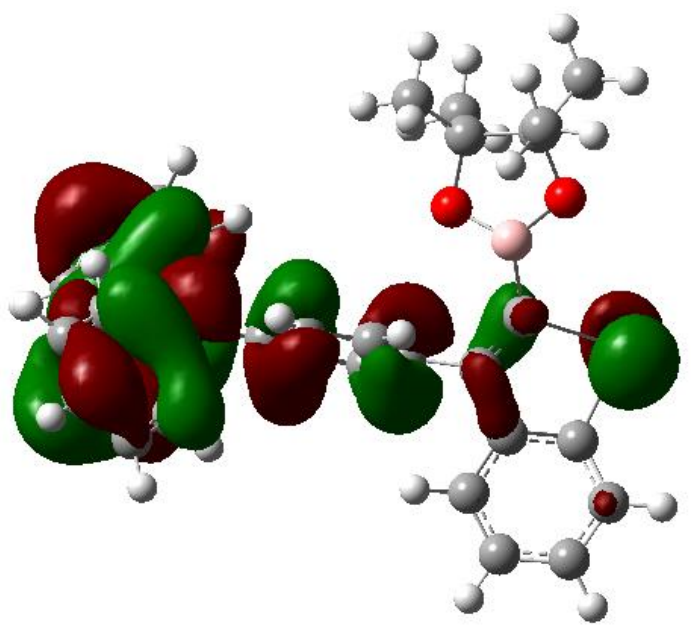

(b) HOMO

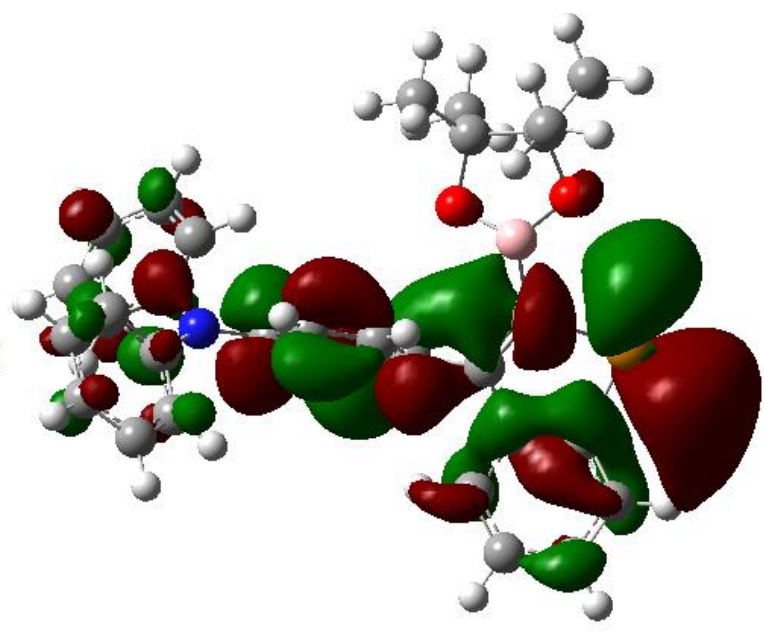

(d) LUMO+1

Figure S36. a) HOMO-1, b) HOMO, c) LUMO, and d) LUMO+1 involved in the main contributions to the lowest two excitations for Benzo-Te(BPin)TPA at the TD-M06-2X/ccpVTZ-(PP) level of theory at the gas-phase $S_{0}$ geometry (as determined at the B3LYP/cc-pVTZ(PP) level of theory). All orbitals illustrated with an isovalue $=0.02$. 
Table S10. Comparison of selected bond lengths $(\AA)$ and angles $\left(^{\circ}\right)$ for $\mathbf{T e C}_{4}(\mathbf{2} \text {-thienyl })_{4}$ determined from the experimental X-ray structure and those computed at the B3LYP/cc-pVTZ(PP) level of theory in the gas-phase. Atom labels are provided in Figure 4 in the main text.

\begin{tabular}{|c|c|c|}
\hline Parameter & Experiment & Theory \\
\hline Te-C(1) & $2.071(5)$ & 2.09147 \\
\hline Te-C(4) & $2.087(5)$ & 2.09455 \\
\hline $\mathrm{C}(2)-\mathrm{C}(3)$ & $1.443(8)$ & 1.45515 \\
\hline $\mathrm{C}(2)-\mathrm{C}(21)$ & $1.493(7)$ & 1.47842 \\
\hline $\mathrm{C}(4)-\mathrm{C}(41)$ & $1.447(8)$ & 1.44759 \\
\hline $\mathrm{C}(1)-\mathrm{Te}-\mathrm{C}(4)$ & $81.9(2)$ & 82.478 \\
\hline $\mathrm{Te}-\mathrm{C}(1)-\mathrm{C}(11) \mathrm{S}(1)$ & 180.0 & 144.65 \\
\hline $\mathrm{Te}-\mathrm{C}(4)-\mathrm{C}(41)-\mathrm{S}(4)$ & 180.0 & -152.70 \\
\hline $\mathrm{Te}-\mathrm{C}(1)-\mathrm{C}(11)$ & $117.3(4)$ & 118.876 \\
\hline $\mathrm{Te}-\mathrm{C}(4)-\mathrm{C}(41)$ & $117.3(4)$ & 118.785 \\
\hline
\end{tabular}

Table S11. The excitation energies ${ }^{\mathrm{a}}$ (in $\mathrm{eV}$ ), absorption wavelengths $(\mathrm{nm})$, and oscillator strengths (f) of the lowest ten lying singlet and triplet states for $\mathbf{T e C}_{\mathbf{4}}(\mathbf{2} \text {-thienyl) })_{4}$ at the TDB3LYP/cc-pVTZ-(PP) level of theory at the gas-phase $\mathrm{S}_{0}$ geometry (as determined at the B3LYP/cc-pVTZ-(PP) level of theory).

\begin{tabular}{cccc} 
Excited state & \multicolumn{4}{c}{$\mathbf{T e C}_{\mathbf{4}}$ (2-thienyl) } \\
\cline { 2 - 4 } & $\mathrm{E} / \mathrm{eV}$ & $\lambda / \mathrm{nm}$ & $\mathrm{f}$ \\
\hline $\mathrm{T}_{1}$ & 1.9740 & 628.09 & 0.0000 \\
$\mathrm{~T}_{2}$ & 2.8429 & 436.12 & 0.0000 \\
$\mathrm{~T}_{3}$ & 3.0294 & 409.26 & 0.0000 \\
$\mathrm{~S}_{1}$ & 3.1314 & 395.93 & 0.4331 \\
$\mathrm{~T}_{4}$ & 3.2317 & 383.65 & 0.0000 \\
$\mathrm{~S}_{2}$ & 3.2375 & 382.96 & 0.0339 \\
$\mathrm{~T}_{5}$ & 3.2872 & 377.17 & 0.0000 \\
$\mathrm{~T}_{6}$ & 3.3757 & 367.29 & 0.0000 \\
$\mathrm{~T}_{7}$ & 3.4103 & 363.55 & 0.0000 \\
$\mathrm{~T}_{8}$ & 3.5630 & 347.98 & 0.0000
\end{tabular}

a) Energies (wavelengths) reported to 4 (2) decimal places to facilitate present and future computational comparison. 


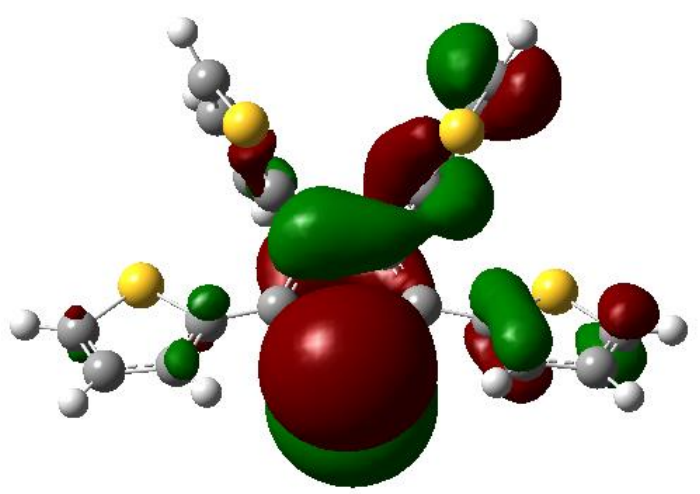

(a) HOMO-1

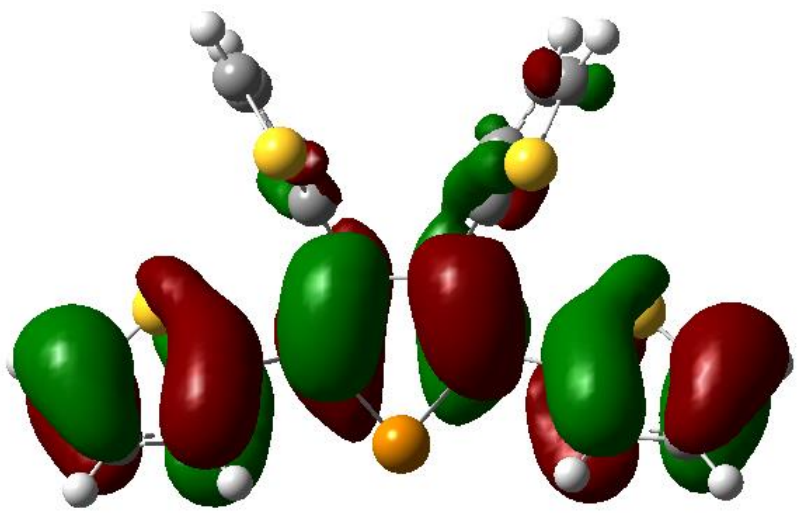

(b) HOMO

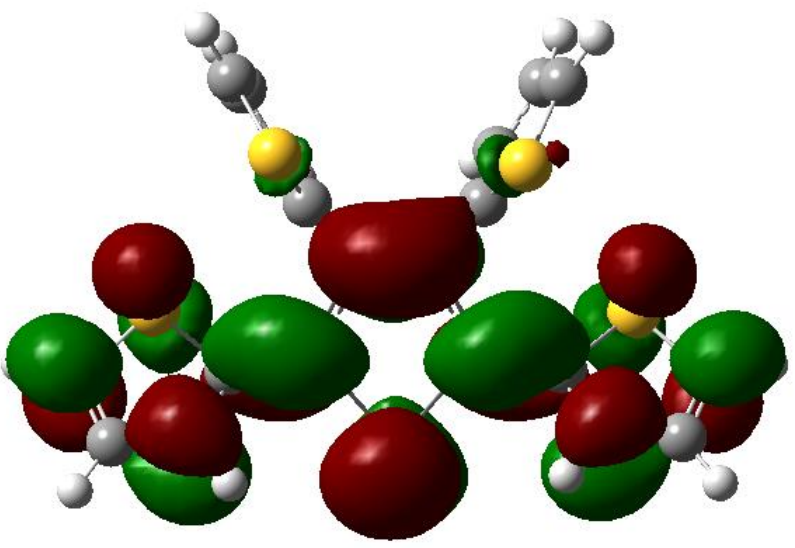

(c) LUMO

Figure S37. a) HOMO-1, b) HOMO, and c) LUMO for $\mathbf{T e C}_{4}$ (2-thienyl) (2) $_{4}$ at the TD-B3LYP/ccpVTZ-(PP) level of theory at the gas-phase $\mathrm{S}_{0}$ geometry (as determined at the B3LYP/cc-pVTZ(PP) level of theory). Excitation to $\mathrm{S}_{1}$ involves HOMO $\rightarrow$ LUMO $(\mathrm{C}=0.68437)$. Excitation to $\mathrm{S}_{3}$ involves HOMO-1 $\rightarrow$ LUMO+1 $(\mathrm{C}=0.65750,3.6113 \mathrm{eV}, 343.32 \mathrm{~nm}, \mathrm{f}=0.0521)$. All orbitals illustrated with an isovalue $=0.02$. 
Table S12. Comparison of selected bond lengths $(\AA)$ and angles $\left({ }^{\circ}\right)$ for Naph-Te-6-Naph determined from the experimental X-ray structure and those computed at the B3LYP/cc-pVDZ(PP) level of theory in the gas-phase. Atom labels are provided in Figure 5 in the main text.

\begin{tabular}{|c|c|c|}
\hline Parameter & Experiment & Theory \\
\hline $\mathrm{C}(1)-\mathrm{C}(2)$ & $1.463(10)$ & 1.44657 \\
\hline $\mathrm{C}(2)-\mathrm{C}(8)$ & $1.349(10)$ & 1.36425 \\
\hline $\mathrm{C}(8)-\mathrm{Te}$ & $2.079(8)$ & 2.09608 \\
\hline $\mathrm{C}(1)-\mathrm{C}(7)$ & $1.359(10)$ & 1.36425 \\
\hline $\mathrm{C}(7)-\mathrm{Te}$ & $2.088(7)$ & 2.09068 \\
\hline $\mathrm{C}(7)-\mathrm{Te}-\mathrm{C}(8)$ & $81.5(3)$ & 82.336 \\
\hline $\mathrm{Te}-\mathrm{C}(7)-\mathrm{C}(12)$ & $118.9(5)$ & 121.083 \\
\hline $\mathrm{Te}-\mathrm{C}(8)-\mathrm{C}(22)$ & $120.7(6)$ & 121.082 \\
\hline $\mathrm{Te}-\mathrm{C}(7)-\mathrm{C}(12)-\mathrm{C}(11)$ & $140.8(6)$ & 127.480 \\
\hline $\mathrm{Te}-\mathrm{C}(8)-\mathrm{C}(22)-\mathrm{C}(23)$ & $119.9(8)$ & 127.489 \\
\hline
\end{tabular}

Table S13. The excitation energies ${ }^{\mathrm{a}}$ (in $\mathrm{eV}$ ), absorption wavelengths $(\mathrm{nm})$, and oscillator strengths (f) of the lowest ten lying singlet and triplet states for Naph-Te-6-Naph at the TDB3LYP/cc-pVTZ-(PP) level of theory at the gas-phase $\mathrm{S}_{0}$ geometry (as determined at the B3LYP/cc-pVDZ-(PP) level of theory).

\begin{tabular}{cccccc}
\multirow{2}{*}{ Excited state } & \multicolumn{3}{l}{ Naph-Te-6-Naph } \\
\cline { 2 - 6 } & $\mathrm{E} / \mathrm{eV}$ & $\lambda / \mathrm{nm}$ & $\mathrm{f}$ & Nature of Excitation \\
\hline $\mathbf{T}_{\mathbf{1}}$ & 2.4241 & 511.47 & 0.0000 & & \\
$\mathbf{T}_{\mathbf{2}}$ & 2.6373 & 470.12 & 0.0000 & & \\
$\mathbf{T}_{\mathbf{3}}$ & 2.9256 & 423.78 & 0.0000 & & \\
$\mathbf{T}_{\mathbf{4}}$ & 3.1828 & 389.55 & 0.0000 & & \\
$\mathbf{T}_{\mathbf{5}}$ & 3.1839 & 389.41 & 0.0000 & & \\
$\mathbf{T}_{\mathbf{6}}$ & 3.3077 & 374.83 & 0.0000 & & \\
$\mathbf{S}_{\mathbf{1}}$ & 3.4087 & 363.73 & 0.0179 & $107 \rightarrow 109$ & 0.66435 \\
& & & & $107 \rightarrow 111$ & 0.16556 \\
$\mathbf{S}_{\mathbf{2}}$ & 3.4887 & 355.38 & 0.3648 & $106 \rightarrow 109$ & 0.34565 \\
& & & & $106 \rightarrow 111$ & 0.12241 \\
& & & & $107 \rightarrow 108$ & 0.59610 \\
$\mathbf{S}_{\mathbf{3}}$ & 3.5557 & 348.69 & 0.2320 & $106 \rightarrow 109$ & 0.55966 \\
& & & & $106 \rightarrow 111$ & 0.20437 \\
$\mathbf{T}_{\mathbf{7}}$ & 3.6676 & 338.05 & 0.0000 & & -0.36328 \\
\end{tabular}

a) Energies (wavelengths) reported to 4 (2) decimal places to facilitate present and future computational comparison.

b) $\mathrm{HOMO}=$ Orbital 107, LUMO = Orbital 108 


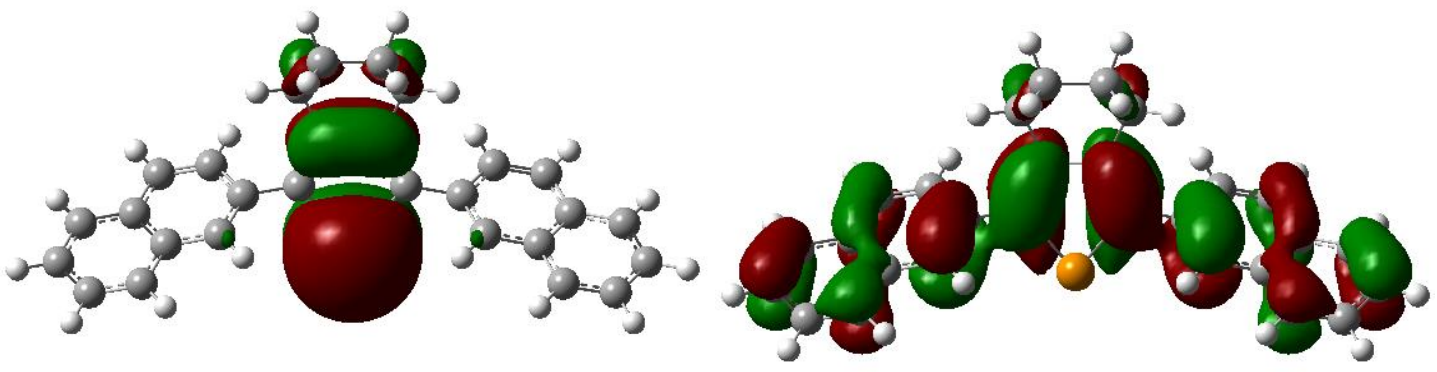

(a) HOMO-1

(b) HOMO
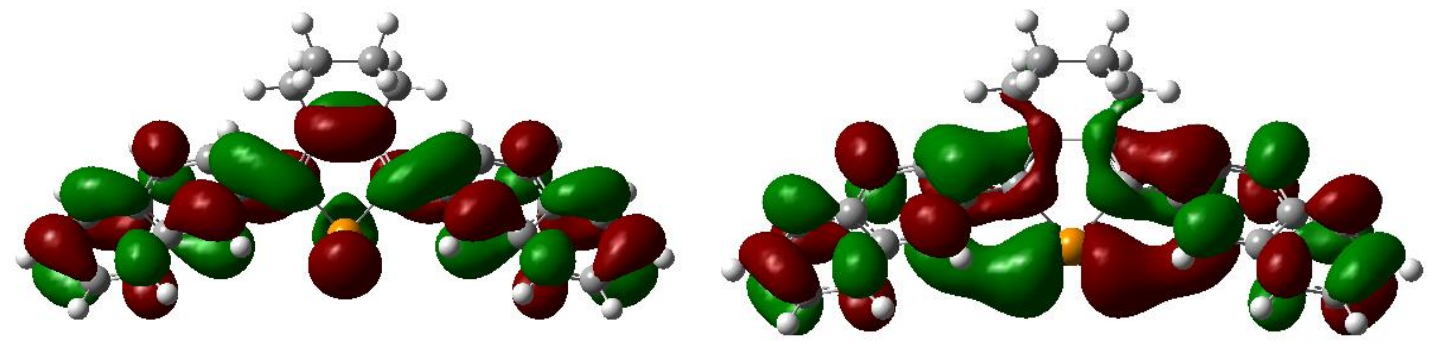

(c) LUMO

(d) LUMO+1

Figure S38. a) HOMO-1, b) HOMO, c) LUMO, and d) $\mathrm{LUMO}+1$, i.e., dominant orbitals involved in the low-lying excited singlet states, for Naph-Te-6-Naph at the TD-B3LYP/ccpVTZ-(PP) level of theory at the gas-phase $\mathrm{S}_{0}$ geometry (as determined at the B3LYP/cc-pVTZ(PP) level of theory). All orbitals illustrated with an isovalue $=0.02$. 
Table S14. The excitation energies ${ }^{\mathrm{a}}$ (in $\mathrm{eV}$ ), absorption wavelengths $(\mathrm{nm})$, and oscillator strengths (f) of the lowest ten lying singlet and triplet states for Fl-Te-6-Fl at the TD-B3LYP/ccpVTZ-(PP) level of theory at the gas-phase $S_{0}$ geometry (as determined at the B3LYP/cc-pVTZ(PP) level of theory).

\begin{tabular}{cccccc} 
Excited state & \multicolumn{5}{c}{ Fl-Te-6-Fl } \\
\cline { 2 - 6 } & $\mathrm{E} / \mathrm{eV}$ & $\lambda / \mathrm{nm}$ & $\mathrm{f}$ & Nature of Excitation \\
\hline $\mathbf{T}_{\mathbf{1}}$ & 2.4541 & 505.22 & 0.0000 & & \\
$\mathbf{T}_{\mathbf{2}}$ & 2.8867 & 429.50 & 0.0000 & & \\
$\mathbf{T}_{\mathbf{3}}$ & 3.1540 & 393.10 & 0.0000 & & \\
$\mathbf{T}_{\mathbf{4}}$ & 3.1904 & 388.62 & 0.0000 & & \\
$\mathbf{T}_{\mathbf{5}}$ & 3.2624 & 380.04 & 0.0000 & & \\
$\mathbf{T}_{\mathbf{6}}$ & 3.3593 & 369.07 & 0.0000 & & \\
$\mathbf{S}_{\mathbf{1}}$ & 3.3894 & 365.80 & 1.3095 & $127 \rightarrow 128$ & 0.69970 \\
$\mathbf{S}_{\mathbf{2}}$ & 3.4936 & 354.89 & 0.0036 & $124 \rightarrow 129$ & 0.10908 \\
& & & & $127 \rightarrow 129$ & 0.63222 \\
& & & & $127 \rightarrow 130$ & 0.25303 \\
& & & & $127 \rightarrow 132$ & 0.11411 \\
$\mathbf{S}_{\mathbf{3}}$ & 3.6603 & 338.72 & 0.0385 & $126 \rightarrow 129$ & 0.62752 \\
& & & & $126 \rightarrow 130$ & 0.26268 \\
& & & & $126 \rightarrow 132$ & 0.15472 \\
$\mathbf{S}_{\mathbf{4}}$ & 3.7037 & 334.76 & 0.0237 & $126 \rightarrow 128$ & 0.69393
\end{tabular}
a) Energies (wavelengths) reported to 4 (2) decimal places to facilitate present and future computational comparison.
b) $\mathrm{HOMO}=$ Orbital 127, LUMO $=$ Orbital 128

Table S15. Comparison of selected bond lengths $(\AA)$ and angles $\left(^{\circ}\right)$ for $\mathbf{A r} \mathbf{r}^{\mathbf{F}}-\mathbf{T e}-\mathbf{6}-\mathbf{A r} \mathbf{r}^{\mathbf{F}}$ determined from the experimental X-ray structure and those computed at the B3LYP/cc-pVDZ(PP) level of theory in the gas-phase. Values due to a second molecule in the asymmetric unit in square brackets. Atom labels are provided in Figure 6 in the main text.

\begin{tabular}{|c|c|c|}
\hline Parameter & Experiment & Theory \\
\hline Te-C(7) & $2.068(3)[2.069(3)]$ & 2.09486 \\
\hline $\mathrm{Te}-\mathrm{C}(8)$ & $2.068(3)[2.075(3)]$ & 2.09486 \\
\hline $\mathrm{C}(1)-\mathrm{C}(2)$ & $1.449(4)[1.452(4)]$ & 1.44603 \\
\hline $\mathrm{C}(1)-\mathrm{C}(7)$ & $1.366(4)[1.360(4)]$ & 1.36457 \\
\hline $\mathrm{C}(2)-\mathrm{C}(8)$ & $1.366(4)[1.361(4)]$ & 1.36457 \\
\hline $\mathrm{C}(7)-\mathrm{Te}-\mathrm{C}(8)$ & $81.66(12)[81.48(12)]$ & 81.976 \\
\hline $\mathrm{Te}-\mathrm{C}(7)-\mathrm{C}(11)$ & $122.3(2)[121.9(2)]$ & 121.067 \\
\hline $\mathrm{Te}-\mathrm{C}(8)-\mathrm{C}(21)$ & $124.8(3)[121.3(2)]$ & 121.068 \\
\hline $\mathrm{C}(1)-\mathrm{C}(7)-\mathrm{C}(11)-\mathrm{C}(12)$ & $115.8(4)[119.6(4)]$ & 130.695 \\
\hline $\mathrm{C}(2)-\mathrm{C}(8)-\mathrm{C}(21)-\mathrm{C}(22)$ & $114.5(3)[127.1(3)]$ & 130.685 \\
\hline
\end{tabular}


Table S16. The excitation energies ${ }^{\mathrm{a}}$ (in $\mathrm{eV}$ ), absorption wavelengths $(\mathrm{nm})$, and oscillator strengths (f) of the lowest ten lying singlet and triplet states for $\mathbf{A r} \mathbf{r}^{\mathbf{F}}-\mathbf{T e}-\mathbf{6}-\mathbf{A r} \mathbf{r}^{\mathbf{F}}$ at the TDB3LYP/cc-pVTZ-(PP) level of theory at the gas-phase $\mathrm{S}_{0}$ geometry (as determined at the B3LYP/cc-pVDZ-(PP) level of theory).

\begin{tabular}{cccccc}
\multirow{2}{*}{ Excited state } & \multicolumn{6}{c}{ Ar $\mathbf{F}-\mathbf{T e}-\mathbf{6}-\mathbf{A r} \mathbf{r}^{\mathbf{F}}$} & & \\
\cline { 2 - 6 } & $\mathrm{E} / \mathrm{eV}$ & $\lambda / \mathrm{nm}$ & $\mathrm{f}$ & Nature of Excitation $^{\mathrm{b}}$ \\
\hline $\mathbf{T}_{\mathbf{1}}$ & 2.6314 & 471.17 & 0.0000 & & \\
$\mathbf{T}_{\mathbf{2}}$ & 3.1082 & 398.90 & 0.0000 & & \\
$\mathbf{T}_{\mathbf{3}}$ & 3.1585 & 392.54 & 0.0000 & & \\
$\mathbf{T}_{\mathbf{4}}$ & 3.3030 & 375.37 & 0.0000 & & \\
$\mathbf{S}_{\mathbf{1}}$ & 3.5317 & 351.06 & 0.0000 & $145 \rightarrow 147$ & 0.67493 \\
& & & & $145 \rightarrow 149$ & 0.12666 \\
& & & & $145 \rightarrow 150$ & 0.15111 \\
$\mathbf{T}_{\mathbf{5}}$ & 3.6146 & 343.01 & 0.0000 & & \\
$\mathbf{S}_{\mathbf{2}}$ & 3.6485 & 339.82 & 0.0021 & $144 \rightarrow 147$ & 0.66153 \\
& & & & $144 \rightarrow 150$ & 0.11338 \\
& & & & $145 \rightarrow 146$ & -0.18316 \\
$\mathbf{T}_{\mathbf{6}}$ & 3.6696 & 337.87 & 0.0000 & & \\
$\mathbf{S}_{\mathbf{3}}$ & 3.6974 & 335.32 & 0.0387 & $144 \rightarrow 147$ & 0.17389 \\
& & & & $145 \rightarrow 146$ & 0.67315 \\
$\mathbf{T}_{\mathbf{8}}$ & 3.7632 & 329.47 & 0.0000 & & \\
$\mathbf{S}_{\mathbf{4}}$ & 3.7995 & 326.32 & 0.5305 & $144 \rightarrow 146$ & 0.69285
\end{tabular}
a) Energies (wavelengths) reported to 4 (2) decimal places to facilitate present and future computational comparison.
b) $\mathrm{HOMO}=$ Orbital 145 and LUMO = Orbital 146 


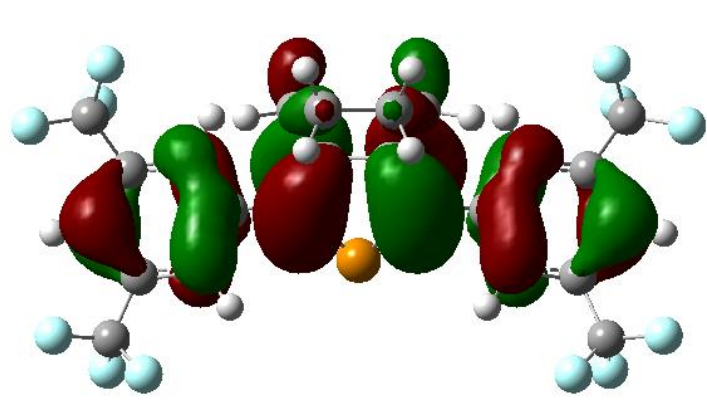

HOMO-1

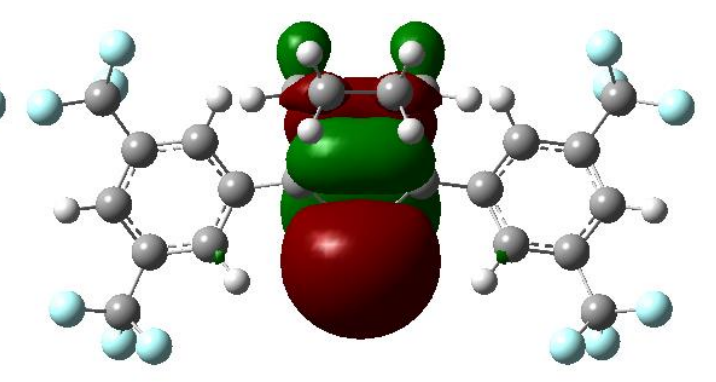

HOMO

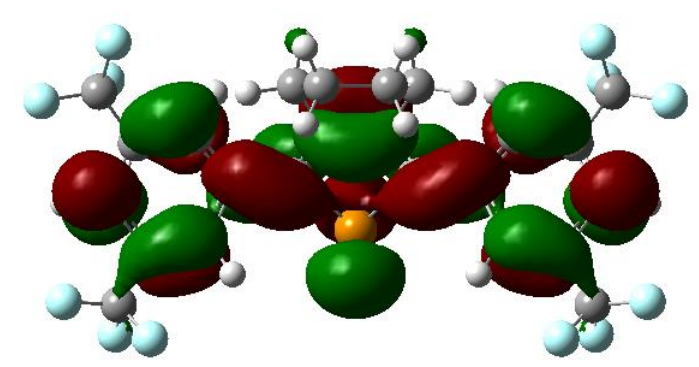

LUMO

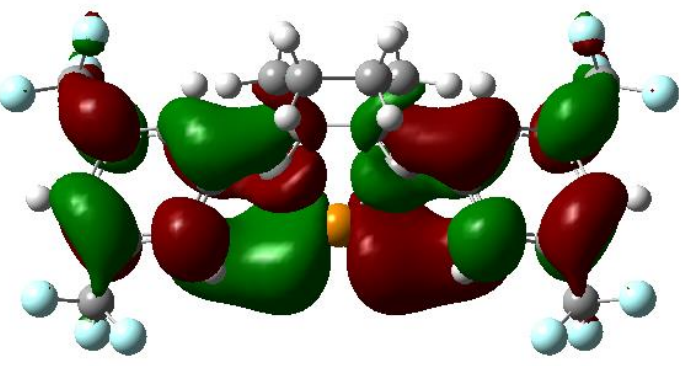

LUMO+1

Figure S39. HOMO-1, HOMO, LUMO, and LUMO+1, i.e., dominant orbitals involved in the low-lying excited singlet states, for $\mathbf{A} \mathbf{r}^{\mathbf{F}}-\mathbf{T e}-\mathbf{6}-\mathbf{A r} \mathbf{r}^{\mathbf{F}}$ at the TD-B3LYP/cc-pVTZ-(PP) level of theory at the gas-phase $\mathrm{S}_{0}$ geometry (as determined at the B3LYP/cc-pVTZ-(PP) level of theory). All orbitals illustrated with an isovalue $=0.02$.

Table S17. The phosphorescence energies (in eV) and corresponding wavelengths (in brackets in $\mathrm{nm}$ ) for select compounds as determined at the (U)B3LYP/cc-pVTZ-(PP) level of theory. Phosphorescence energies determined from (i) the singlet to triplet energy gap at the optimized geometry for the triplet state $\left(\mathrm{E}_{\mathrm{vertical}}\right)$; (ii) the energy difference between the lowest energy triplet and ground state singlet at their corresponding optimized geometries ( $\mathrm{E}_{\text {adiabatic }}$ ); or (iii) the energy difference between the lowest energy triplet and ground state singlet at their corresponding optimized geometries including the zero-point vibrational energy difference $\left(\mathrm{E}_{0-0}\right)$.

\begin{tabular}{|l|c|c|c|c|c|}
\hline & $\begin{array}{c}\text { TPA-6-Te- } \\
\text { TPA }\end{array}$ & $\begin{array}{c}\text { Benzo- } \\
\text { Te(BPin)TPA }\end{array}$ & Naph-Te-6-Naph & Ar $^{\mathrm{F}}$-Te-6-Ar & Fl-Te-6-Fl \\
\hline $\mathrm{E}_{\text {vertical }}$ & $1.28(970)$ & $1.84(674)$ & $1.30(953)$ & $1.33(930)$ & $1.29(961)$ \\
\hline $\mathrm{E}_{\text {adiabatic }}$ & $1.87(628)$ & $2.30(539)$ & $2.02(615)$ & $2.12(586)$ & $2.00(619)$ \\
\hline $\mathrm{E}_{0-0}$ & $1.90(654)$ & $2.24(554)$ & $1.94(640)$ & $2.03(610)$ & $1.92(646)$ \\
\hline Experimental & $2.38(520)$ & $2.08(595)$ & $2.11(588) / 1.96(634)^{\mathrm{a}}$ & $2.08(595)$ & $1.96(633)$ \\
\hline
\end{tabular}

a) Emission in the solid-state exhibits two peaks. 
Table S18. The excitation energies ${ }^{\mathrm{a}}$ (in $\mathrm{eV}$ ), absorption wavelengths $(\mathrm{nm})$, and oscillator strengths (f) of the lowest ten lying singlet and triplet states for $4 \mathrm{BTe}$, 4BSe, and $4 \mathrm{BS}$ at the TDB3LYP/cc-pVTZ-(PP) level of theory at the gas-phase $\mathrm{S}_{0}$ geometry (as determined at the B3LYP/cc-pVTZ-(PP) level of theory).

\begin{tabular}{|c|c|c|c|c|c|c|c|c|}
\hline & $\begin{array}{c}\text { 4BTe } \\
\text { energy }(e V)\end{array}$ & f & & $\begin{array}{c}\text { 4BSe } \\
\text { energy }(e V\end{array}$ & f & & $\begin{array}{c}\text { 4BS } \\
\text { energy }(e V)\end{array}$ & f \\
\hline & \multicolumn{8}{|c|}{ Gas-phase } \\
\hline T1 & 2.7398 & 0 & T1 & 2.927 & 0 & T1 & 3.0886 & 0 \\
\hline $\mathbf{T} 2$ & 2.8095 & 0 & T2 & 3.2917 & 0 & $\mathbf{T} 2$ & 3.6704 & 0 \\
\hline S1 & 3.7396 & 0.0714 & S1 & 4.3744 & 0.0721 & S1 & 4.7443 & 0.2642 \\
\hline T3 & 3.8663 & 0 & S2 & 4.5566 & 0.241 & T3 & 4.7645 & 0 \\
\hline $\mathbf{S 2}$ & 4.2565 & 0.1927 & T3 & 4.7292 & 0 & S2 & 4.78 & 0.0726 \\
\hline \multirow[t]{2}{*}{ S3 } & 4.3651 & 0.0068 & T4 & 4.8835 & 0 & T4 & 4.9971 & 0 \\
\hline & \multicolumn{8}{|c|}{$\mathrm{CHCl}_{3}$} \\
\hline T1 & 2.7788 & 0 & T1 & 2.9409 & 0 & T1 & 3.097 & 0 \\
\hline $\mathbf{T} 2$ & 2.845 & 0 & $\mathbf{T 2}$ & 3.3338 & 0 & $\mathbf{T} 2$ & 3.7062 & 0 \\
\hline S1 & 3.7499 & 0.1149 & S1 & 4.3658 & 0.1077 & S1 & 4.7088 & 0.3556 \\
\hline T3 & 3.8884 & 0 & S2 & 4.5313 & 0.3076 & $\mathbf{S 2}$ & 4.7825 & 0.0845 \\
\hline $\mathbf{S 2}$ & 4.3092 & 0.2195 & T3 & 4.7819 & 0 & T3 & 4.8214 & 0 \\
\hline \multirow[t]{2}{*}{ S3 } & 4.3518 & 0.0085 & T4 & 4.969 & 0 & T4 & 5.0812 & 0 \\
\hline & \multicolumn{8}{|c|}{ THF } \\
\hline T1 & 2.7824 & 0 & T1 & 2.9436 & 0 & T1 & 3.0983 & 0 \\
\hline T2 & 2.8513 & 0 & T2 & 3.3405 & 0 & $\mathbf{T} 2$ & 3.7122 & 0 \\
\hline S1 & 3.7603 & 0.1116 & S1 & 4.3755 & 0.1054 & S1 & 4.715 & 0.3495 \\
\hline T3 & 3.8919 & 0 & S2 & 4.5387 & 0.3036 & S2 & 4.7919 & 0.0849 \\
\hline S2 & 4.3167 & 0.2164 & T3 & 4.7913 & 0 & T3 & 4.8318 & 0 \\
\hline S3 & 4.3566 & 0.0082 & T4 & 4.9855 & 0 & T4 & 5.098 & 0 \\
\hline
\end{tabular}

a) Energies (wavelengths) reported to 4 (2) decimal places to facilitate present and future computational comparison. 
Table S19. Vertical excitation energies (in eV), oscillator strengths (f), and dominant transitions for the two lowest lying singlet states for 4BS, 4BSe, and 4BTe, as computed at the TDB3LYP/aug-cc-pVTZ-(PP) level of theory in THF (IEF-PCM).

\begin{tabular}{|l|l|l|l|l|}
\hline Compound & State & Vertical Excitation (eV) & Oscillator Strength (f) & Excitation $^{\text {a }}$ \\
\hline \multirow{3}{*}{ S-B4 } & $\mathrm{S}_{1}$ & 4.7150 & 0.3495 & $\mathrm{H} \rightarrow \mathrm{L}$ \\
\cline { 2 - 5 } & $\mathrm{S}_{2}$ & 4.7919 & 0.0849 & $\mathrm{H}-1 \rightarrow \mathrm{L}$ \\
\hline \multirow{3}{*}{ Se-B4 } & $\mathrm{S}_{1}$ & 4.3755 & 0.1054 & $\mathrm{H} \rightarrow \mathrm{L}$ \\
\cline { 2 - 5 } & $\mathrm{S}_{2}$ & 4.5387 & 0.3036 & $\mathrm{H}-1 \rightarrow \mathrm{L}$ \\
\hline \multirow{2}{*}{ Te-B4 } & $\mathrm{S}_{1}$ & 3.7603 & 0.1116 & $\mathrm{H} \rightarrow \mathrm{L}$ \\
\cline { 2 - 5 } & $\mathrm{S}_{2}$ & 4.3167 & 0.2164 & $\mathrm{H}-1 \rightarrow \mathrm{L}$ \\
\hline
\end{tabular}

a. $\mathrm{H}=\mathrm{HOMO} ; \mathrm{L}=\mathrm{LUMO}$

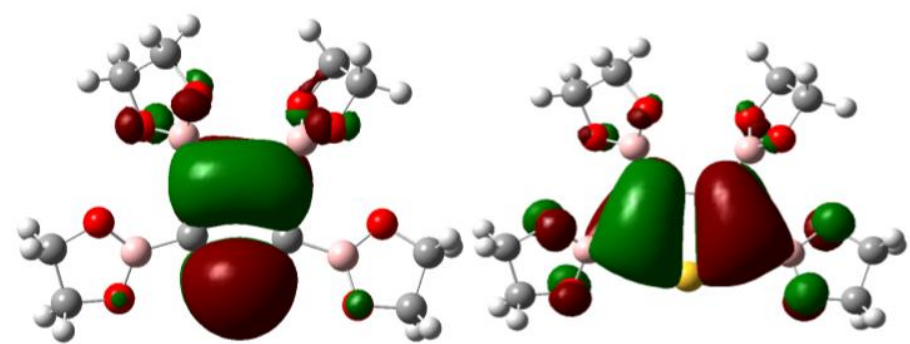

HOMO-1

HOMO

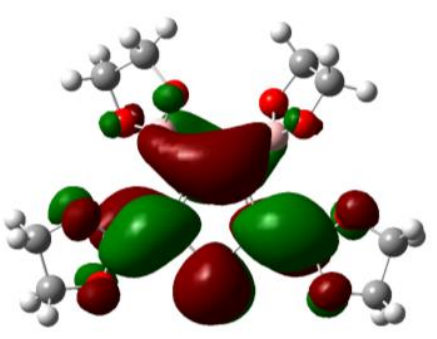

LUMO

Figure S40. Molecular orbitals, as computed at the TD-B3LYP/aug-cc-pVTZ-(PP) level of theory in THF (IEF-PCM), involved in the main excitations for 4BS. All orbitals illustrated with an isovalue $=0.02$.

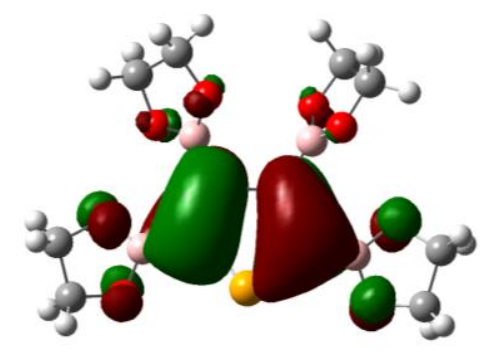

HOMO-1

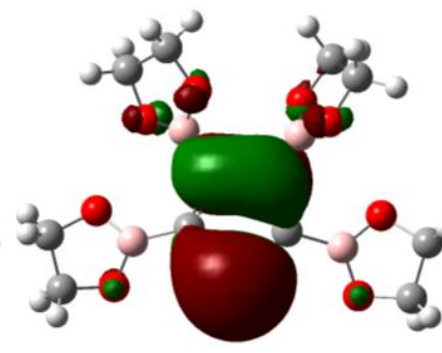

HOMO

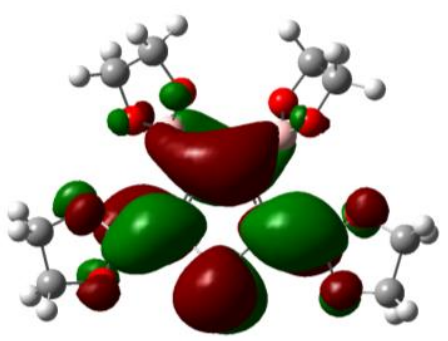

LUMO

Figure S41. Molecular orbitals, as computed at the TD-B3LYP/aug-cc-pVTZ-(PP) level of theory in THF (IEF-PCM), involved in the main excitations for 4BSe. All orbitals illustrated with an isovalue $=0.02$. 


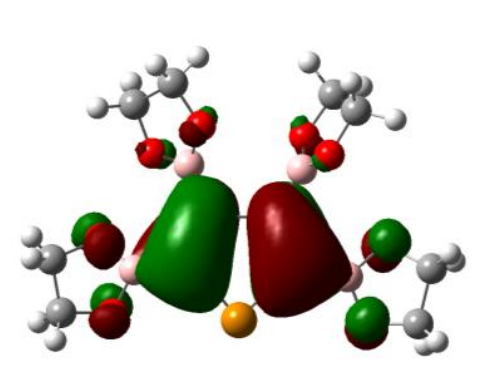

HOMO-1

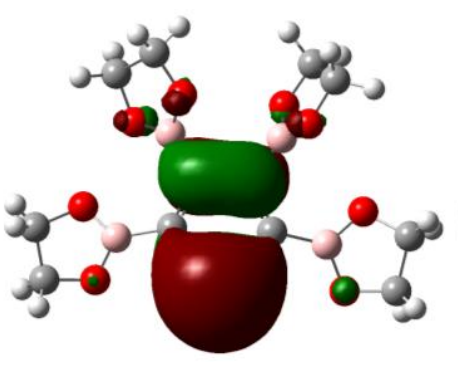

HOMO

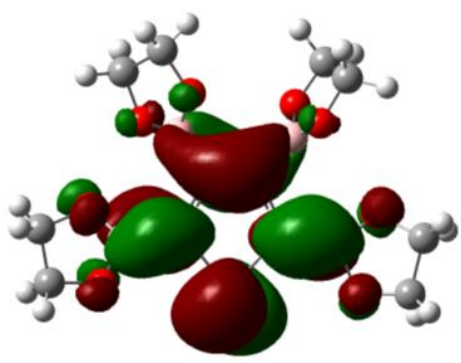

LUMO

Figure S42. Molecular orbitals, as computed at the TD-B3LP/aug-cc-pVTZ-(PP) level of theory in THF (IEF-PCM), involved in the main excitations for 4BTe. All orbitals illustrated with an isovalue $=0.02$.

Table S20. Gas phase B3LYP/cc-pVTZ (cc-pVTZ-PP Te) determined xyz coordinates (in $\AA$ ) for TPA-Te-6-TPA at $\mathrm{S}_{0}$ equilibrium geometry.

$\begin{array}{lrrr}\mathrm{Te} & 0.00951300 & -0.90538900 & 0.08548900 \\ \mathrm{C} & -0.73828700 & 1.86166500 & 0.05967800 \\ \mathrm{C} & 0.70867200 & 1.87455900 & 0.05816000 \\ \mathrm{C} & 0.73987100 & 4.08376900 & 1.25437400 \\ \mathrm{H} & 1.11350100 & 5.10583200 & 1.17230300 \\ \mathrm{H} & 1.10591700 & 3.69864400 & 2.20718500 \\ \mathrm{C} & -0.81012500 & 4.07253000 & 1.25200900 \\ \mathrm{H} & -1.17342400 & 3.68644800 & 2.20546400 \\ \mathrm{H} & -1.19827400 & 5.08870400 & 1.16409900 \\ \mathrm{C} & -1.41029400 & 3.21153900 & 0.12386900 \\ \mathrm{H} & -1.27578000 & 3.72908500 & -0.83257300 \\ \mathrm{H} & -2.48343100 & 3.10795900 & 0.26965000 \\ \mathrm{C} & 1.35631600 & 3.23625500 & 0.12482800 \\ \mathrm{H} & 2.43089100 & 3.15183600 & 0.27235900 \\ \mathrm{H} & 1.21383100 & 3.75335600 & -0.83079200 \\ \mathrm{C} & 4.79716200 & -0.71906400 & 0.86241400 \\ \mathrm{C} & 2.82370700 & 0.43880200 & 0.03356300 \\ \mathrm{C} & 5.61187700 & -0.07055600 & -0.07162400 \\ \mathrm{H} & 5.23562100 & -1.42518600 & 1.55311500 \\ \mathrm{C} & 3.64837300 & 1.06982500 & -0.90888800 \\ \mathrm{C} & 5.01203800 & 0.83182900 & -0.95568300 \\ \mathrm{~N} & 7.00310100 & -0.32682600 & -0.12608900 \\ \mathrm{H} & 3.20932400 & 1.75130300 & -1.62406800 \\ \mathrm{H} & 5.61864100 & 1.33989400 & -1.69185900 \\ \mathrm{C} & 7.74465200 & -0.48014000 & 1.07347200 \\ \mathrm{C} & 7.65651400 & -0.44143300 & -1.38014100\end{array}$




\begin{tabular}{|c|c|c|c|}
\hline $\mathrm{C}$ & 8.70637800 & -1.48838600 & 1.19219300 \\
\hline $\mathrm{C}$ & 7.52649500 & 0.37409000 & 2.15909600 \\
\hline $\mathrm{C}$ & 8.90773600 & 0.14821800 & -1.58646900 \\
\hline $\mathrm{C}$ & 7.06174400 & -1.14543100 & -2.43196000 \\
\hline $\mathrm{C}$ & 9.43367700 & -1.63071900 & 2.36559800 \\
\hline $\mathrm{H}$ & 8.87993200 & -2.15797200 & 0.36174600 \\
\hline $\mathrm{C}$ & 8.24687200 & 0.21464900 & 3.33445600 \\
\hline $\mathrm{H}$ & 6.79051500 & 1.16140100 & 2.07697300 \\
\hline $\mathrm{C}$ & 9.54722100 & 0.02800800 & -2.81228200 \\
\hline $\mathrm{H}$ & 9.37486000 & 0.69948300 & -0.78270300 \\
\hline $\mathrm{C}$ & 7.70112400 & -1.24753600 & -3.65939400 \\
\hline $\mathrm{H}$ & 6.09764000 & -1.61058000 & -2.28301400 \\
\hline $\mathrm{C}$ & 9.20732600 & -0.78474400 & 3.44573900 \\
\hline $\mathrm{H}$ & 10.17238200 & -2.41802700 & 2.43902600 \\
\hline $\mathrm{H}$ & 8.06436800 & 0.88598200 & 4.16326200 \\
\hline $\mathrm{C}$ & 8.94844900 & -0.66581700 & -3.85804800 \\
\hline $\mathrm{H}$ & 10.51451600 & 0.49216200 & -2.95322400 \\
\hline $\mathrm{H}$ & 7.22518500 & -1.79726900 & -4.46074100 \\
\hline $\mathrm{H}$ & 9.77128600 & -0.90253200 & 4.36078100 \\
\hline $\mathrm{H}$ & 9.44673800 & -0.75201800 & -4.81383800 \\
\hline $\mathrm{C}$ & -5.02256800 & 0.72441300 & -0.94722000 \\
\hline $\mathrm{C}$ & -2.82731400 & 0.38893200 & 0.04739700 \\
\hline $\mathrm{C}$ & -5.60704000 & -0.16393200 & -0.03895300 \\
\hline $\mathrm{H}$ & -5.63649800 & 1.19519400 & -1.70198400 \\
\hline $\mathrm{C}$ & -3.42304300 & -0.51133600 & 0.94169200 \\
\hline $\mathrm{C}$ & -4.78147900 & -0.77714200 & 0.90913400 \\
\hline $\mathrm{N}$ & -6.99443600 & -0.44052000 & -0.08301200 \\
\hline $\mathrm{H}$ & -2.81110400 & -0.98899200 & 1.69589600 \\
\hline $\mathrm{H}$ & -5.21099400 & -1.46305300 & 1.62545300 \\
\hline $\mathrm{C}$ & -7.92089900 & 0.60456500 & -0.33156100 \\
\hline $\mathrm{C}$ & -7.46193800 & -1.76553400 & 0.11456000 \\
\hline $\mathrm{C}$ & -9.00165800 & 0.40584700 & -1.19653500 \\
\hline $\mathrm{C}$ & -7.77108200 & 1.85112700 & 0.28433000 \\
\hline $\mathrm{C}$ & -8.58676300 & -2.01158300 & 0.90797300 \\
\hline $\mathrm{C}$ & -6.80795300 & -2.84804400 & -0.48202000 \\
\hline $\mathrm{C}$ & -9.91060800 & 1.42799400 & -1.43098800 \\
\hline $\mathrm{H}$ & -9.12539500 & -0.55177600 & -1.68215200 \\
\hline $\mathrm{C}$ & -8.67632200 & 2.87256800 & 0.03270200 \\
\hline $\mathrm{H}$ & -6.94333200 & 2.01385200 & 0.95997100 \\
\hline $\mathrm{C}$ & -9.04647100 & -3.30807200 & 1.09196600 \\
\hline $\mathrm{H}$ & -9.09778100 & -1.18318100 & 1.37787500 \\
\hline $\mathrm{C}$ & -7.26537500 & -4.14271900 & -0.28062100 \\
\hline $\mathrm{H}$ & -5.94133700 & -2.66929000 & -1.10269400 \\
\hline $\mathrm{C}$ & -9.75350800 & 2.66845800 & -0.82259100 \\
\hline $\mathrm{H}$ & -10.74003700 & 1.25571600 & -2.10426600 \\
\hline $\mathrm{H}$ & -8.54460700 & 3.82973500 & 0.51997900 \\
\hline $\mathrm{C}$ & -8.38851600 & -4.38244100 & 0.50346600 \\
\hline $\mathrm{H}$ & -9.91775400 & -3.47873600 & 1.71056100 \\
\hline $\mathrm{H}$ & -6.74676900 & -4.96734500 & -0.75175200 \\
\hline $\mathrm{H}$ & -10.46054400 & 3.46436900 & -1.01203000 \\
\hline $\mathrm{H}$ & -8.74590400 & -5.39190400 & 0.65344900 \\
\hline
\end{tabular}




$\begin{array}{lrrr}\mathrm{C} & 1.37861700 & 0.68591900 & 0.07066700 \\ \mathrm{C} & -1.38665400 & 0.66126300 & 0.07666300 \\ \mathrm{C} & -3.66523800 & 0.99540700 & -0.89947300 \\ \mathrm{H} & -3.23652200 & 1.66259700 & -1.63429400 \\ \mathrm{C} & 3.43749200 & -0.46297100 & 0.91391000 \\ \mathrm{H} & 2.83394100 & -0.97180600 & 1.65437300\end{array}$

Table S21. Gas phase B3LYP/cc-pVTZ (cc-pVTZ-PP Te) determined xyz coordinates (in $\AA$ ) for Benzo-Te(BPin)TPA at $S_{0}$ equilibrium geometry.

\begin{tabular}{cccc}
$\mathrm{Te}$ & 4.54777200 & -0.71353400 & -0.03727000 \\
$\mathrm{O}$ & 1.11091000 & 2.10291100 & 0.26051400 \\
$\mathrm{O}$ & 3.38094200 & 2.29282500 & 0.44109600 \\
$\mathrm{C}$ & 3.62594600 & -2.55117300 & -0.41001600 \\
$\mathrm{C}$ & 2.21868200 & -2.42596000 & -0.40028200 \\
$\mathrm{C}$ & 2.54448000 & -0.06926300 & 0.04953500 \\
$\mathrm{C}$ & 1.38792100 & 3.53276700 & 0.23414800 \\
$\mathrm{C}$ & 2.85647600 & 3.60121200 & 0.80154800 \\
$\mathrm{~B}$ & 2.31263300 & 1.44178200 & 0.25582000 \\
$\mathrm{C}$ & 4.23919400 & -3.78262500 & -0.62971600 \\
$\mathrm{C}$ & 3.45691700 & -4.90661500 & -0.83882900 \\
$\mathrm{C}$ & 2.06262300 & -4.80658000 & -0.82047700 \\
$\mathrm{C}$ & 1.45097200 & -3.58705000 & -0.60163000 \\
$\mathrm{H}$ & 5.31776400 & -3.86778000 & -0.63619200 \\
$\mathrm{H}$ & 3.92876700 & -5.86496200 & -1.01133900 \\
$\mathrm{H}$ & 1.45810900 & -5.69023100 & -0.97496700 \\
$\mathrm{H}$ & 0.37252600 & -3.52010800 & -0.58114000 \\
$\mathrm{C}$ & 2.91632100 & 3.69402000 & 2.32711300 \\
$\mathrm{H}$ & 3.94436400 & 3.53141000 & 2.64877200 \\
$\mathrm{H}$ & 2.59691400 & 4.67363600 & 2.68232300 \\
$\mathrm{H}$ & 2.29212400 & 2.93467000 & 2.79663400 \\
$\mathrm{C}$ & 3.74365700 & 4.66925800 & 0.17769400 \\
$\mathrm{H}$ & 3.34038700 & 5.66543300 & 0.36588800 \\
$\mathrm{H}$ & 4.73862000 & 4.61994600 & 0.61939900 \\
$\mathrm{H}$ & 3.84515400 & 4.53208100 & -0.89596200 \\
$\mathrm{C}$ & 0.33416000 & 4.24281800 & 1.07232100 \\
$\mathrm{H}$ & 0.53655500 & 5.31372300 & 1.12449800 \\
$\mathrm{H}$ & -0.64510000 & 4.10706500 & 0.61427300 \\
$\mathrm{H}$ & 0.28878800 & 3.84739300 & 2.08394600 \\
$\mathrm{C}$ & 1.28091700 & 3.97329300 & -1.22691600 \\
$\mathrm{H}$ & 0.29171500 & 3.71152600 & -1.60009400 \\
$\mathrm{H}$ & 1.41409500 & 5.04998400 & -1.33252300 \\
$\mathrm{H}$ & 2.01932800 & 3.46980000 & -1.84974900 \\
$\mathrm{C}$ & 0.19533900 & -0.89507700 & -0.12986400 \\
$\mathrm{C}$ & -0.57513500 & -1.04217500 & -1.28613900 \\
$\mathrm{C}$ & -0.46553600 & -0.56176800 & 1.05279600 \\
$\mathrm{C}$ & -1.94925700 & -0.86242900 & -1.26412400 \\
$\mathrm{H}$ & -0.09226900 & -1.30256500 & -2.21888400 \\
$\mathrm{C}$ & -1.83802200 & -0.37090600 & 1.08337000 \\
& & & \\
\hline
\end{tabular}




$\begin{array}{cccc}\mathrm{H} & 0.10692000 & -0.43709600 & 1.96181400 \\ \mathrm{C} & -2.60335100 & -0.51996200 & -0.07637900 \\ \mathrm{H} & -2.52139000 & -0.98501700 & -2.17279800 \\ \mathrm{H} & -2.32215100 & -0.10338600 & 2.01183100 \\ \mathrm{C} & 1.67089700 & -1.09703000 & -0.15763600 \\ \mathrm{C} & -4.77240400 & -0.79857200 & 1.04649800 \\ \mathrm{C} & -5.79613700 & -0.01324700 & 1.58611900 \\ \mathrm{C} & -4.52011800 & -2.05410000 & 1.60861300 \\ \mathrm{C} & -6.55039200 & -0.47774600 & 2.65443900 \\ \mathrm{H} & -5.99769600 & 0.96064300 & 1.16304700 \\ \mathrm{C} & -5.26889700 & -2.50448200 & 2.68679000 \\ \mathrm{H} & -3.73549500 & -2.67288400 & 1.19691000 \\ \mathrm{C} & -6.29075900 & -1.72329100 & 3.21533400 \\ \mathrm{H} & -7.33739900 & 0.14550200 & 3.05838400 \\ \mathrm{H} & -5.05896900 & -3.47922700 & 3.10725300 \\ \mathrm{H} & -6.87575000 & -2.08009700 & 4.05182100 \\ \mathrm{C} & -4.65086700 & 0.34318500 & -1.12172500 \\ \mathrm{C} & -5.86090900 & -0.13330600 & -1.63615500 \\ \mathrm{C} & -6.49178500 & 0.53079800 & -2.67863800 \\ \mathrm{C} & -5.92437500 & 1.67031500 & -3.23813300 \\ \mathrm{C} & -4.71741900 & 2.14326600 & -2.73483800 \\ \mathrm{C} & -4.08753700 & 1.49382000 & -1.68245500 \\ \mathrm{H} & -6.30351800 & -1.02517300 & -1.21554100 \\ \mathrm{H} & -7.42667000 & 0.14543200 & -3.06393500 \\ \mathrm{H} & -6.41508400 & 2.18192600 & -4.05467300 \\ \mathrm{H} & -4.26621500 & 3.03242700 & -3.15541300 \\ \mathrm{H} & -3.15399800 & 1.87223200 & -1.29085600 \\ \mathrm{~N} & -4.00745000 & -0.32938500 & -0.05155600\end{array}$

Table S22. Gas phase UB3LYP/cc-pVTZ (cc-pVTZ-PP Te) determined xyz coordinates (in $\AA$ ) for Benzo-Te(BPin)TPA at $\mathrm{T}_{1}$ equilibrium geometry.

$\begin{array}{crrr}\text { Te } & -4.42858800 & -0.80451800 & -0.04492800 \\ \mathrm{O} & -1.29948200 & 2.23387000 & 0.19580800 \\ \mathrm{O} & -3.58898200 & 2.16270000 & 0.14492200 \\ \mathrm{C} & -3.47776400 & -2.69535800 & -0.15455600 \\ \mathrm{C} & -2.06590700 & -2.54301900 & -0.21195900 \\ \mathrm{C} & -2.49129800 & -0.08138900 & -0.14500800 \\ \mathrm{C} & -1.75248500 & 3.54849200 & 0.61266300 \\ \mathrm{C} & -3.24161200 & 3.56736500 & 0.10150900 \\ \mathrm{~B} & -2.41108400 & 1.41877700 & 0.06075700 \\ \mathrm{C} & -4.10178400 & -3.91750300 & -0.22177400 \\ \mathrm{C} & -3.33416500 & -5.08494900 & -0.36735100 \\ \mathrm{C} & -1.95529000 & -4.97317200 & -0.47850200 \\ \mathrm{C} & -1.32908800 & -3.73477900 & -0.41742600 \\ \mathrm{H} & -5.18053900 & -3.99037800 & -0.17210400 \\ \mathrm{H} & -3.81795800 & -6.05063900 & -0.41393200 \\ \mathrm{H} & -1.35346600 & -5.85978800 & -0.62990700\end{array}$




\begin{tabular}{|c|c|c|c|}
\hline $\mathrm{H}$ & -0.26075600 & -3.68374900 & -0.56122200 \\
\hline $\mathrm{C}$ & -3.37462900 & 4.02099000 & -1.35451100 \\
\hline $\mathrm{H}$ & -4.38918000 & 3.81550200 & -1.69438100 \\
\hline $\mathrm{H}$ & -3.18663700 & 5.08922900 & -1.46407700 \\
\hline $\mathrm{H}$ & -2.68655000 & 3.47956700 & -2.00283000 \\
\hline $\mathrm{C}$ & -4.21877500 & 4.33928000 & 0.97842100 \\
\hline $\mathrm{H}$ & -3.94799000 & 5.39523800 & 1.02735400 \\
\hline $\mathrm{H}$ & -5.22084600 & 4.26876000 & 0.55543800 \\
\hline $\mathrm{H}$ & -4.25221400 & 3.94088600 & 1.98949500 \\
\hline $\mathrm{C}$ & -0.83973900 & 4.59590900 & -0.01097800 \\
\hline $\mathrm{H}$ & -1.18757800 & 5.60338300 & 0.22329100 \\
\hline $\mathrm{H}$ & 0.16772600 & 4.48589500 & 0.38981200 \\
\hline $\mathrm{H}$ & -0.78533800 & 4.48984400 & -1.09180400 \\
\hline $\mathrm{C}$ & -1.63780300 & 3.59605900 & 2.13797500 \\
\hline $\mathrm{H}$ & -0.60906200 & 3.37308700 & 2.41888100 \\
\hline $\mathrm{H}$ & -1.89521200 & 4.57991300 & 2.53072700 \\
\hline $\mathrm{H}$ & -2.28367400 & 2.85495100 & 2.60732600 \\
\hline $\mathrm{C}$ & -0.10399500 & -0.95630400 & -0.10244500 \\
\hline $\mathrm{C}$ & 0.78604800 & -1.76336300 & 0.64917900 \\
\hline $\mathrm{C}$ & 0.48003000 & 0.12283800 & -0.80657700 \\
\hline $\mathrm{C}$ & 2.14286800 & -1.52893200 & 0.67489400 \\
\hline $\mathrm{H}$ & 0.38805300 & -2.56615300 & 1.25192100 \\
\hline $\mathrm{C}$ & 1.83827000 & 0.34574100 & -0.80472900 \\
\hline $\mathrm{H}$ & -0.15294600 & 0.78351200 & -1.37595600 \\
\hline $\mathrm{C}$ & 2.70525000 & -0.47460700 & -0.06427400 \\
\hline $\mathrm{H}$ & 2.78204400 & -2.15950000 & 1.27620000 \\
\hline $\mathrm{H}$ & 2.24259900 & 1.17114700 & -1.37302300 \\
\hline $\mathrm{C}$ & -1.52251200 & -1.21354400 & -0.15555900 \\
\hline $\mathrm{C}$ & 4.75362800 & 0.21178900 & -1.22444100 \\
\hline $\mathrm{C}$ & 5.70711000 & 1.23173000 & -1.15492600 \\
\hline $\mathrm{C}$ & 4.46421600 & -0.36264300 & -2.46579600 \\
\hline $\mathrm{C}$ & 6.35824700 & 1.66069700 & -2.30284800 \\
\hline $\mathrm{H}$ & 5.93299600 & 1.68509200 & -0.20021500 \\
\hline $\mathrm{C}$ & 5.11067100 & 0.08119900 & -3.61045800 \\
\hline $\mathrm{H}$ & 3.73365600 & -1.15664000 & -2.52654600 \\
\hline $\mathrm{C}$ & 6.06301100 & 1.09217100 & -3.53712000 \\
\hline $\mathrm{H}$ & 7.09180700 & 2.45289200 & -2.23231500 \\
\hline $\mathrm{H}$ & 4.87668300 & -0.37561200 & -4.56291000 \\
\hline $\mathrm{H}$ & 6.56864100 & 1.43222700 & -4.43039200 \\
\hline $\mathrm{C}$ & 4.84427800 & -0.43911400 & 1.13721800 \\
\hline $\mathrm{C}$ & 6.08675000 & -1.07834000 & 1.09031500 \\
\hline $\mathrm{C}$ & 6.82286200 & -1.26362000 & 2.25188500 \\
\hline $\mathrm{C}$ & 6.33074700 & -0.82848800 & 3.47762400 \\
\hline $\mathrm{C}$ & 5.09242800 & -0.19747600 & 3.52846000 \\
\hline $\mathrm{C}$ & 4.35552600 & 0.00360500 & 2.37003500 \\
\hline $\mathrm{H}$ & 6.46996300 & -1.42812700 & 0.14222600 \\
\hline $\mathrm{H}$ & 7.78160700 & -1.76225000 & 2.19866100 \\
\hline $\mathrm{H}$ & 6.90493200 & -0.97899600 & 4.38143100 \\
\hline $\mathrm{H}$ & 4.70076000 & 0.15342300 & 4.47401300 \\
\hline $\mathrm{H}$ & 3.39926100 & 0.50524400 & 2.41351700 \\
\hline $\mathrm{N}$ & 4.09262000 & -0.23814900 & -0.05080900 \\
\hline
\end{tabular}


Table S23. Gas phase B3LYP/cc-pVTZ (cc-pVTZ-PP Te) determined xyz coordinates (in $\AA$ ) for $\mathrm{TeC}_{4}(2 \text {-thienyl })_{4}$ at $\mathrm{S}_{0}$ equilibrium geometry.

\begin{tabular}{cccc} 
Te & 0.00357100 & -2.40715800 & 0.00656000 \\
$\mathrm{C}$ & -1.38427100 & -0.83887100 & -0.03230000 \\
$\mathrm{C}$ & 1.37513100 & -0.82854600 & -0.02582300 \\
$\mathrm{C}$ & -0.73537300 & 0.36990000 & -0.11321400 \\
$\mathrm{C}$ & 0.71969300 & 0.37621000 & -0.09920000 \\
$\mathrm{C}$ & -2.79586200 & -1.15585800 & 0.01711100 \\
$\mathrm{C}$ & -3.36453200 & -2.29364000 & 0.54873700 \\
$\mathrm{~S}$ & -4.05539200 & -0.14518800 & -0.66553500 \\
$\mathrm{C}$ & -4.77061200 & -2.35783900 & 0.41413500 \\
$\mathrm{H}$ & -2.78240200 & -3.05404000 & 1.04969800 \\
$\mathrm{C}$ & -5.28401400 & -1.26950700 & -0.22706200 \\
$\mathrm{H}$ & -5.37284700 & -3.17366100 & 0.78496500 \\
$\mathrm{H}$ & -6.31392300 & -1.05418900 & -0.45709400 \\
$\mathrm{C}$ & -1.48493600 & 1.64300500 & -0.17560900 \\
$\mathrm{C}$ & -1.85551900 & 2.36050200 & -1.27916100 \\
$\mathrm{~S}$ & -2.02322600 & 2.44921300 & 1.27166400 \\
$\mathrm{C}$ & -2.57387000 & 3.54661500 & -0.97291400 \\
$\mathrm{H}$ & -1.62645600 & 2.03882900 & -2.28448200 \\
$\mathrm{C}$ & -2.74298100 & 3.72645700 & 0.36748500 \\
$\mathrm{H}$ & -2.94840700 & 4.23286100 & -1.71783500 \\
$\mathrm{H}$ & -3.24422500 & 4.53337800 & 0.87475900 \\
$\mathrm{C}$ & 1.46239300 & 1.65432400 & -0.10363600 \\
$\mathrm{C}$ & 1.70293300 & 2.50993200 & -1.14276000 \\
$\mathrm{~S}$ & 2.17628200 & 2.27471300 & 1.36033100 \\
$\mathrm{C}$ & 2.46483400 & 3.65088600 & -0.77639700 \\
$\mathrm{H}$ & 1.35219400 & 2.31970500 & -2.14602700 \\
$\mathrm{C}$ & 2.79473700 & 3.66042500 & 0.54616800 \\
$\mathrm{H}$ & 2.75447600 & 4.42807000 & -1.46792100 \\
$\mathrm{H}$ & 3.36047200 & 4.39950500 & 1.08777200 \\
$\mathrm{C}$ & 2.79096300 & -1.13186300 & -0.00868300 \\
$\mathrm{C}$ & 3.40187600 & -2.20701600 & 0.59816600 \\
$\mathrm{~S}$ & 3.99028200 & -0.19070600 & -0.87157600 \\
$\mathrm{C}$ & 4.79882100 & -2.27472800 & 0.38304200 \\
$\mathrm{H}$ & 2.85761800 & -2.91545100 & 1.20616800 \\
$\mathrm{C}$ & 5.25841700 & -1.25532200 & -0.39734400 \\
$\mathrm{H}$ & 5.43397300 & -3.04338700 & 0.79722600 \\
$\mathrm{H}$ & 6.26972100 & -1.05830200 & -0.71092600 \\
& & & \\
\hline
\end{tabular}


Table S24. Gas phase B3LYP/cc-pVTZ (cc-pVTZ-PP Te) determined xyz coordinates (in Å) for Naph-Te-6-Naph at $S_{0}$ equilibrium geometry.

$\begin{array}{lrrr}\mathrm{Te} & 0.00003100 & -1.04379700 & -0.43455000 \\ \mathrm{C} & -0.72330000 & 1.69762800 & -0.01229800 \\ \mathrm{C} & 0.72326500 & 1.69765500 & -0.01227600 \\ \mathrm{C} & 0.77488200 & 3.68733700 & 1.51976100 \\ \mathrm{H} & 1.15584800 & 4.70682300 & 1.59892700 \\ \mathrm{H} & 1.13997600 & 3.15438100 & 2.39874700 \\ \mathrm{C} & -0.77509400 & 3.68759000 & 1.51951800 \\ \mathrm{H} & -1.14063800 & 3.15512200 & 2.39861100 \\ \mathrm{H} & -1.15574600 & 4.70723600 & 1.59814300 \\ \mathrm{C} & -1.38358400 & 3.02555600 & 0.26821100 \\ \mathrm{H} & -1.24200900 & 3.68831000 & -0.59278000 \\ \mathrm{H} & -2.45762300 & 2.91126700 & 0.39760100 \\ \mathrm{C} & 1.38352500 & 3.02555200 & 0.26840800 \\ \mathrm{H} & 2.45754800 & 2.91121900 & 0.39790600 \\ \mathrm{H} & 1.24206300 & 3.68844900 & -0.59249200 \\ \mathrm{C} & 4.81462900 & -0.98953000 & 0.40115500 \\ \mathrm{C} & 2.82546500 & 0.26610700 & -0.26071700 \\ \mathrm{C} & 4.98828100 & 0.81623200 & -1.23100000 \\ \mathrm{H} & 5.58831500 & 1.41225100 & -1.90745500 \\ \mathrm{C} & -4.81462900 & -0.98952600 & 0.40119400 \\ \mathrm{C} & -4.98822900 & 0.81602700 & -1.23120200 \\ \mathrm{H} & -5.58824400 & 1.41194900 & -1.90775900 \\ \mathrm{C} & 1.37979400 & 0.51489300 & -0.18899700 \\ \mathrm{C} & -1.37978400 & 0.51484900 & -0.18901700 \\ \mathrm{C} & 5.61963600 & -0.19734200 & -0.47091900 \\ \mathrm{C} & -5.61960200 & -0.19744400 & -0.47100700 \\ \mathrm{C} & -3.64256100 & 1.04002500 & -1.13347600 \\ \mathrm{H} & -3.17731100 & 1.80070300 & -1.74442600 \\ \mathrm{C} & -2.82545500 & 0.26604600 & -0.26076000 \\ \mathrm{C} & -3.42708200 & -0.73263100 & 0.47909000 \\ \mathrm{H} & -2.82770600 & -1.33058900 & 1.15457000 \\ \mathrm{C} & 3.64261300 & 1.04021700 & -1.13329000 \\ \mathrm{H} & 3.17738200 & 1.80097900 & -1.74414800 \\ \mathrm{C} & 3.42708300 & -0.73264600 & 0.47904400 \\ \mathrm{H} & 2.82768400 & -1.33066900 & 1.15444600 \\ \mathrm{C} & 7.58010100 & -1.44911600 & 0.20439900 \\ \mathrm{H} & 8.64334700 & -1.63725000 & 0.13790100 \\ \mathrm{C} & 6.78588800 & -2.23167100 & 1.07019500 \\ \mathrm{H} & 7.24840000 & -3.01206300 & 1.65974300 \\ \mathrm{C} & 5.43737000 & -2.00741500 & 1.16554500 \\ \mathrm{H} & 4.82793800 & -2.60795800 & 1.82913400 \\ \mathrm{H} & 7.00837100 & -0.45583800 & -0.54782500 \\ & 7.61525100 & 0.14609500 & -1.21265400 \\ \mathrm{H} & -7.00834600 & -0.45593700 & -0.54791100 \\ -7.61519900 & 0.14590900 & -1.21284300\end{array}$




$\begin{array}{llll}\mathrm{C} & -6.78591900 & -2.23154300 & 1.07036700 \\ \mathrm{H} & -7.24845700 & -3.01184000 & 1.66002100 \\ \mathrm{C} & -5.43740000 & -2.00729700 & 1.16571000 \\ \mathrm{H} & -4.82799000 & -2.60775500 & 1.82939600 \\ \mathrm{C} & -7.58010300 & -1.44909600 & 0.20444200 \\ \mathrm{H} & -8.64335100 & -1.63722900 & 0.13795200\end{array}$

Table S25. Gas phase B3LYP/cc-pVTZ (cc-pVTZ-PP Te) determined xyz coordinates (in $\AA$ ) for Naph-Te-6-Naph at $T_{1}$ equilibrium geometry.

$\begin{array}{lrrr}\mathrm{Te} & -0.00000300 & -0.91127700 & 0.50863700 \\ \mathrm{C} & -0.68536500 & 1.90334700 & -0.01414800 \\ \mathrm{C} & 0.68537300 & 1.90334600 & -0.01413200 \\ \mathrm{C} & 0.77137200 & 4.11870400 & 1.18095000 \\ \mathrm{H} & 1.15760100 & 5.13394500 & 1.07576200 \\ \mathrm{H} & 1.13772500 & 3.74755200 & 2.13863500 \\ \mathrm{C} & -0.77143000 & 4.11888000 & 1.18074300 \\ \mathrm{H} & -1.13812000 & 3.74811900 & 2.13845000 \\ \mathrm{H} & -1.15739500 & 5.13417800 & 1.07514000 \\ \mathrm{C} & -1.37972500 & 3.24216700 & 0.06596100 \\ \mathrm{H} & -1.28597000 & 3.76474700 & -0.89320600 \\ \mathrm{H} & -2.44267900 & 3.12555600 & 0.25449700 \\ \mathrm{C} & 1.37973500 & 3.24215800 & 0.06608800 \\ \mathrm{H} & 2.44268100 & 3.12553000 & 0.25466100 \\ \mathrm{H} & 1.28601500 & 3.76484100 & -0.89302600 \\ \mathrm{C} & 4.67187100 & -1.19366700 & 0.03763200 \\ \mathrm{C} & 2.74543300 & 0.33782200 & -0.19102300 \\ \mathrm{C} & 4.88860400 & 0.88141700 & -1.23879600 \\ \mathrm{H} & 5.48676800 & 1.53738600 & -1.85937200 \\ \mathrm{C} & -4.67186700 & -1.19365900 & 0.03765500 \\ \mathrm{C} & -4.88859300 & 0.88137800 & -1.23884900 \\ \mathrm{H} & -5.48675200 & 1.53732400 & -1.85945500 \\ \mathrm{C} & 1.38711800 & 0.63638300 & 0.05845900 \\ \mathrm{C} & -1.38711100 & 0.63638900 & 0.05843900 \\ \mathrm{C} & 5.48771400 & -0.30883600 & -0.73331300 \\ \mathrm{C} & -5.48770400 & -0.30885700 & -0.73332900 \\ \mathrm{C} & -3.59153600 & 1.19613500 & -0.98170900 \\ \mathrm{H} & -3.17006100 & 2.08317000 & -1.42717300 \\ \mathrm{C} & -2.74543000 & 0.33782300 & -0.19104000 \\ \mathrm{C} & -3.32982300 & -0.85586100 & 0.27865600 \\ \mathrm{H} & -2.73932600 & -1.53351200 & 0.88376300 \\ \mathrm{C} & 3.59154600 & 1.19616300 & -0.98165500 \\ \mathrm{H} & 3.17007200 & 2.08321400 & -1.42708900 \\ \mathrm{C} & 3.32982500 & -0.85588000 & 0.27863500 \\ \mathrm{H} & 2.73932300 & -1.53355400 & 0.88371200 \\ \mathrm{C} & 7.36725400 & -1.81346100 & -0.47444600 \\ \mathrm{H} & 8.40253500 & -2.06075300 & -0.66644400 \\ \mathrm{C} & 6.56898900 & -2.68802500 & 0.28736300\end{array}$




$\begin{array}{cccc}\mathrm{H} & 6.99798100 & -3.60192800 & 0.67632000 \\ \mathrm{C} & 5.25334300 & -2.38769000 & 0.53740200 \\ \mathrm{H} & 4.64041700 & -3.06131500 & 1.12294600 \\ \mathrm{C} & 6.83057600 & -0.64662200 & -0.97439000 \\ \mathrm{H} & 7.44248300 & 0.02672400 & -1.56173600 \\ \mathrm{C} & -6.83056400 & -0.64665600 & -0.97440700 \\ \mathrm{H} & -7.44246800 & 0.02666800 & -1.56178300 \\ \mathrm{C} & -6.56898400 & -2.68801200 & 0.28742300 \\ \mathrm{H} & -6.99797700 & -3.60190200 & 0.67640900 \\ \mathrm{C} & -5.25334100 & -2.38766400 & 0.53746300 \\ \mathrm{H} & -4.64041900 & -3.06126700 & 1.12303700 \\ \mathrm{C} & -7.36724300 & -1.81347700 & -0.47442600 \\ \mathrm{H} & -8.40252300 & -2.06077900 & -0.66642400\end{array}$

Table S26. Gas phase B3LYP/cc-pVTZ (cc-pVTZ-PP Te) determined xyz coordinates (in $\AA$ ) for Fl-Te-6-Fl at $\mathrm{S}_{0}$ equilibrium geometry.

$\begin{array}{crrr}\mathrm{Te} & 0.00019700 & -1.36317900 & -0.23461500 \\ \mathrm{C} & 0.72332300 & 1.40868100 & -0.35049700 \\ \mathrm{C} & -0.72359000 & 1.40850100 & -0.35053200 \\ \mathrm{C} & 0.77457500 & 3.55251600 & -1.66025700 \\ \mathrm{H} & 1.13985500 & 3.11713500 & -2.59139700 \\ \mathrm{H} & 1.15527100 & 4.57476100 & -1.62920300 \\ \mathrm{C} & -0.77541400 & 3.55213900 & -1.66041500 \\ \mathrm{H} & -1.14031000 & 3.11628200 & -2.59148400 \\ \mathrm{H} & -1.15663500 & 4.57419900 & -1.62974700 \\ \mathrm{C} & -1.38336300 & 2.75908700 & -0.48749800 \\ \mathrm{H} & -2.45732700 & 2.65805400 & -0.62860400 \\ \mathrm{H} & -1.24339400 & 3.32617100 & 0.43967600 \\ \mathrm{C} & 1.38272100 & 2.75945300 & -0.48743400 \\ \mathrm{H} & 1.24260300 & 3.32639000 & 0.43981000 \\ \mathrm{H} & 2.45671000 & 2.65874200 & -0.62857500 \\ \mathrm{C} & 1.38181500 & 0.21450900 & -0.30174700 \\ \mathrm{C} & -1.38177700 & 0.21414000 & -0.30166900 \\ \mathrm{C} & -4.99968100 & 0.37057000 & 0.71412300 \\ \mathrm{C} & -5.58012400 & -0.56693000 & -0.15563500 \\ \mathrm{C} & -6.05841100 & 0.96072600 & 1.61727500 \\ \mathrm{C} & -3.42149600 & -0.98384800 & -1.11016700 \\ \mathrm{C} & -7.01635700 & -0.63563100 & 0.11800000 \\ \mathrm{C} & -4.78266600 & -1.24841700 & -1.06995400 \\ \mathrm{C} & -7.31623300 & 0.25880800 & 1.16011200 \\ \mathrm{H} & -6.13222500 & 2.04623800 & 1.50493200 \\ \mathrm{H} & -5.84551500 & 0.77048500 & 2.67316000 \\ \mathrm{H} & -2.79925600 & -1.49997900 & -1.82941400 \\ \mathrm{C} & -8.02357400 & -1.39876700 & -0.46563500 \\ \mathrm{H} & -5.21037600 & -1.97546400 & -1.74799200\end{array}$




$\begin{array}{cccc}\mathrm{C} & -8.61591000 & 0.39220000 & 1.61862300 \\ \mathrm{C} & -9.32670700 & -1.26006100 & -0.00120400 \\ \mathrm{H} & -7.80213000 & -2.09004000 & -1.26837700 \\ \mathrm{C} & -9.62262500 & -0.37284100 & 1.03195300 \\ \mathrm{H} & -8.85328600 & 1.07935800 & 2.42125300 \\ \mathrm{H} & -10.11997700 & -1.84640900 & -0.44564300 \\ \mathrm{H} & -10.64214600 & -0.27764900 & 1.38105300 \\ \mathrm{C} & 4.99973600 & 0.37064300 & 0.71422200 \\ \mathrm{C} & 2.82603600 & -0.04211300 & -0.25347400 \\ \mathrm{C} & 5.58017400 & -0.56660300 & -0.15578800 \\ \mathrm{C} & 6.05844300 & 0.96048800 & 1.61759700 \\ \mathrm{C} & 3.42155300 & -0.98320100 & -1.11047400 \\ \mathrm{C} & 7.01639700 & -0.63544200 & 0.11787300 \\ \mathrm{C} & 4.78270700 & -1.24781900 & -1.07031700 \\ \mathrm{C} & 7.31626100 & 0.25867300 & 1.16026400 \\ \mathrm{H} & 5.84550100 & 0.76992600 & 2.67341500 \\ \mathrm{H} & 6.13229600 & 2.04603700 & 1.50560400 \\ \mathrm{H} & 2.79932200 & -1.49913300 & -1.82987400 \\ \mathrm{C} & 8.02361200 & -1.39843500 & -0.46595000 \\ \mathrm{H} & 5.21041000 & -1.97469300 & -1.74854500 \\ \mathrm{C} & 8.61592300 & 0.39188000 & 1.61887700 \\ \mathrm{C} & 9.32673000 & -1.25991300 & -0.00142000 \\ \mathrm{H} & 7.80218900 & -2.08945300 & -1.26891800 \\ \mathrm{C} & 9.62263500 & -0.37302000 & 1.03202300 \\ \mathrm{H} & 8.85328400 & 1.07878500 & 2.42172700 \\ \mathrm{H} & 10.11999900 & -1.84615200 & -0.44600500 \\ \mathrm{H} & 10.64214400 & -0.27797600 & 1.38119700 \\ \mathrm{C} & 3.64288100 & 0.62956900 & 0.67251600 \\ \mathrm{H} & 3.19474100 & 1.33059400 & 1.36405200 \\ \mathrm{C} & -2.82599000 & -0.04256800 & -0.25340500 \\ \mathrm{C} & -3.64283200 & 0.62943500 & 0.67238100 \\ \mathrm{H} & -3.19461700 & 1.33064400 & 1.36368400 \\ & & & \\ & & & \\ & & & \end{array}$

Table S27. Gas phase B3LYP/cc-pVTZ (cc-pVTZ-PP Te) determined xyz coordinates (in $\AA$ ) for $\mathbf{A r}^{\mathrm{F}}$-Te-6-Ar ${ }^{\mathrm{F}}$ at $\mathrm{S}_{0}$ equilibrium geometry.

$\begin{array}{llll}\mathrm{Te} & 0.00001 & -0.97304 & -0.72581 \\ \mathrm{C} & -0.72301 & 1.25383 & 0.93931 \\ \mathrm{C} & 0.72303 & 1.25383 & 0.93931 \\ \mathrm{C} & 0.77477 & 2.29975 & 3.22341 \\ \mathrm{H} & 1.15619 & 3.16623 & 3.76487 \\ \mathrm{H} & 1.1403 & 1.42048 & 3.75523 \\ \mathrm{C} & -0.77472 & 2.29977 & 3.22342 \\ \mathrm{H} & -1.14027 & 1.42054 & 3.75528 \\ \mathrm{H} & -1.15611 & 3.1663 & 3.76484\end{array}$




$\begin{array}{lccl}\text { C } & -1.38374 & 2.29569 & 1.8081 \\ \text { H } & -1.24449 & 3.28214 & 1.35275 \\ \text { H } & -2.45785 & 2.13579 & 1.87164 \\ \text { C } & 1.38376 & 2.2957 & 1.80808 \\ \text { H } & 2.45787 & 2.13582 & 1.8716 \\ \text { H } & 1.24448 & 3.28215 & 1.35275 \\ \text { C } & 4.78413 & -1.31704 & 0.08738 \\ \text { C } & 2.82026 & 0.1053 & 0.05062 \\ \text { C } & 5.01164 & 0.99281 & -0.48925 \\ \text { C } & -5.01165 & 0.99281 & -0.48908 \\ \text { C } & -2.82024 & 0.10528 & 0.05061 \\ \text { C } & -4.78406 & -1.31712 & 0.08717 \\ \text { C } & 1.37403 & 0.2917 & 0.22337 \\ \text { C } & -1.37401 & 0.2917 & 0.22338 \\ \text { C } & 5.59445 & -0.24871 & -0.28184 \\ \text { H } & 6.65891 & -0.38095 & -0.39647 \\ \text { C } & -5.59442 & -0.24876 & -0.28186 \\ \text { H } & -6.65889 & -0.38101 & -0.3965 \\ \text { C } & 3.41752 & -1.14613 & 0.25063 \\ \text { H } & 2.80768 & -1.9826 & 0.56037 \\ \text { C } & 3.6416 & 1.16967 & -0.33367 \\ \text { H } & 3.20435 & 2.13915 & -0.52164 \\ \text { C } & -3.41747 & -1.1462 & 0.25043 \\ \text { H } & -2.8076 & -1.98271 & 0.56003 \\ \text { C } & -3.64161 & 1.16968 & -0.3335 \\ \text { H } & -3.2044 & 2.13921 & -0.52134 \\ \text { C } & -5.3982 & -2.68128 & 0.27158 \\ \text { C } & -5.84939 & 2.16544 & -0.93024 \\ \text { C } & 5.39821 & -2.68119 & 0.272 \\ \text { C } & 5.84935 & 2.16537 & -0.93065 \\ \text { F } & -5.6719 & 2.43384 & -2.24177 \\ \text { F } & -7.16409 & 1.95342 & -0.74401 \\ \text { F } & -5.52442 & 3.2897 & -0.25813 \\ \text { F } & -5.47172 & -3.35055 & -0.89987 \\ \text { F } & -6.65197 & -2.60687 & 0.75738 \\ \text { F } & -4.68401 & -3.44918 & 1.11615 \\ \text { F } & 5.47046 & -3.35118 & -0.89911 \\ \text { F } & 4.68466 & -3.44845 & 1.11771 \\ \text { F } & 6.65245 & -2.60675 & 0.75657 \\ \text { F } & 7.16404 & 1.95351 & -0.74414 \\ \text { F } & 5.67205 & 2.43335 & -2.24229 \\ \text { F } & 5.5242 & 3.28982 & -0.25894 \\ & & & \end{array}$


Table S28. Gas phase UB3LYP/cc-pVTZ (cc-pVTZ-PP Te) determined xyz coordinates (in $\AA$ ) for $\mathbf{A r}^{\mathrm{F}}$-Te-6-Ar ${ }^{\mathrm{F}}$ at $\mathrm{T}_{1}$ equilibrium geometry.

$\begin{array}{cccc}\text { Te } & 0.00002 & 1.3299 & 0.54122 \\ \mathrm{C} & 0.6832 & -1.51305 & 0.96675 \\ \mathrm{C} & -0.68321 & -1.51304 & 0.96674 \\ \mathrm{C} & -0.77139 & -3.19729 & 2.83507 \\ \mathrm{H} & -1.15793 & -4.18929 & 3.07176 \\ \mathrm{H} & -1.13697 & -2.52992 & 3.61585 \\ \mathrm{C} & 0.7714 & -3.19787 & 2.8348 \\ \mathrm{H} & 1.13772 & -2.53141 & 3.61602 \\ \mathrm{H} & 1.15726 & -4.19035 & 3.07059 \\ \mathrm{C} & 1.38338 & -2.74227 & 1.49256 \\ \mathrm{H} & 1.29537 & -3.55571 & 0.76349 \\ \mathrm{H} & 2.44544 & -2.56527 & 1.63503 \\ \mathrm{C} & -1.38342 & -2.74219 & 1.49271 \\ \mathrm{H} & -2.44548 & -2.56516 & 1.63513 \\ \mathrm{H} & -1.29535 & -3.55582 & 0.76384 \\ \mathrm{C} & -4.63663 & 1.35446 & -0.20234 \\ \mathrm{C} & -2.72643 & -0.11635 & 0.19374 \\ \mathrm{C} & -4.83033 & -0.98798 & -0.68329 \\ \mathrm{C} & 4.8303 & -0.98797 & -0.68338 \\ \mathrm{C} & 2.72644 & -0.11637 & 0.19376 \\ \mathrm{C} & 4.63664 & 1.35444 & -0.20233 \\ \mathrm{C} & -1.37861 & -0.29061 & 0.59474 \\ \mathrm{C} & 1.37862 & -0.29062 & 0.59477 \\ \mathrm{C} & -5.40971 & 0.28143 & -0.64502 \\ \mathrm{H} & -6.43112 & 0.43144 & -0.96093 \\ \mathrm{C} & 5.40969 & 0.28143 & -0.64509 \\ \mathrm{H} & 6.43108 & 0.43145 & -0.96107 \\ \mathrm{C} & -3.33062 & 1.16958 & 0.19987 \\ \mathrm{H} & -2.76789 & 2.02345 & 0.54873 \\ \mathrm{C} & -3.52732 & -1.19178 & -0.27956 \\ \mathrm{H} & -3.10501 & -2.17816 & -0.36555 \\ \mathrm{C} & 3.33064 & 1.16955 & 0.19992 \\ \mathrm{H} & 2.76792 & 2.0234 & 0.54883 \\ \mathrm{C} & 3.52731 & -1.19179 & -0.2796 \\ \mathrm{H} & 3.105 & -2.17817 & -0.36562 \\ \mathrm{C} & 5.27153 & 2.72026 & -0.13459 \\ \mathrm{~F} & 5.64764 & -2.13262 & -1.22581 \\ \mathrm{~F} & -5.27153 & 2.72028 & -0.13474 \\ \mathrm{C} & -5.64765 & -2.13266 & -1.22567 \\ \mathrm{C} & 5.83277 & -2.02089 & -2.55818 \\ \mathrm{~F} & 6.87367 & -2.1737 & -0.66587 \\ \mathrm{~F} & 5.06837 & -3.32829 & -1.00657 \\ \mathrm{~F} & & 3.01117 & -1.2659 \\ \mathrm{~F} & 2.79873 & 0.87766 \\ \mathrm{H} & 3.69818 & 0.05765\end{array}$




\begin{tabular}{|c|c|c|c|}
\hline $\mathrm{F}$ & -5.94286 & 3.01141 & -1.26644 \\
\hline $\mathrm{F}$ & -4.36743 & 3.69815 & 0.05827 \\
\hline $\mathrm{F}$ & -6.16241 & 2.79856 & 0.87694 \\
\hline $\mathrm{F}$ & -6.87386 & -2.17344 & -0.66611 \\
\hline $\mathrm{F}$ & -5.83236 & -2.02124 & -2.55813 \\
\hline $\mathrm{F}$ & -5.0686 & -3.32834 & -1.00595 \\
\hline
\end{tabular}

Table S29. B3LYP/cc-pVTZ determined $x y z$ coordinates (in $\AA$ ) for $4 \mathbf{B S}$ in THF at $\mathrm{S}_{0}$ equilibrium geometry.

$\begin{array}{cccc}\mathrm{O} & -3.79617 & -0.69918 & 0.56079 \\ \mathrm{O} & -3.24121 & -2.7145 & -0.37902 \\ \mathrm{~B} & -2.80071 & -1.48489 & 0.0423 \\ \mathrm{O} & 3.71907 & -0.79702 & -0.35219 \\ \mathrm{O} & 3.04829 & -2.91566 & 0.21245 \\ \mathrm{~B} & 2.66867 & -1.62133 & -0.03799 \\ \mathrm{C} & 1.19965 & -1.14368 & 0.00496 \\ \mathrm{C} & -1.31068 & -1.06621 & -0.02008 \\ \mathrm{C} & -4.64155 & -2.82098 & -0.0429 \\ \mathrm{C} & -5.04768 & -1.39631 & 0.37984 \\ \mathrm{C} & 4.46769 & -3.01485 & -0.03559 \\ \mathrm{C} & 4.93709 & -1.55593 & -0.19201 \\ \mathrm{C} & 0.70101 & 0.14759 & 0.01486 \\ \mathrm{C} & -0.73227 & 0.19357 & -0.00887 \\ \mathrm{~B} & 1.60325 & 1.42581 & 0.04573 \\ \mathrm{O} & 2.41266 & 1.76257 & 1.09828 \\ \mathrm{O} & 1.64063 & 2.33567 & -0.98088 \\ \mathrm{C} & 3.15538 & 2.94226 & 0.72481 \\ \mathrm{C} & 2.47206 & 3.43964 & -0.56223 \\ \mathrm{~B} & -1.52182 & 1.53683 & -0.04809 \\ \mathrm{O} & -1.19272 & 2.60388 & 0.75272 \\ \mathrm{O} & -2.5583 & 1.80435 & -0.90284 \\ \mathrm{C} & -2.00375 & 3.72742 & 0.3464 \\ \mathrm{C} & -3.06705 & 3.11679 & -0.58417 \\ \mathrm{H} & -1.36577 & 4.44629 & -0.16815 \\ \mathrm{H} & -2.43122 & 4.19759 & 1.22936 \\ \mathrm{H} & -4.03312 & 3.00349 & -0.09167 \\ \mathrm{H} & -3.20011 & 3.68131 & -1.50457 \\ \mathrm{H} & 1.83426 & 4.30589 & -0.38564 \\ \mathrm{H} & 3.10869 & 3.66541 & 1.53632 \\ \mathrm{H} & 4.19463 & 2.65814 & 0.55863 \\ \mathrm{H} & 3.17959 & 3.67395 & -1.35475 \\ \mathrm{H} & 5.45893 & -1.19078 & 0.69253 \\ \mathrm{H} & 5.56776 & -1.40539 & -1.06547 \\ \mathrm{H} & 4.62082 & -3.60026 & -0.94216 \\ \mathrm{H} & 4.94051 & -3.52413 & 0.80133 \\ \mathrm{H} & -4.74958 & -3.54209 & 0.76731 \\ \mathrm{H} & -5.19013 & -3.17728 & -0.91205\end{array}$




$$
\begin{aligned}
& \begin{array}{llll}
\mathrm{H} & -5.6224 & -0.88251 & -0.39079
\end{array}
\end{aligned}
$$

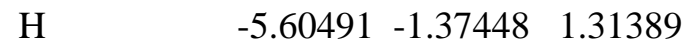

$$
\begin{aligned}
& \text { S } \quad \begin{array}{llll}
-0.09326 & -2.29212 & -0.01991
\end{array}
\end{aligned}
$$

Table S30. B3LYP/cc-pVTZ determined $x y z$ coordinates (in $\AA$ ) for 4 BSe in THF at $S_{0}$ equilibrium geometry.

$\begin{array}{llll}\mathrm{O} & -3.8215 & -0.40687 & 0.58918 \\ \mathrm{O} & -3.35088 & -2.42543 & -0.38875 \\ \mathrm{~B} & -2.85902 & -1.21887 & 0.04639 \\ \mathrm{O} & 3.76478 & -0.53456 & -0.35465 \\ \mathrm{O} & 3.15424 & -2.66912 & 0.21757 \\ \mathrm{~B} & 2.73642 & -1.38513 & -0.03298 \\ \mathrm{C} & 1.25467 & -0.95536 & 0.01298 \\ \mathrm{C} & -1.35537 & -0.86216 & -0.02238 \\ \mathrm{C} & -4.74956 & -2.48673 & -0.03636 \\ \mathrm{C} & -5.09933 & -1.0543 & 0.40862 \\ \mathrm{C} & 4.57338 & -2.7309 & -0.04099 \\ \mathrm{C} & 5.00307 & -1.26014 & -0.19747 \\ \mathrm{C} & 0.71416 & 0.31225 & 0.01894 \\ \mathrm{C} & -0.72506 & 0.36537 & -0.01204 \\ \mathrm{~B} & 1.60147 & 1.60358 & 0.05507 \\ \mathrm{O} & 2.38771 & 1.95692 & 1.11958 \\ \mathrm{O} & 1.64861 & 2.50546 & -0.97828 \\ \mathrm{C} & 3.12371 & 3.14237 & 0.75155 \\ \mathrm{C} & 2.46298 & 3.62015 & -0.5548 \\ \mathrm{~B} & -1.48868 & 1.72584 & -0.06032 \\ \mathrm{O} & -1.16133 & 2.7835 & 0.75368 \\ \mathrm{O} & -2.50108 & 2.01463 & -0.9371 \\ \mathrm{C} & -1.94525 & 3.92173 & 0.33469 \\ \mathrm{C} & -2.99875 & 3.33147 & -0.61939 \\ \mathrm{H} & -1.2852 & 4.63144 & -0.16456 \\ \mathrm{H} & -2.3828 & 4.3962 & 1.21035 \\ \mathrm{H} & -3.97515 & 3.22764 & -0.14541 \\ \mathrm{H} & -3.1073 & 3.90365 & -1.53826 \\ \mathrm{H} & 1.81564 & 4.48367 & -0.40109 \\ \mathrm{H} & 3.05022 & 3.87212 & 1.5552 \\ \mathrm{H} & 4.16984 & 2.86999 & 0.61086 \\ \mathrm{H} & 3.1849 & 3.85107 & -1.33532 \\ \mathrm{H} & 5.51675 & -0.88188 & 0.68644 \\ \mathrm{H} & 5.62798 & -1.09218 & -1.07195 \\ \mathrm{H} & 4.73517 & -3.30989 & -0.95031 \\ \mathrm{H} & 5.06606 & -3.22955 & 0.79092 \\ \mathrm{H} & -4.8733 & -3.21346 & 0.7667 \\ \mathrm{H} & -5.32141 & -2.81283 & -0.90243 \\ \mathrm{H} & -5.6602 & -0.5092 & -0.35074 \\ \mathrm{H} & -5.6489 & -1.02458 & 1.34703 \\ \mathrm{Se} & -0.0994 & -2.25436 & -0.017 \\ & & & \end{array}$


Table S31. B3LYP/cc-pVTZ determined xyz coordinates (in $\AA$ ) for 4BTe in THF at $S_{0}$ equilibrium geometry.

\begin{tabular}{lcll} 
Te & -0.10212 & -2.28783 & -0.01582 \\
$\mathrm{O}$ & -3.8345 & -0.09284 & 0.6503 \\
$\mathrm{O}$ & -3.50326 & -2.08982 & -0.42336 \\
$\mathrm{~B}$ & -2.92938 & -0.93355 & 0.05151 \\
$\mathrm{O}$ & 3.81874 & -0.2301 & -0.34671 \\
$\mathrm{O}$ & 3.30765 & -2.39107 & 0.2207 \\
$\mathrm{~B}$ & 2.82781 & -1.12595 & -0.02502 \\
$\mathrm{C}$ & 1.32688 & -0.7771 & 0.02035 \\
$\mathrm{C}$ & -1.40879 & -0.67183 & -0.02596 \\
$\mathrm{C}$ & -4.89568 & -2.08408 & -0.04422 \\
$\mathrm{C}$ & -5.15143 & -0.65336 & 0.46472 \\
$\mathrm{C}$ & 4.72583 & -2.38847 & -0.04496 \\
$\mathrm{C}$ & 5.08859 & -0.8993 & -0.19549 \\
$\mathrm{C}$ & 0.72895 & 0.46178 & 0.0217 \\
$\mathrm{C}$ & -0.71666 & 0.519 & -0.017 \\
$\mathrm{~B}$ & 1.59192 & 1.77366 & 0.06392 \\
$\mathrm{O}$ & 2.33324 & 2.16018 & 1.14869 \\
$\mathrm{O}$ & 1.66023 & 2.65723 & -0.98401 \\
$\mathrm{C}$ & 3.05508 & 3.35664 & 0.79009 \\
$\mathrm{C}$ & 2.44885 & 3.78929 & -0.5587 \\
$\mathrm{~B}$ & -1.45218 & 1.89802 & -0.07837 \\
$\mathrm{O}$ & -1.13064 & 2.94785 & 0.74813 \\
$\mathrm{O}$ & -2.43697 & 2.2061 & -0.98039 \\
$\mathrm{C}$ & -1.88718 & 4.09888 & 0.31344 \\
$\mathrm{C}$ & -2.92765 & 3.52579 & -0.66442 \\
$\mathrm{H}$ & -1.20538 & 4.79862 & -0.17051 \\
$\mathrm{H}$ & -2.33646 & 4.57929 & 1.17983 \\
$\mathrm{H}$ & -3.91386 & 3.42877 & -0.20946 \\
$\mathrm{H}$ & -3.01286 & 4.10482 & -1.58143 \\
$\mathrm{H}$ & 1.79149 & 4.65328 & -0.46055 \\
$\mathrm{H}$ & 2.92143 & 4.1014 & 1.5721 \\
$\mathrm{H}$ & 4.11402 & 3.11108 & 0.70883 \\
$\mathrm{H}$ & 3.20333 & 4.00101 & -1.31358 \\
$\mathrm{H}$ & 5.58696 & -0.50276 & 0.68932 \\
$\mathrm{H}$ & 5.70392 & -0.69953 & -1.07021 \\
$\mathrm{H}$ & 4.90887 & -2.95447 & -0.9586 \\
$\mathrm{H}$ & 5.24547 & -2.86911 & 0.78123 \\
$\mathrm{H}$ & -5.04708 & -2.83452 & 0.73207 \\
$\mathrm{H}$ & -5.50282 & -2.34069 & -0.90974 \\
$\mathrm{H}$ & -5.68877 & -0.0448 & -0.26293 \\
$\mathrm{H}$ & -5.68574 & -0.62958 & 1.41215 \\
& & & \\
\hline
\end{tabular}




\section{REFERENCES}

S1. Becke, A. D. Density-Functional thermochemistry.III. The Role of Exact Exchange. $J$. Chem. Phys. 1993, 98, 5648-5652.

S2. Lee, C.; Yang, W.; Parr, R. G. Development of the Colle-Salvetti Correlation-Energy Formula into a Functional of the Electron Density. Phys. Rev. B 1988, 37, 785-789.

S3. Dunning Jr, T. H. Gaussian Basis Sets for Use in Correlated Molecular Calculations. I. The Atoms Boron through Neon and Hydrogen. J. Chem. Phys. 1989, 90, 1007-1023.

S4. Dunning, J. T. H.; Woon, D. E. Gaussian Basis Sets for Use in Correlated Molecular Calculations. III. The Atoms Aluminum through Argon. J. Chem. Phys. 1993, 98, 13581371.

S5. Peterson, K. A.; Figgen, D.; Goll, E.; Stoll, H.; Dolg, M. Systematically Convergent Basis Sets with Relativistic Pseudopotentials. II. Small-Core Pseudopotentials and Correlation Consistent Basis Sets for the Post-D Group 16-18 Elements. J. Chem. Phys. 2003, 119, 11113-11123.

S6. Feller, D. The Role of Databases in Support of Computational Chemistry Calculations. $J$. Comput. Chem. 1996, 17, 1571-1586.

S7. Schuchardt, K. L.; Didier, B. T.; Elsethagen, T.; Sun, L.; Gurumoorthi, V.; Chase, J.; Li, J.; Windus, T. L. Basis Set Exchange: A Community Database for Computational Sciences. J. Chem. Inf. Model. 2007, 47, 1045-1052.

S8. Braun, C. A.; Zomerman, D.; de Aguiar, I.; Qi, Y.; Delgado, W. T.; Ferguson, M. J.; McDonald, R.; de Souza, G. L. C.; He, G.; Brown, A.; Rivard, E. Probing the Nature of Peripheral Boryl Groups within Luminescent Tellurophenes. Faraday Discuss. 2017, 196, 255-268.

S9. Peach, M. J. G.; Benfield, P.; Helgaker, T.; Tozer, D. J. Excitation Energies in Density Functional Theory: An Evaluation and a Diagnostic Test. J. Chem. Phys. 2008, 128, 44118.

S10. Zhao, Y.; Truhlar, D. G. The M06 Suite of Density Functionals for Main Group Thermochemistry, Thermochemical Kinetics, Noncovalent Interactions, Excited States, and Transition Elements: Two New Functionals and Systematic Testing of Four M06Class Functionals and 12 Other Function. Theor. Chem. Acc. 2008, 120, 215-241.

S11. Yanai, T.; Tew, D. P.; Handy, N. C. A New Hybrid Exchange-Correlation Functional Using the Coulomb-Attenuating Method (CAM-B3LYP). Chem. Phys. Lett. 2004, 393, $51-57$.

S12. Allouche, A.-R. Gabedit - A Graphical User Interface for Computational Chemistry Softwares. J. Comput. Chem. 2010, 32, 174-182. 
S13. Cancès, E.; Mennucci, B.; Tomasi, J. A New Integral Equation Formalism for the Polarizable Continuum Model: Theoretical Background and Applications to Isotropic and Anisotropic Dielectrics. J. Chem. Phys. 1997, 107, 3032-3041.

S14. Cossi, M.; Scalmani, G.; Rega, N.; Barone, V. New Developments in the Polarizable Continuum Model for Quantum Mechanical and Classical Calculations on Molecules in Solution. J. Chem. Phys. 2002, 117, 43-54.

S15. Gaussian 09, Revision D.01, Frisch, M. J.; Trucks, G. W.; Schlegel, H. B.; Scuseria, G. E.; Robb, M. A.; Cheeseman, J. R.; Scalmani, G.; Barone, V.; Mennucci, B.; Petersson, G. A.; Nakatsuji, H.; Caricato, M.; Li, X.; Hratchian, H. P.; Izmaylov, A. F.; Bloino, J.; Zheng, G.; Sonnenberg, J. L.; Hada, M.; Ehara, M.; Toyota, K.; Fukuda, R.; Hasegawa, J.; Ishida, M.; Nakajima, T.; Honda, Y.; Kitao, O.; Nakai, H.; Vreven, T.; Montgomery Jr., J. A.; Peralta, J. E.; Ogliaro, F.; Bearpark, M.; Heyd, J. J.; Brothers, E.; Kudin, K. N.; Staroverov, V. N.; Kobayashi, R.; Normand, J.; Raghavachari, K.; Rendell, A.; Burant, J. C.; Iyengar, S. S.; Tomasi, J.; Cossi, M.; Rega, N.; Millam, J. M.; Klene, M.; Knox, J. E.; Cross, J. B.; Bakken, V.; Adamo, C.; Jaramillo, J.; Gomperts, R.; Stratmann, R. E.; Yazyev, O.; Austin, A. J.; Cammi, R.; Pomelli, C.; Ochterski, J. W.; Martin, R. L.; Morokuma, K.; Zakrzewski, V. G.; Voth, G. A.; Salvador, P.; Dannenberg, J. J.; Dapprich, S.; Daniels, A. D.; Farkas, Ö.; Foresman, J. B.; Ortiz, J. V.; Cioslowski, J.; Fox, D. J. Gaussian, Inc., Wallingford CT (2009).

S16. He, G; Wiltshire, B.D.; Choi, P.; Savin, A.; Sun, S.; Mohammadpour, A.; Ferguson, M.J.; McDonald, R.; Brown, A.; Shankar, K.; and Rivard, E. Phosphorescence within benzotellurophenes and color tunable tellurophenes under ambient conditions. Chem Comm. 2015, 51, 5444-5447. 